\title{
Modelos aditivos generalizados com defasagens distribuídas
}

\author{
Alberto Pereira de Barros
}

\author{
Dissertação apresentada ao \\ Instituto de Matemática e Estatística \\ da Universidade de São Paulo \\ para obtenção do título de \\ Mestre em Estatística
}

\section{Área de Concentração: Estatística \\ Orientadora: Profa. Dra. Carmen Diva Saldiva de André}

Durante parte da elaboração deste trabalho o autor recebeu apoio financeiro do $\mathrm{CNPq}$

São Paulo, Julho de 2003 


\section{Modelos aditivos generalizados com defasagens distribuídas}

Este exemplar corresponde à redação final da dissertação devidamente corrigida e defendida por Alberto Pereira de Barros e aprovada pela comissão julgadora.

São Paulo, 02 de julho de 2003.

Comissão Julgadora:

- Profa. Dra. Carmen Diva Saldiva de André (orientadora) - IME/USP

- Profa. Dra. Lisbeth Kaiserlian Cordani - IME/USP

- Prof. Dr. Samuel Hazzan - IMQ/FGV 
Somente seja forte e muito corajoso! Tenha o cuidado de obedecer a toda a lei que o meu servo Moisés lhe ordenou; não se desvie dela, nem para a direita nem para a esquerda, para que você seja bem-sucedido por onde quer que andar. Não deixe de falar as palavras deste Livro da Lei e de meditar nelas de dia e de noite, para que você cumpra fielmente tudo o que nele está escrito. Só então os seus caminhos prosperarão e você será bem-sucedido. 


\section{Agradecimentos}

Este trabalho foi um grande desafio em minha vida, por diversas vezes achei que não iria conseguir concluí-lo. Por isso, não posso deixar de agradecer a algumas pessoas que direta ou indiretamente contribuíram para o sucesso dele.

Primeiramente, gostaria de agradecer a Deus, meu Pai Celestial, por me amar tanto e continuar me amando mesmo quando não me importo com as coisas que Ele me diz. Obrigado por ter preparado todo o caminho, desde quando ainda estava na barriga da minha mãe até a conclusão do Mestrado, embora devo reconhecer que em muitos momentos não confiei que o Senhor estava cuidando de tudo.

Gostaria de agradecer à Professora Carmen por sua orientação, humildade, paciência e por dividir comigo todo o seu conhecimento a respeito dos modelos aditivos generalizados. Sem dúvida, sua presença ao meu lado foi de extrema importância na realização deste trabalho.

Agradeço também à minha família, especialmente aos meus pais, por tudo o que me ensinaram e contribuíram para a formação do meu caráter. Obrigado pela torcida e pelo apoio financeiro.

Agradeço à USP e aos professores do IME, por tudo o que me ensinaram, e ao CNPq cujo auxílio financeiro tornou possível a realização deste trabalho.

Quero agradecer também a todos amigos do IME, sobretudo aos usuários da sala 250. Obrigado pela amizade e torcida de vocês. Em especial, gostaria de agradecer à Adrilayne, que sempre me socorreu nos momentos mais difíceis deste trabalho, além de ter sido minha grande companheira e apoiadora nas diversas listas de exercícios que fazíamos juntos nos finais de semana e feriados prolongados.

Por fim, gostaria de agradecer a todos os meus irmãos em Cristo que sempre me ajudaram a olhar para Deus nos momentos mais desafiantes deste trabalho, especialmente ao Kazuo e ao Paulinho pela extrema servidão, cuidando da limpeza 
da casa, cozinhando e guardando um prato de comida para que eu pudesse me alimentar quando chegava tarde em casa, após passar longas horas no IME. Todos que fazem parte da minha família espiritual são importantes para mim, obrigado pela amizade e o apoio de cada um, mas não posso deixar de agradecer à algumas pessoas muito próximas que estão sempre me amando e me ajudando a estar mais perto de Deus, entre elas ao Ronaldo, Emerson, Felipe, Diniz e Marcos. Obrigado pela amizade e lealdade que sempre demonstraram por mim. O amor e compaixão que vocês têm pelas pessoas só me inspiram a lutar para estar mais próximo de Deus. E, como dizem as Escrituras, o sucesso e a prosperidade não estão nos bens materiais que possamos adquirir, mas sim em seguir o caminho traçado por Deus para as nossas vidas. 


\section{Resumo}

Existem algumas situações onde a variável resposta é observada repetidamente ao longo do tempo, e o impacto de uma variável preditora em um determinado instante é distribuído sobre várias medidas subsequentes da variável resposta. Por outro lado, outras variáveis preditoras no modelo podem se relacionar com a resposta de forma não linear. Para construir um modelo de regressão adequado para esta situação combinamos duas técnicas: modelos aditivos generalizados e modelos com defasagens distribuidas. Os modelos aditivos generalizados são uma extensão dos modelos lineares generalizados no qual variáveis preditoras contínuas são modeladas por funções não especificadas. Já os modelos com defasagens distribuídas relacionam a variável resposta com valores defasados de uma variável preditora dependente do tempo. A combinação dessas duas técnicas de modelagem resulta nos chamados modelos aditivos generalizados com defasagens distribuidas. A estimação é feita pelo método de máxima verossimilhança penalizada de acordo com a suposição da distribuição da variável resposta. As estimativas são então obtidas combinando o método de mínimos quadrados reponderados iterativamente com a técnica de suavização $P$-spline. Esta metodologia é aplicada a dados ambientais da cidade de São Paulo para investigar a distribuição do efeito da poluição do ar sobre a natimortalidade e para quantificar o denominado efeito colheita. 


\begin{abstract}
There are several situations, where a response variable is observed repeatedly over time, and the impact of a predictor at one time is distributed over several subsequent response measures. On the other hand, other predictors in the model impact the response in a non-linear fashion. To construct a regression model suitable to this situation we combine two regression techniques: generalized additive models and distributed lag models. Generalized additive models are an extension of generalized linear models in which continuous predictors are modeled as unspecified functions. Distributed lag models aim to relate the response variable to lagged values of the time-dependent predictor. The combination of these two modeling techniques results in the so-called generalized additive distributed lag models. Estimation is performed by maximum penalized likelihood, according to the distributional assumption for the procedure combined with a smoothing technique called $P$-spline. This metodology is applied to environmental data from São Paulo city to investigate the distribution of the air pollution effect over time on the fetal death, and to quantify the so-called mortality displacement effect.
\end{abstract}


1. Introdução. 01

2. Modelos de regressão com defasagens distribuídas.....................................07

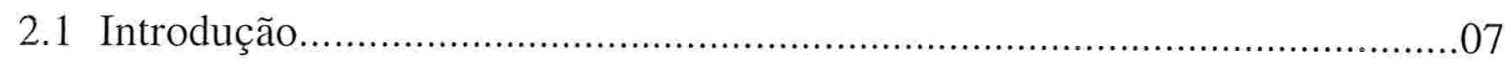

2.2 A escolha da defasagem máxima (q) e do grau do polinômio (d)................12

2.3 O teste da adequação da restrição polinomial..........................................13

2.4 Modelos aditivos generalizados com defasagens distribuídas....................15

3. Modelos com defasagens distribuídas com restrição não paramétrica.........24

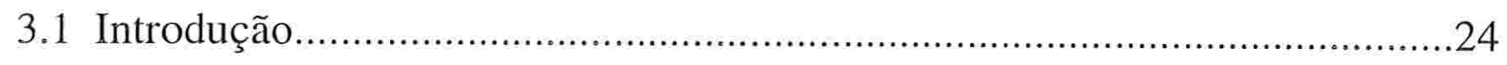

3.2. Regressão spline e o suavizador P-spline ..............................................25

3.3 Utilização do $P$-spline no ajuste de modelos com defasagens distribuídas........29

3.4 Utilização do $P$-spline na estimação de modelos aditivos generalizados com

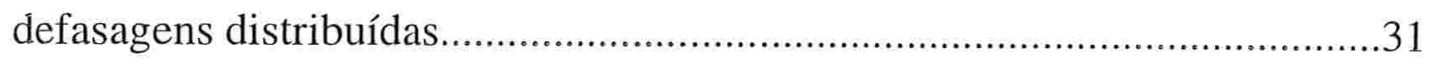

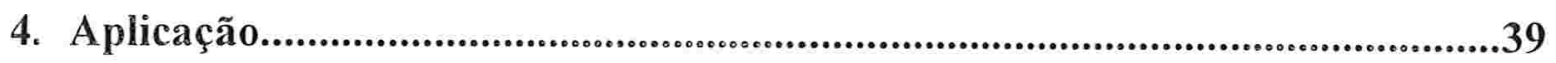

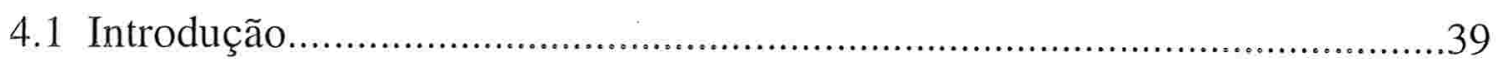

4.2 Ajuste de modelos aditivos generalizados com defasagens de até 4 dias entre o poluente e a natimortalidade ...........................................................4 42

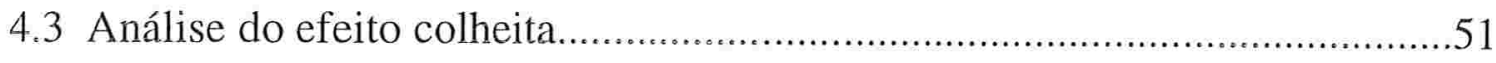

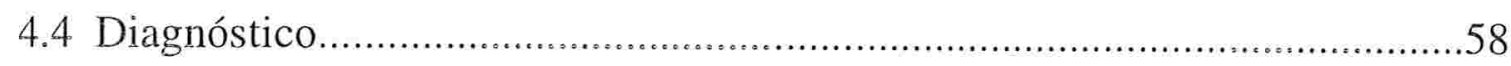

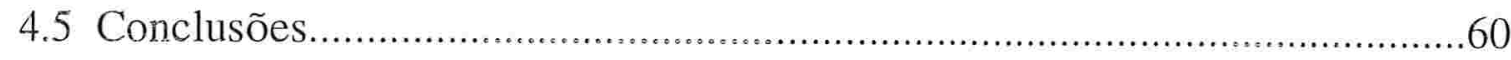




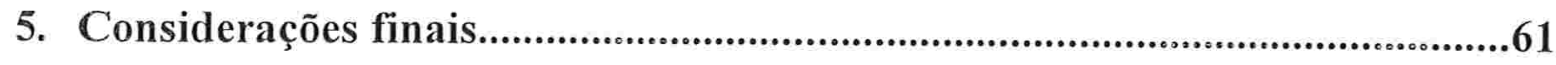

A: Alguns resultados de ajustes de modelos aditivos generalizados com defasagens de até 7 dias do poluente em relação ao evento de

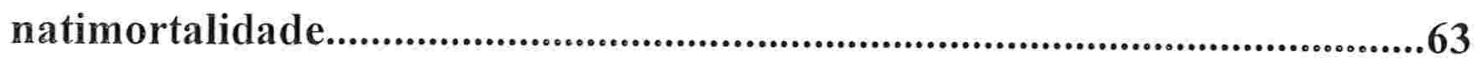

B: Resultados do ajuste do modelo (4.9).............................................................

C: Comandos do S-plus para a obtenção dos resultados do modelo (4.9)........ .73

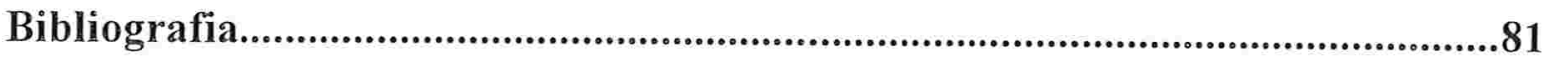




\section{Capítulo 1}

Introdução

Em um modelo de regressão pode ocorrer que variações nos valores observados da variável resposta não sejam observadas no mesmo instante em que ocorrem alterações nos valores das variáveis explicativas. Ao invés disso, o efeito das mudanças nas variáveis explicativas na resposta ocorre de uma maneira gradual ao longo do tempo. Diz-se então haver defasagem entre as variáveis explicativas e a variável resposta, o que levou à formulação dos modelos com defasagens distribuídas. Um exemplo de modelo nessa classe é

$$
Y_{t}=\alpha+\beta_{0} X_{t}+\beta_{1} X_{t-1}+\ldots+\beta_{q} X_{t-q}+\varepsilon_{t}, \quad t=q+1, \ldots, n,(1,1
$$

onde

$\left(\mathrm{Y}_{\mathrm{t}}, \mathrm{X}_{\mathrm{t}}\right)$ correspondem aos valores de uma variável resposta $\mathrm{Y}$ e uma variável não estocástica X em um instante de tempo t;

$\mathrm{X}_{\mathrm{t}-1}, \ldots, \mathrm{X}_{\mathrm{t}-\mathrm{q}}$ são os valores de $\mathrm{X}$ em $1, \ldots, \mathrm{q}$ unidades de tempo anteriores ao instante $\mathrm{t}$, respectivamente;

$\alpha, \beta_{0}, \beta_{1}, \ldots, \beta_{\mathrm{q}}$ são parâmetros a serem estimados e

$\varepsilon_{\mathrm{t}}$ é um erro aleatório tal que $\mathrm{E}\left(\varepsilon_{\mathrm{t}}\right)=0, \operatorname{var}\left(\varepsilon_{\mathrm{t}}\right)=\sigma^{2} \mathrm{e} \operatorname{cov}\left(\varepsilon_{\mathrm{s}}, \varepsilon_{\mathrm{t}}\right)=0 \forall \mathrm{s} \neq \mathrm{t}, \mathrm{t}=\mathrm{q}+1, \ldots, \mathrm{n}$.

Os primeiros trabalhos envolvendo esses modelos surgiram em problemas ligados à Economia em meados de 1930. Exemplos da sua aplicação nessa área podem ser encontrados, por exemplo, em Hazzan (1978) e Davidson e MacKinnon (1993). 
Recentemente, Pope e Schwartz (1996) propuseram a utilização de modelos com defasagens distribuídas para descrever a associação entre as concentrações de poluentes atmosféricos e o número de mortes ou internações hospitalares por causas respiratórias. Neste tipo de estudo é considerado que o número de mortes ou internações hospitalares ocorridas em um certo dia dependem do efeito dos níveis de concentração dos poluentes neste mesmo dia, no dia anterior, etc, ou seja, existe defasagem entre o nível de concentração dos poluentes e o número de mortes ou internações hospitalares ocorridas em um determinado dia. Segundo Schwartz (2000a), o efeito da poluição do ar na população em períodos de defasagem curtos é distribuído no tempo como ilustrado na Figura 1.1.

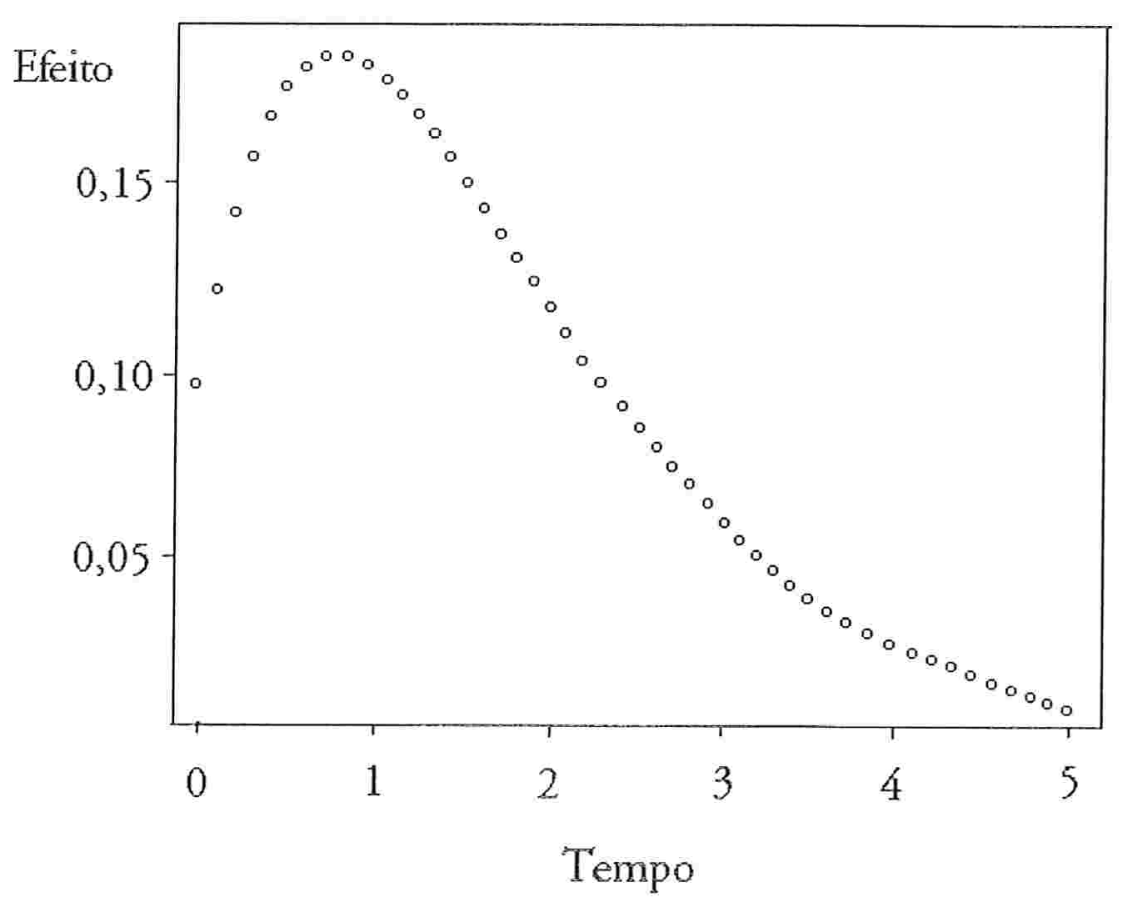

Figura 1.1: Efeito da poluição do ar na população em períodos curtos de defasagem. 
Conforme foi considerado, entre outros, por Zanobetti et al.(2000), em um indivíduo o risco causado pela exposição à poluição do ar é presumivelmente maior ou igual a zero, qualquer que seja a defasagem. Já na população isto não necessariamente ocorre. Suponha que em um determinado instante de tempo exista um grupo de pessoas com elevado risco de morte devido às condições agudas ou crônicas de saúde. Então um episódio de poluição do ar pode aumentar o risco de mortalidade desses indivíduos e antecipar sua morte, fazendo com que nos dias subsequentes o número de mortes observados seja menor que o esperado e ocorra associação negativa entre mortalidade (ou morbidade) e poluição do ar. A natureza finita deste grupo de risco cria a possibilidade desta associação negativa, pois muitas das mortes observadas durante o episódio de alta concentração dos poluentes foram antecipadas por um curto período de tempo. A este fenômeno dá-se o nome de efeito colheita. No estudo da ocorrência desse efeito, são considerados longos períodos de defasagem, em geral de 30 a 45 dias.

A Figura 1.2 ilustra o tipo de comportamento que é esperado dos coeficientes do poluente em um modelo de regressão quando existe efeito colheita. No eixo horizontal está representada a defasagem em $\mathrm{q}=30$ unidades de tempo anteriores ao instante de tempo t em que foi observado o evento de mortalidade ou morbidade, e no vertical os coeficientes das concentrações do poluente nas diferentes defasagens consideradas. A área A é aproximadamente igual à soma dos coeficientes positivos das concentrações defasadas de curto período de tempo e que têm efeito positivo na variável resposta. Os coeficientes representados em B são negativos o que significa 
que as concentrações do poluente, em um período de tempo mais distante, têm associação negativa com a mortalidade ou morbidade. Este fato ocorre devido à antecipação da morte das pessoas que constituem o grupo de risco, formado principalmente por idosos e crianças (Singer et al., 2002), por um episódio de alta concentração da poluição do ar. Se existe um recrutamento de indivíduos para recompor o grupo de risco, o que pode ocorrer de forma mais lenta do que o acréscimo da mortalidade em A, é de se esperar, após o período B, a ocorrência de um período C no qual os coeficientes da regressão voltam a ser positivos. Descrições mais detalhadas do efeito colheita podem ser encontradas em Zanobetti et al.(2000) e Schwartz (2000b).

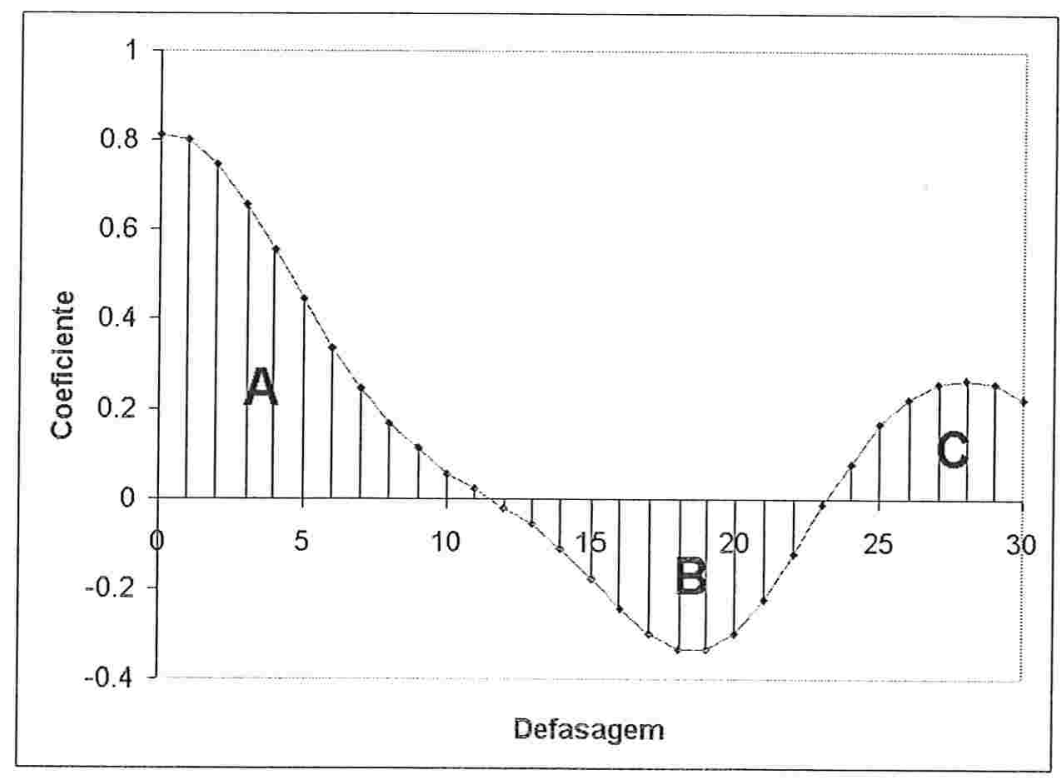

Figura 1.2: Estrutura de defasagem esperada na ocorrência do efeito colheita 
Nosso objetivo neste trabalho é apresentar modelos de regressão com defasagens distribuídas adequados para investigar a distribuição do efeito da poluição atmosférica na mortalidade ou na morbidade da população a curto e longo prazos.

O trabalho foi organizado da seguinte forma: no Capítulo 2 descrevemos os modelos de regressão com defasagens distribuídas, considerando inicialmente modelos lineares. Duas versões do modelo são apresentadas: sem e com a restrição nos parâmetros que é imposta com o objetivo de minimizar os problemas ocasionados pela existência de multicolinearidade entre as variáveis defasadas. Alguns resultados sobre modelos aditivos generalizados (Hastie e Tibishirani, 1990) são também apresentados nesse capítulo, sendo considerada uma versão desse modelo que inclui variáveis explicativas defasadas em relação à resposta, devido à freqüente utilização desses modelos na área de aplicação de interesse neste trabalho. No Capítulo 3 apresentamos os métodos de suavização regressão spline e P-spline (Eilers e Marx, 1996 ou Marx e Eilers, 1998) com o objetivo de utilizá-los no ajuste de modelos aditivos generalizados com defasagens distribuídas com restrição nos parâmetros, quando longos períodos de defasagem são considerados, isto é, no estudo do efeito colheita. Mostramos nesse capítulo que a adoção desses métodos de suavização reduz o custo computacional envolvido no ajuste dos modelos aditivos generalizados em relação a outros métodos. Nos modelos com restrição, é possível, com esses métodos descrever de forma mais flexível o comportamento dos coeficientes da variável defasada em função do tempo. Como ilustração, no Capítulo 4, adotamos modelos aditivos generalizados com defasagens distribuídas na reanálise dos dados obtidos por Pereira et al. (1998) com a 
finalidade de investigar o efeito do poluente $\mathrm{NO}_{2}$ na mortalidade fetal tardia na cidade de São Paulo, durante os anos de 1991 e 1992. Foram considerados longos e curtos períodos de defasagem entre a natimortalidade e as concentrações dos poluentes e modelos com e sem restrição nos parâmetros. Algumas considerações finais sobre o trabalho e propostas para estudos futuros são feitas no capítulo 5 . 
Capítulo 2

\section{Modelos de regressão com defasagens distribuídas}

\subsection{Introdução}

O modelo linear com defasagens distribuídas tem a forma

$$
Y_{t}=\alpha+\beta_{0} X_{t}+\beta_{1} X_{t-1}+\ldots+\beta_{q} X_{t-q}+\varepsilon_{t}, \quad t=q+1, \ldots, n,(2.1)
$$

onde

$\left(Y_{t}, X_{t}\right)$ correspondem aos valores de uma variável resposta $Y$ e uma variável não estocástica X em um instante de tempo t;

$X_{t-1}, \ldots, X_{t-q}$ são os valores de $X$ em $1, \ldots, q$ unidades de tempo anteriores ao instante $\mathfrak{t}$, respectivamente;

$\alpha, \beta_{0}, \beta_{1}, \ldots, \beta_{\mathrm{q}}$ são parâmetros a serem estimados e

$\varepsilon_{\mathrm{t}}$ é um erro aleatório tal que $\mathrm{E}\left(\varepsilon_{\mathrm{t}}\right)=0, \operatorname{var}\left(\varepsilon_{\mathrm{t}}\right)=\sigma^{2} \mathrm{e} \operatorname{cov}\left(\varepsilon_{\mathrm{s}}, \varepsilon_{\mathrm{t}}\right)=0 \forall \mathrm{s} \neq \mathrm{t}, \mathrm{t}=\mathrm{q}+1, \ldots, \mathrm{n}$.

Assim, $\beta_{0}, \beta_{1}, \ldots, \beta_{\mathrm{q}}$ são os coeficientes da variável preditora $X$, defasada de $0,1, \ldots, q$ unidades de tempo em relação ao instante $\mathrm{t}$.

A equação (2.1) pode ser reescrita na forma:

$$
\mathrm{Y}=\mathbf{X} \boldsymbol{\beta}+\varepsilon
$$

onde $\mathrm{Y}=\left(\begin{array}{llll}\mathrm{y}_{\mathrm{q}+1} & \mathrm{y}_{\mathrm{q}+2} & \cdots & \mathrm{y}_{\mathrm{n}}\end{array}\right)^{\mathrm{T}}$,

$$
X=\left[\begin{array}{ccccc}
1 & x_{q+1} & x_{q} & \cdots & x_{1} \\
1 & x_{q+2} & x_{q+1} & \cdots & x_{2} \\
\vdots & \vdots & \vdots & \ddots & \vdots \\
1 & x_{n} & x_{n-1} & \cdots & x_{n-q}
\end{array}\right]
$$




$$
\begin{aligned}
& \boldsymbol{\beta}=\left(\begin{array}{llll}
\alpha & \beta_{0} & \cdots & \beta_{\mathrm{q}}
\end{array}\right)^{\mathrm{T}} \mathrm{e} \\
& \boldsymbol{\varepsilon}=\left(\begin{array}{lllll}
\varepsilon_{\mathrm{q}+1} & \varepsilon_{\mathrm{q}+2} & \cdots & \varepsilon_{\mathrm{n}}
\end{array}\right)^{\mathrm{T}} .
\end{aligned}
$$

Os parâmetros do modelo (2.1) poderiam, a princípio, ser estimados pelo método de mínimos quadrados. Entretanto, as variáveis preditoras $X_{t}, X_{t-1}, \ldots, X_{t-q}$ são, em geral, altamente correlacionadas, e as consequências do problema de multicolinearidade podem afetar as estimativas obtidas, tornando-as bastante imprecisas, isto é, com erros padrão estimados elevados. Este problema afeta os testes de significância sobre os coeficientes do modelo. Uma descrição completa sobre os problemas causados pela multicolinearidade no ajuste de modelos de regressão podem ser encontrados, entre outros, em Neter et al., (1996).

Uma forma de superar os problemas causados pela multicolinearidade é impor restrições aos coeficientes do modelo de forma a reduzir o número de parâmetros a serem estimados e, consequentemente, o número de variáveis preditoras no modelo. Com este objetivo, o modelo proposto por Almon (1965) admite que $\beta_{0}, \beta_{1}, \ldots, \beta_{\mathrm{q}}$ são valores de uma função definida em um intervalo fechado e, portanto, pode ser aproximada em todo o seu domínio por uma função polinomial de grau conveniente, conforme ilustrado na Figura 2.1.

Além do modelo de Almon, existem outros obtidos a partir de outras restrições nos coeficientes e podem ser vistos em Hazzan (1978). 


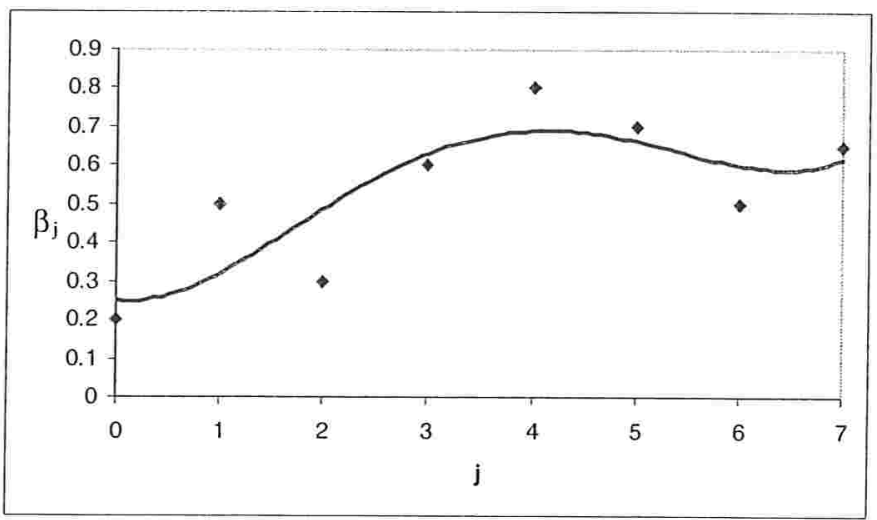

Figura 2.1: Aproximação dos coeficientes do modelo por uma função polinomial

Como a estimação dos coeficientes a partir de tais restrições envolve procedimentos de alto custo computacional (Hazzan, 1978), uma modificação bastante utilizada consiste em admitir diretamente que os parâmetros $\beta_{0}, \beta_{1}, \ldots, \beta_{\mathrm{q}}$ são valores de uma função polinomial de grau d $(\mathrm{d}<\mathrm{q})$. São, então, impostas aos coeficientes do modelo (2.1) as seguintes restrições:

$$
\beta_{j}=f(j)=\sum_{k=0}^{d} \tau_{k} j^{k} \quad j=0, \ldots, q,
$$

isto é,

$$
\begin{aligned}
& \beta_{0}=\mathrm{f}(0)=\tau_{0} \\
& \beta_{1}=\mathrm{f}(1)=\tau_{0}+\tau_{1}+\tau_{2}+\ldots+\tau_{\mathrm{d}} \\
& \beta_{2}=\mathrm{f}(2)=\tau_{0}+2 \tau_{1}+2^{2} \tau_{2}+\ldots+2^{\mathrm{d}} \tau_{\mathrm{d}} \\
& \vdots \\
& \beta_{\mathrm{q}}=\mathrm{f}(\mathrm{q})=\tau_{0}+\mathrm{q} \tau_{1}+\mathrm{q}^{2} \tau_{2}+\ldots+\mathrm{q}^{\mathrm{d}} \tau_{\mathrm{d}},
\end{aligned}
$$

ou seja,

$$
\beta=C \tau,
$$


onde

$$
\mathrm{C}_{(\mathrm{q}+2) \times(\mathrm{d}+2)}=\left[\begin{array}{cccccc}
1 & 0 & 0 & 0 & \cdots & 0 \\
0 & 1 & 0 & 0^{2} & \cdots & 0^{\mathrm{d}} \\
0 & 1 & 1 & 1^{2} & \cdots & 1^{\mathrm{d}} \\
0 & 1 & 2 & 2^{2} & \cdots & 2^{\mathrm{d}} \\
\vdots & \vdots & \vdots & \vdots & \ddots & \vdots \\
0 & 1 & \mathrm{q} & \mathrm{q}^{2} & \cdots & \mathrm{q}^{\mathrm{d}}
\end{array}\right]
$$

e $\tau=\left(\begin{array}{lllll}\alpha & \tau_{0} & \tau_{1} & \cdots & \tau_{\mathrm{d}}\end{array}\right)^{\mathrm{T}}$ é um vetor de parâmetros desconhecidos de dimensão $(\mathrm{d}+2)$ menor que a de $\boldsymbol{\beta}$.

Substituindo as equações (2.4) no modelo (2.1) temos:

$$
\begin{aligned}
\mathrm{Y}_{\mathrm{t}}= & \alpha+\tau_{0} \mathrm{X}_{\mathrm{t}}+\left(\tau_{0}+\tau_{1}+\tau_{2}+\ldots+\tau_{\mathrm{d}}\right) \mathrm{X}_{\mathrm{t}-1}+\left(\tau_{0}+2 \tau_{1}+2^{2} \tau_{2}+\ldots+2^{\mathrm{d}} \tau_{\mathrm{d}}\right) \mathrm{X}_{\mathrm{t}-2} \\
& +\ldots+\left(\tau_{0}+\mathrm{q} \tau_{1}+\mathrm{q}^{2} \tau_{2}+\ldots+\mathrm{q}^{\mathrm{d}} \tau_{\mathrm{d}}\right) \mathrm{X}_{\mathrm{t}-\mathrm{q}}+\varepsilon_{\mathrm{t}} \\
= & \alpha+\tau_{0}\left(\mathrm{X}_{\mathrm{t}}+\mathrm{X}_{\mathrm{t}-1}+\mathrm{X}_{\mathrm{t}-2}+\ldots+\mathrm{X}_{\mathrm{t}-\mathrm{q}}\right)+\tau_{\mathrm{l}}\left(\mathrm{X}_{\mathrm{t}-1}+2 \mathrm{X}_{\mathrm{t}-2}+\ldots+\mathrm{q} \mathrm{X}_{\mathrm{t}-\mathrm{q}}\right)+ \\
& \tau_{2}\left(\mathrm{X}_{\mathrm{t}-1}+2^{2} \mathrm{X}_{\mathrm{t}-2}+\ldots+\mathrm{q}^{2} \mathrm{X}_{\mathrm{t}-\mathrm{q}}\right)+\ldots+\tau_{\mathrm{d}}\left(\mathrm{X}_{\mathrm{t}-1}+2^{\mathrm{d}} \mathrm{X}_{\mathrm{t}-2}+\ldots+\mathrm{q}^{\mathrm{d}} \mathrm{X}_{\mathrm{t}-\mathrm{q}}\right)+\varepsilon_{\mathrm{t}} \\
= & \alpha+\tau_{0} \sum_{\mathrm{j}=0}^{\mathrm{q}} \mathrm{X}_{\mathrm{t}-\mathrm{j}}+\tau_{1} \sum_{\mathrm{j}=0}^{\mathrm{q}} \mathrm{j} \mathrm{X}_{\mathrm{t}-\mathrm{j}}+\tau_{2} \sum_{\mathrm{j}=0}^{\mathrm{q}} \mathrm{j}^{2} \mathrm{X}_{\mathrm{t}-\mathrm{j}}+\ldots+\tau_{\mathrm{d}} \sum_{\mathrm{j}=0}^{\mathrm{q}} \mathrm{j}^{\mathrm{d}} \mathrm{X}_{\mathrm{t}-\mathrm{j}}+\varepsilon_{\mathrm{t}} \\
= & \alpha+\sum_{\mathrm{k}=0}^{\mathrm{d}} \tau_{\mathrm{k}}\left(\sum_{\mathrm{j}=0}^{\mathrm{q}} \mathrm{j}^{\mathrm{k}} \mathrm{X}_{\mathrm{t}-\mathrm{j}}\right)=\alpha+\sum_{\mathrm{k}=0}^{\mathrm{d}} \tau_{\mathrm{k}} \mathrm{Z}_{\mathrm{tk}}
\end{aligned}
$$

onde,

$$
Z_{t k}=\sum_{j=0}^{\mathrm{q}} \mathrm{j}^{\mathrm{k}} \mathrm{X}_{\mathrm{t}-\mathrm{j}}, \quad \mathrm{t}=\mathrm{q}+1, \mathrm{q}+2, \ldots, \mathrm{n}, \mathrm{k}=0,1,2, \ldots, \mathrm{d} .
$$

Assim, o modelo (2.1) pode ser escrito como:

$$
Y_{t}=\alpha+\tau_{0} Z_{t 0}+\tau_{1} Z_{t 1}+\tau_{2} Z_{t 2}+\ldots+\tau_{d} Z_{t d}+\varepsilon_{t}, \quad t=q+1, q+2, \ldots, n
$$

A equação (2.8) caracteriza um modelo com defasagens distribuídas com restrição, ou modelo polinomial de Âlmon. Essa equação pode também ser escrita como: 


$$
\mathbf{Y}=\mathbb{Z} \tau+\varepsilon
$$

onde

Y e $\varepsilon$ são definidos como em (2.2),

$\tau=\left(\begin{array}{lllll}\alpha & \tau_{0} & \tau_{1} \cdots & \tau_{\mathrm{d}}\end{array}\right)^{\mathrm{T}}$,

e $\mathbf{Z}$ é uma matriz de dimensão $(n-q) x(d+2)$ dada por

$$
Z=\left[\begin{array}{ccccc}
1 & \mathrm{Z}_{(\mathrm{q}+1) 0} & \mathrm{Z}_{(\mathrm{q}+1) 1} & \cdots & \mathrm{Z}_{(\mathrm{q}+1) \mathrm{d}} \\
1 & \mathrm{Z}_{(\mathrm{q}+2) 0} & \mathrm{Z}_{(\mathrm{q}+2) 1} & \cdots & \mathrm{Z}_{(\mathrm{q}+2) \mathrm{d}} \\
\vdots & \vdots & \vdots & \ddots & \vdots \\
1 & \mathrm{Z}_{\mathrm{n} 0} & \mathrm{Z}_{\mathrm{n} 1} & \cdots & \mathrm{Z}_{\mathrm{nd}}
\end{array}\right]
$$

cujos elementos são definidos em (2.7).

Devido à suposição sobre os erros do modelo (2.1), podemos afirmar que o estimador de mínimos quadrados $\hat{\tau}$ do vetor de parâmetros $\tau$ é dado por

$$
\hat{\tau}=\left(Z^{\mathrm{T}} \mathbf{Z}\right)^{-1} \mathbf{Z}^{\mathrm{T}} \mathbf{Y} .
$$

Além disso, assumindo que $\boldsymbol{\varepsilon} \sim \mathrm{N}\left(\boldsymbol{0}, \sigma^{2} \mathbf{I}\right)$, temos

$$
\hat{\boldsymbol{\tau}} \sim \mathrm{N}\left[\tau, \sigma^{2}\left(\mathbf{Z}^{\mathrm{T}} \mathbf{Z}\right)^{-1}\right],
$$

e consequentemente

$$
\hat{\boldsymbol{\beta}} \sim \mathrm{N}\left[\boldsymbol{\beta}, \sigma^{2} \mathbf{C}\left(\mathbf{Z}^{\mathrm{T}} \mathbf{Z}\right)^{-1} \mathbf{C}^{\mathrm{T}}\right] .
$$

Um estimador para $\sigma^{2}$ é dado por

$$
\hat{\sigma}^{2}=\frac{1}{n-q-d-2} Y^{\mathrm{T}}\left[\mathbf{I}-\underline{Z}\left(Z^{\mathrm{T}} \mathbf{Z}\right)^{-1} \mathbf{Z}^{\mathrm{T}}\right] \mathbf{Y} .
$$

Tem-se ainda que

$$
(n-q-d-2) \frac{\hat{\sigma}^{2}}{\sigma^{2}} \sim \chi_{n-q-d-2}^{2} \text {. }
$$




\subsection{A escolha da defasagem máxima (q) e do grau do polinômio (d)}

Existem várias alternativas para se escolher a defasagem máxima (q) e o grau do polinômio (d) (ver, por exemplo, Davidson e MacKinnon, 1993).

Para escolher q nos modelos com ou sem restrição, uma das alternativas mais utilizadas foi proposta por Almon(1965), e consiste em ajustarem-se modelos com diferentes valores para q e verificar qual deles apresenta maior valor do coeficiente de determinação múltipla corrigido pelo número de graus de liberdade, dado por

$$
R_{\text {aj }}^{2}=1-\frac{S Q R /(n-q-d-2)}{S Q T /(n-q-1)}=1-\frac{(n-q-1)}{(n-q-d-2)} \frac{S Q R}{S Q T}
$$

onde SQR é a soma de quadrados dos resíduos dada por

$$
\mathrm{SQR}=\left\{\begin{array}{cc}
\mathbf{Y}^{\mathrm{T}} \mathbf{Y}-\hat{\boldsymbol{\beta}} \mathbf{X}^{\mathrm{T}} \mathbf{Y}, & \text { para o modelo sem restrição (2.1) } \\
\mathbf{Y}^{\mathrm{T}} \mathbf{Y}-\hat{\boldsymbol{\tau}} \mathbf{Z}^{\mathrm{T}} \mathbf{Y}, & \text { para o modelo com restrição (2.8) }
\end{array},\right.
$$

$\operatorname{com} \hat{\boldsymbol{\beta}}=\left(\mathbf{X}^{\mathrm{T}} \mathbf{X}\right)^{-1} \mathbf{X}^{\mathrm{T}} \mathbf{Y}$ e $\hat{\boldsymbol{\tau}}$ como em (2.11), e SQT é a soma de quadrados total dada por

$$
\mathrm{SQT}=\mathrm{Y}^{\mathrm{T}} \mathrm{Y}-\frac{1}{\mathrm{n}-\mathrm{q}} \mathbf{Y}^{\mathrm{T}} \mathbf{1} 1^{\mathrm{T}} \mathbf{Y}
$$

Nos modelos com restrição, a escolha do grau do polinômio pode ser feita por meio de testes de significância sobre os coeficientes da equação (2.8). Considerando um valor para d fixado inicialmente, o que se faz é testar sequencialmente as hipóteses $\mathrm{H}_{01}: \tau_{\mathrm{d}}=0, \mathrm{H}_{02}: \tau_{\mathrm{d}-1}=0$, etc, até se encontrar evidências para a rejeição.

Por exemplo, se for fixado $d=4$, testa-se inicialmente $\mathrm{H}_{01}: \tau_{4}=0$ e, se esta hipótese for rejeitada, adota-se $d=4$ como grau do polinômio. Caso contrário, será 
testada a hipótese $\mathrm{H}_{02}: \tau_{3}=0$, e procede-se desta forma até ser encontrado algum $\tau_{\mathrm{k}}$ significantemente diferente de zero e adotando este k como o grau do polinômio.

A estatística usada para testar $\mathrm{H}_{0}: \tau_{\mathrm{k}}=0$ contra $\mathrm{H}_{1}: \tau_{\mathrm{k}} \neq 0, \mathrm{k}=\mathrm{d}, \mathrm{d}-1, \ldots, 0$, é dada por:

$$
\mathrm{F}=\frac{\mathrm{SQ}_{\mathrm{Reg}}}{1} \div \frac{\mathrm{SQR}}{\mathrm{n}-\mathrm{q}-\mathrm{d}-2}=(\mathrm{n}-\mathrm{q}-\mathrm{d}-2) \frac{\mathrm{SQ}_{\mathrm{Reg}}}{\mathrm{SQR}}
$$

onde $S Q_{\text {Reg }}$ é a soma de quadrados devido ao modelo dada por:

$$
S Q_{\text {Reg }}=\left(\mathbf{Z}^{* \mathrm{~T}} \mathbf{Z}^{*}\right)^{-1} \mathbf{Z}^{* \mathrm{~T}} \mathbf{Y} \mathbf{Z}^{* \mathrm{~T}} \mathbf{Y}-\left(\mathbf{Z}^{\mathrm{T}} \mathbf{Z}\right)^{-1} \mathbf{Z}^{\mathrm{T}} \mathbf{Y} \mathbf{Z}^{\mathrm{T}} \mathbf{Y}
$$

onde $\mathbb{Z}^{*}$ é a matriz definida em (2.10) sob a hipótese, $\mathbf{Z}$ é como em (2.10) e SQR é a soma de quadrados residual do modelo com restrição dada em (2.17).

Na escolha para q e d, entretanto, o que deve prevalecer é o bom senso do pesquisador. Em muitos casos, embora estatisticamente as técnicas apontem para um determinado valor de $\mathrm{q}$ ou $\mathrm{d}$, do ponto de vista da área de pesquisa na qual os dados foram coletados esta escolha não faz sentido algum. Portanto a utilização destas técnicas são válidas quando não temos nenhuma idéia a priori de qual deve ser o valor de q e d (Davidson e MacKinnon, 1993).

\subsection{0 teste da adequação da restrição polinomial}

Para avaliar se o ajuste de um modelo com as restrições nos parâmetros dadas em (2.3) é mais adequado que o ajuste de um modelo sem essas restrições, pode-se testar a hipótese: 


$$
\mathrm{H}_{0}: \boldsymbol{\beta}=\mathbf{C} \tau,
$$

onde C é definida em (2.5), contra a hipótese alternativa,

$$
\mathrm{H}_{1}: \boldsymbol{\beta} \text { não obedece a essa restrição. }
$$

O teste é feito comparando-se, em uma escala adequada, as somas de quadrados residuais obtidas no ajuste dos dois modelos (Davidson e MacKinnon, 1993).

Sob o modelo com restrição (2.8), isto é, sob $\mathrm{H}_{0}$, a soma de quadrados dos resíduos é dada por

$$
\mathrm{SQR}_{0}=\mathrm{Y}^{\mathrm{T}}\left[\mathrm{I}-\mathbf{X} \mathbf{C}\left(\mathbf{C}^{\mathrm{T}} \mathbf{X}^{\mathrm{T}} \mathbf{X} \mathbf{C}\right)^{-1} \mathrm{C}^{\mathrm{T}} \mathbf{X}^{\mathrm{T}}\right] \mathrm{Y}
$$

No modelo irrestrito (2.1), ela é dada por

$$
\mathrm{SQR}_{1}=\mathbf{Y}^{\mathrm{T}}\left[\mathbf{I}-\mathbf{X}\left(\mathbf{X}^{\mathrm{T}} \mathbf{X}\right)^{-1} \mathbf{X}^{\mathrm{T}}\right] \mathbf{Y}
$$

Supondo nos dois modelos que $\varepsilon \sim \mathrm{N}\left(\mathbf{0}, \sigma^{2} \mathbf{I}\right)$ e considerando que as matrizes das formas quadráticas (2.21) e (2.22) são idempotentes de posto d-2 e q-2 respectivamente, temos que:

$$
\frac{\mathrm{SQR}_{0}}{\sigma^{2}} \sim \chi_{\mathrm{n}-\mathrm{q}-\mathrm{d}-2}^{2}, \frac{\mathrm{SQR}_{1}}{\sigma^{2}} \sim \chi_{\mathrm{n}-2 \mathrm{q}-2}^{2}
$$

Seja

$$
\mathrm{Q}=\mathrm{SQR}_{0}-\mathrm{SQR}_{1}=\mathbf{Y}^{\mathrm{T}}\left[\mathbf{X}\left(\mathbf{X}^{\mathrm{T}} \mathbf{X}\right)^{-1} \mathbf{X}^{\mathrm{T}}-\mathbf{X} \mathbf{C}\left(\mathbf{C}^{\mathrm{T}} \mathbf{X}^{\mathrm{T}} \mathbf{X}\right)^{-1} \mathbf{C}^{\mathrm{T}} \mathbf{X}^{\mathrm{T}}\right] \mathbf{Y} \cdot(2.24)
$$

Como a matriz (2.24) também é idempotente de posto q-d, temos ainda que

$$
\frac{\mathrm{Q}}{\sigma^{2}} \sim \chi_{\mathrm{q}-\mathrm{d}}^{2}
$$

Como as matrizes das formas quadráticas de $Q$ e $\mathrm{SQR}_{1}$ são mutuamente ortogonais, ou seja, $\left[\mathbf{I}-\mathbf{X}\left(\mathbf{X}^{\mathrm{T}} \mathbf{X}\right)^{-1} \mathbf{X}^{\mathrm{T}}\right]\left[\mathbf{X}\left(\mathbf{X}^{\mathrm{T}} \mathbf{X}\right)^{-1} \mathbf{X}^{\mathrm{T}}-\mathbf{X} \mathbf{C}\left(\mathbf{C}^{\mathrm{T}} \mathbf{X}^{\mathrm{T}} \mathbf{X} \mathbf{C}\right)^{-1} \mathbf{C}^{\mathrm{T}} \mathbf{X}^{\mathrm{T}}\right]=0$, então Q é independente de $\mathrm{SQR}_{1}$. Portanto, 


$$
\mathrm{F}=\frac{\mathrm{Q}}{\mathrm{SQR}_{1}} \frac{\mathrm{n}-2 \mathrm{q}-2}{\mathrm{q}-\mathrm{d}}
$$

segue uma distribuição F-Snedecor com (q-d) e (n-2q-2) graus de liberdade.

A estatística (2.26) é adequada para avaliar a magnitude do aumento que ocorre na soma de quadrados residual quando o modelo restrito, que possui um número menor de parâmetros, é adotado no lugar de um modelo sem restrições. Assim, a hipótese nula será rejeitada se o valor desta estatística superar o valor crítico, ou seja, se

$$
\mathrm{F}>\mathrm{F}_{\alpha} \text {, }
$$

onde $\mathrm{F}_{\alpha}$ é o quantil de ordem 1- $\alpha$ da distribuição F-Snedecor com (q-d) e (n-2q-2) graus de liberdade. Assim, o modelo com restrição é rejeitado em favor de um modelo irrestrito se a diminuição no número de parâmetros ocasionada pelas restrições, implicar em um aumento significativo na soma de quadrados residual.

\subsection{Modelos aditivos generalizados com defasagens distribuídas}

Os modelos aditivos generalizados (MAG) assumem que a média de uma variável resposta $\mathrm{Y}$ pode ser escrita como a soma de funções não especificadas das variáveis preditoras $X_{r}, r=1, \ldots, p$ (Hastie e Tibishirani, 1990). Para uma variável resposta com distribuição normal, o modelo é da forma

$$
\mu=E(Y)=\alpha+\sum_{r=1}^{p} f_{r}\left(X_{r}\right)=\eta,
$$


onde $\alpha$ é um parâmetro desconhecido e $\mathrm{f}_{\mathrm{r}}($.$) são funções suaves não especificadas. De$ uma forma mais geral, a variável resposta segue uma distribuição que pertence à família exponencial, não necessariamente normal, isto é, a sua função densidade de probabilidade ou função de probabilidade é da forma

$$
\mathrm{f}(\mathrm{y}, \theta, \phi)=\exp (\phi(\mathrm{y} \theta-\mathrm{b}(\theta))+\mathrm{c}(\mathrm{y}, \phi))
$$

onde $b($.$) e c(.) são funções conhecidas, b(.) é duas vezes diferenciável, e \phi^{-1}>0$ é o parâmetro de dispersão que neste trabalho suporemos ser conhecido. Tem-se que $\mathrm{E}(\mathrm{Y})$ $=\mu=b^{\prime}(\theta)$ e $\operatorname{Var}(Y)=\phi^{-1} b^{\prime \prime}(\theta)$.

Assim como nos modelos lineares generalizados (McCullagh e Nelder, 1989), a média de $Y, \mu$, está relacionada com um conjunto de variáveis preditoras $X_{1}, \ldots, X_{n}$ por meio de uma função de ligação g(.) (monótona, duas vezes diferenciável) isto é,

$$
g(\mu)=\eta
$$

sendo, porém, $\eta$ o preditor aditivo

$$
\eta=\alpha+\sum_{r=1}^{p} f_{r}\left(X_{r}\right)
$$

Este preditor corresponde a um modelo totalmente não paramétrico. O termo "aditivo" deve-se ao fato do modelo (2.32) ser constituído pela soma de funções das variáveis preditoras, o que permite avaliar o efeito de cada uma delas na variável resposta, condicionalmente à presença das outras variáveis no modelo.

O procedimento mais utilizado para ajustar as funções suaves em um MAG é o retroajuste, que é um procedimento que se assemelha ao algoritmo de Gauss-Seidel 
(Golub e Van Loan, 1983) para a solução, de forma iterativa, de sistemas de equações lineares. Para dar uma pequena noção do que seja o retroajuste, falaremos de um modelo simples contendo apenas duas variáveis preditoras e variável resposta com distribuição normal. Suponha que dispomos de valores iniciais $\hat{\alpha}$ para $\alpha$ e $\hat{f}_{2}($.) para $f_{2}($.$) . Uma estimativa \hat{f}_{1}($.$) da função suave f_{1}($.$) pode ser encontrada suavizando o$ resíduo parcial y - $\hat{\alpha}-\hat{f}_{2}\left(X_{2}\right)$ por meio de algum método de suavização como, por exemplo, o loess ou o cubic spline (ver Hastie e Tibshirani, 1990, por exemplo). Como temos apenas uma aproximação para $\mathrm{f}_{2}($.$) , então a estimativa dessa função pode$ ser melhorada suavizando y $-\hat{\alpha}-\hat{f}_{1}\left(X_{1}\right)$. Este processo é repetido até que um ajuste suave se estabeleça. Geralmente a convergência é obtida, mesmo com estimativas iniciais dadas por $\hat{\mathrm{f}}_{1}()=.\hat{\mathrm{f}}_{2}()=$.0 . O algoritmo de retroajuste é facilmente generalizado para mais de duas variáveis preditoras: melhorar $\hat{f}_{k}($.$) suavizando y-\hat{\alpha}-\sum_{r \neq k} \hat{f}_{r}\left(X_{r}\right)$, repetindo o processo para todo $r$ até ocorrer convergência.

Para dados não-normais, suavizadores ponderados são adotados (ver, por exemplo, Lima et al., 2001). Sejam $Y_{1}, \ldots, Y_{n} n$ variáveis aleatórias independentes cada uma com função densidade probabilidade ou função de probabilidade na forma (2.31) $\operatorname{com} \theta=\theta_{\mathrm{i}}, \mathrm{i}=1, \ldots, \mathrm{n}$. Suponha que a média de $\mathrm{Y}_{\mathrm{i}}, \mu_{\mathrm{i}}$, está relacionada com um conjunto de variáveis preditoras (não aleatórias) $X_{1}, \ldots, X_{p}$ através de uma função monótona e diferenciável

$$
\mathrm{g}\left(\mu_{\mathrm{i}}\right)=\eta_{\mathrm{i}}
$$

denominada função de ligação, onde 


$$
\eta_{\mathrm{i}}=\alpha+\mathrm{f}_{1}\left(\mathrm{x}_{\mathrm{il}}\right)+\ldots+\mathrm{f}_{\mathrm{p}}\left(\mathrm{x}_{\mathrm{ip}}\right)
$$

com $x_{i 1}, \ldots, x_{i p}$ sendo os valores observados de $X_{1}, \ldots, X_{p}$ no i-ésimo elemento da amostra. O ajuste do modelo é feito combinando-se dois procedimentos iterativos: o de ponderação local, bastante similar ao de ponderação de Fisher adotado nos MLG, e o retroajuste. Em cada iteração do algoritmo de ponderação local ocorre um procedimento de retroajuste da forma esquematizada no algoritmo apresentado a seguir. O processo é iniciado fazendo $\alpha^{(0)}=\mathrm{g}(\overline{\mathrm{y}})$ e $\mathbf{f}_{1}^{(0)}=\ldots=\mathbf{f}_{\mathrm{p}}^{(0)}=\mathbf{0}$. O algoritmo consiste em iterar os seguintes passos para $\mathrm{m}=1,2, \ldots$ :

Passo 1: para $\mathrm{i}=1, \ldots, \mathrm{n}$, calcular

$$
z_{i}^{(m)}=\eta_{i}^{(m)}+\left(y_{i}-\mu_{i}^{(m)}\right)\left(\frac{\partial \eta_{i}}{\partial \mu_{i}}\right)_{(m)},
$$

onde $\eta_{\mathrm{i}}^{(\mathrm{m})}=\alpha^{(\mathrm{m}-1)}+\sum_{\mathrm{r}=1}^{\mathrm{p}} \mathbf{f}_{\mathrm{r}}^{(\mathrm{m}-1)}\left(\mathrm{x}_{\mathrm{r}}\right)$ e $\mu_{\mathrm{i}}^{(\mathrm{m})}=\mathrm{g}^{-1}\left(\eta_{\mathrm{i}}^{(\mathrm{m})}\right)$,

$$
\mathrm{w}_{\mathrm{i}}^{(\mathrm{m})}=\left[\left(\frac{\partial \mu_{i}}{\partial \eta_{i}}\right)^{(\mathrm{m})}\right]^{2}\left(\mathrm{~V}_{\mathrm{i}}^{(\mathrm{m})}\right)^{-1}, \text { onde } \mathrm{V}_{\mathrm{i}}^{(\mathrm{m})}=\left(\frac{\partial \mu_{\mathrm{i}}}{\partial \theta_{i}}\right)^{(\mathrm{m})}
$$

e a matriz

$S_{\mathrm{r}}^{(\mathrm{m})}$, denominada matriz suavizadora ponderada de dimensão (nxn) relativa à r-ésima covariável, com matriz de pesos $\quad W^{(m)}=\operatorname{diagonal}\left(w_{1}^{(m)}, \ldots, w_{n}^{(m)}\right), r=$ $1, \ldots$, p. Esta matriz depende do método de suavização adotado. 
Passo 2: (Retroajuste)

Fazer $\alpha^{(\mathrm{m})}=\overline{\mathrm{z}}^{(\mathrm{m})}=\sum_{\mathrm{i}=1}^{\mathrm{n}} \frac{\mathrm{z}_{\mathrm{i}}^{(\mathrm{m})}}{\mathrm{n}}, \mathbf{f}_{\mathrm{r}(\mathrm{m})}^{(\mathrm{m})}=\mathbf{f}_{\mathrm{r}}^{(\mathrm{m}-1)}, \mathrm{r}=1, \ldots, \mathrm{p}$ e calcular

$$
\mathbf{f}_{\mathbf{r}(v)}^{(\mathrm{m})}=\mathbf{S}_{\mathrm{r}}^{(\mathrm{m})} \mathbf{r}_{\mathrm{r}(\mathrm{r})}^{(\mathrm{m})}, \mathrm{r}=1, \ldots, \mathrm{p}
$$

onde $\mathbf{r}_{\mathrm{r}(\mathrm{v})}^{(\mathrm{m})}=\left(\mathrm{r}_{\mathrm{lr}(\mathrm{v})}^{(\mathrm{m})}, \ldots, \mathrm{r}_{\mathrm{nr}(\mathrm{r})}^{(\mathrm{m})}\right)^{\mathrm{T}}$ é o vetor de resíduos parciais com elementos dados por

$$
\mathrm{r}_{\mathrm{ir}(\mathrm{v})}^{(\mathrm{m})}=\mathrm{z}_{\mathrm{i}}^{(\mathrm{m})}-\overline{\mathrm{z}}^{(\mathrm{m})}-\sum_{\mathrm{k}=1}^{\mathrm{r}-1} \mathrm{f}_{\mathrm{k}(\mathrm{v})}^{(\mathrm{m})}\left(\mathrm{x}_{\mathrm{ik}}\right)-\sum_{\mathrm{k}=\mathrm{r}+\mathrm{k}}^{\mathrm{p}} \mathrm{f}_{\mathrm{k}(\mathrm{v}-1)}^{(\mathrm{m})}\left(\mathrm{x}_{\mathrm{ik}}\right),
$$

para $v=1,2, \ldots$, até que

$$
\left\|\mathbf{f}_{\mathrm{r}(\mathrm{v})}^{(\mathrm{m})}-\mathbf{f}_{\mathrm{r}(\mathrm{v}-1)}^{(\mathrm{m})}\right\| \leq \varepsilon, \mathrm{r}=1, \ldots, \mathrm{p},
$$

para um valor de $\varepsilon>0$ fixado. Nesse ponto,

$$
\mathbf{f}_{\mathrm{r}}^{(\mathrm{m})}=\mathbf{f}_{\mathrm{r}(\mathrm{v})}^{(\mathrm{m})} \text {. }
$$

Passo 3: Repetir os passos 1 e 2 até que

$$
\frac{\sum_{\mathrm{r}=1}^{\mathrm{p}}\left\|\mathbf{f}_{\mathrm{r}}^{(\mathrm{m})}-\mathbf{f}_{\mathrm{r}}^{(\mathrm{m}-1)}\right\|}{\sum_{\mathrm{r}=1}^{\mathrm{p}}\left\|\mathbf{f}_{\mathrm{r}}^{(\mathrm{m}-1)}\right\|} \leq \xi
$$

para um valor de $\xi>0$ fixado.

A motivação para utilização desse algoritmo na estimação de um modelo aditivo generalizado pode ser encontrada em Hastie e Tibshirani (1990) e Marx e Eilers (1998). 
Dependendo do método de suavização adotado, as soluções obtidas correspondem a estimativas que maximizam uma versão penalizada da função de verossimilhança de Y. Denotando por $l\left(y_{i} ; \eta_{i}\right)$ o logaritmo da função de verossimilhança escrita como uma função de $\eta_{i}=\alpha+\sum_{r=1}^{p} f_{r}\left(X_{i r}\right), i=1, \ldots, n$, uma possível versão penalizada da função de verossimilhança é

$$
\sum_{\mathrm{i}=1}^{\mathrm{n}} l\left(\mathrm{y}_{\mathrm{i}}, \eta_{\mathrm{i}}\right)-\frac{1}{2} \sum_{\mathrm{r}=1}^{\mathrm{p}} \lambda_{\mathrm{r}} \int\left(\mathrm{f}_{\mathrm{r}}^{\prime \prime}(\mathrm{u})\right)^{2} \mathrm{du}
$$

onde $\lambda_{\mathrm{r}}$ é o parâmetro de suavização fixado para estimar $\mathrm{f}_{\mathrm{r}}, \mathrm{r}=1, \ldots$, p. O termo de penalidade $\int\left(f_{r}^{\prime \prime}(u)\right)^{2} d u$ mede a ondulação da função $f_{r}$. No caso de $f_{r}$ ser uma reta, $\int\left(f_{r}^{\prime \prime}(u)\right)^{2} d u=0$. Os parâmetros $\lambda_{r}$ regulam a relação de ganho e perda entre o viés e a ondulação das curvas estimadas. Valores grandes de $\lambda_{\mathrm{r}}$ produzem curvas mais suaves, isto é, menos onduladas. Pode-se mostrar que as soluções $\hat{f}_{1}, \ldots, \hat{f}_{p}$ que maximizam (2.34) correspondem às funções suavizadas obtidas pelo método cubic spline.

Também fazem parte dos MAG modelos cujo preditor aditivo combina formas paramétricas de algumas variáveis preditoras com não paramétricas de outras, constituindo os MAG semi-paramétricos. Variáveis defasadas no tempo podem também ser adicionadas ao modelo (2.32) obtendo-se um modelo aditivo generalizado com defasagens distribuídas. Uma forma bastante geral para este modelo, dada em Zanobetti et al.(2000) é:

$$
g\left[E\left(y_{1}\right)\right]=\alpha+\psi^{T} \bar{b}_{t}+\sum_{r=1}^{p} f_{r}\left(s_{r t}\right)+\sum_{j=0}^{q} \beta_{j} x_{t-j}, \quad t=q+1, \ldots, n,
$$


onde

g(.) é uma função de ligação;

yt é o valor da variável resposta no instante de tempo t;

$\alpha$ é o parâmetro correspondente ao intercepto;

$\psi^{\mathrm{T}}=\left(\psi_{1} \cdots \psi_{\mathrm{h}}\right)$ é o vetor de coeficientes (desconhecidos) das h variáveis preditoras modeladas de forma linear;

$\mathbf{b}_{\mathrm{t}}=\left(\mathrm{b}_{\mathrm{t} 1} \cdots \mathrm{b}_{\mathrm{th}}\right)^{\mathrm{T}}$ é o vetor que contém os valores das $\mathrm{h}$ variáveis preditoras modeladas linearmente, observados no tempo t;

$\mathrm{f}_{1}, \ldots, \mathrm{f}_{\mathrm{r}}$ são funções não especificadas;

$\mathrm{S}_{\mathrm{rt}}$ é o valor da r-ésima variável preditora, modelada de forma não paramétrica, observado no instante t;

$\beta_{\mathrm{j}}$ é o coeficiente desconhecido associado ao valor da variável preditora $\mathrm{X}$ defasada de j unidades de tempo em relação ao instante $t, j=0, \ldots, q, t=q+1, \ldots, n$;

$\mathrm{x}_{\mathrm{t}-\mathrm{j}}$ é o valor observado de uma variável preditora $\mathrm{X}$ defasada de $\mathrm{j}$ unidades de tempo em relação ao instante $\mathrm{t}$.

O ajuste desses modelos é feito de forma similar aos totalmente nãoparamétricos e também é descrito em Lima et al. (2001).

Testes de hipotése sobre os parâmetros dos MAG são feitos de forma similar aos dos MLG.

Suponha que $\eta_{1}$ e $\eta_{2}$ sejam preditores lineares de dois MAG, por exemplo, dados por

$$
\eta_{1}=\alpha+\psi_{1} b_{t 1}+f_{1}\left(s_{1 t}\right)+\ldots+f_{p}\left(s_{p t}\right)+\beta_{0} X_{t} e
$$




$$
\eta_{2}=\alpha+\psi_{1} b_{t 1}+f_{1}\left(s_{1 t}\right)+\ldots+f_{p}\left(s_{p t}\right)+\beta_{0} X_{t}+\ldots+\beta_{q} X_{t-q}
$$

Para testar

$$
\mathrm{H}_{0}: \beta_{1}=\ldots=\beta_{\mathrm{q}}=0 \text {, }
$$

a partir do ajuste dos modelos com preditores aditivos dados em (2.36) e (2.37), obtemos primeiramente os desvios, definidos da mesma forma que para os modelos lineares generalizados, ou seja,

$$
\begin{aligned}
& \mathrm{D}\left(\mathbf{y}, \widehat{\boldsymbol{\eta}}_{1}\right)=2\left\{\log \left(\boldsymbol{\eta}_{\text {max }} ; \mathbf{y}\right)-\log \left(\hat{\boldsymbol{\eta}}_{\mathbf{1}} ; \mathbf{y}\right)\right\} \mathrm{e} \\
& \mathrm{D}\left(\mathbf{y}, \widehat{\boldsymbol{\eta}}_{\mathbf{2}}\right)=2\left\{\log \left(\boldsymbol{\eta}_{\text {max }} ; \mathbf{y}\right)-\log \left(\hat{\boldsymbol{\eta}}_{2} ; \mathbf{y}\right)\right\},
\end{aligned}
$$

onde $\log \left(\eta_{\max } ; \mathbf{y}\right)$ é o valor do logaritmo da função verossimilhança calculada no modelo saturado, isto é, com n-q parâmetros, e $\log \left(\hat{\boldsymbol{\eta}}_{1} ; \mathbf{y}\right)$ e $\log \left(\hat{\boldsymbol{\eta}}_{2} ; \mathbf{y}\right)$ são os logaritmos das funções de verossimilhança calculadas sob os modelos (2.36) e (2.37) ajustados, respectivamente.

A estatística

$$
\phi D\left(\widehat{\boldsymbol{\eta}}_{2}, \widehat{\boldsymbol{\eta}}_{1}\right)=\mathrm{D}\left(\mathbf{y}, \widehat{\boldsymbol{\eta}}_{1}\right)-\mathrm{D}\left(\mathbf{y}, \widehat{\boldsymbol{\eta}}_{2}\right)
$$

é utilizada para o teste de $\mathrm{H}_{0}$, adotando-se como referência aproximada uma distribuição $\chi^{2}$ com número de graus de liberdade dado por

$$
\operatorname{gl}\left(\hat{\boldsymbol{\eta}}_{1}\right)-\operatorname{gl}\left(\hat{\boldsymbol{\eta}}_{2}\right)=\operatorname{traço}\left(2 \mathbf{R}_{1}-\mathbf{R}_{1}^{\mathrm{T}} \hat{\mathbf{W}}_{1} \mathbf{R}_{1} \hat{\mathbf{W}}_{1}^{-1}\right)-\operatorname{traço}\left(2 \mathbf{R}_{2}-\mathbf{R}_{2}^{\mathrm{T}} \hat{\mathbf{W}}_{2} \mathbf{R}_{2} \hat{\mathbf{W}}_{2}^{-1}\right)
$$

onde $\mathbf{R}_{1}$ e $\mathbf{R}_{2}$ são os operadores de ajuste aditivo ponderado, obtidos no último passo do algoritmo de ponderação local, e são tais que $\hat{\boldsymbol{\eta}}_{1}=\mathbf{R}_{1} \mathbf{z}$ e $\hat{\boldsymbol{\eta}}_{2}=\mathbf{R}_{2} \mathbf{z}$, respectivamente. Já $\hat{W}_{1}$ e $\hat{W}_{2}$ são matrizes diagonais cujos elementos são os pesos $W_{i}$ calculados na convergência do algoritmo de ponderação local no ajuste dos modelos 
com preditores lineares dados em (2.36) e (2.37), respectivamente. Valores grandes de $\phi D\left(\hat{\boldsymbol{\eta}}_{2}, \hat{\boldsymbol{\eta}}_{1}\right)$ sugerem que a contribuição dos termos presentes apenas em $\eta_{2}$ é significativa.

Um critério de ajuste adotado nos MAG é a estatística AIC, inspirada no critério de informação de Akaike; quanto menor o valor do AIC, melhor será considerado o ajuste. A expressão dessa estatística é

$$
\mathrm{AIC}=\frac{1}{\mathrm{n}-\mathrm{q}} \mathrm{D}(\mathbf{y} ; \hat{\boldsymbol{\eta}})+\frac{2}{\mathrm{n}-\mathrm{q}} \operatorname{traço}(\mathbf{R}) \phi,
$$

onde $\mathrm{D}(\mathbf{y} ; \mathfrak{\eta})$ é o desvio do modelo sob investigação e $\mathbf{R}$ é uma matriz que é construída durante o processo de estimação do modelo, e é tal que $\hat{\boldsymbol{\eta}}=\mathbf{R z}$, onde $\mathbf{z}$ é a variável dependente modificada construída no último passo do algoritmo de ponderação local.

A estatística AIC é também utilizada como critério na seleção dos parâmetros de suavização, sendo escolhida a combinação dos parâmetros que minimiza o valor dessa estatística. Outro critério adotado com a mesma finalidade é o da validação cruzada, que tem expressão aproximada por

$$
\mathrm{VC}=\frac{\mathrm{D}(\mathbf{y}, \hat{\boldsymbol{\eta}}) /(\mathrm{n}-\mathrm{q})}{[1-\operatorname{traço}(\mathbf{R}) /(\mathrm{n}-\mathrm{q})]^{2}},
$$

onde $\mathrm{D}(\mathbf{y}, \hat{\boldsymbol{\eta}})$ e $\mathbf{R}$ são definidos da mesma forma que para o AIC (Hastie e Tibshirani, 1990). São selecionados os parâmetros de suavização que minimizam essa expressão.

No próximo capítulo será ilustrada a estimação de um MAG da forma (2.35), considerando o suavizador P-spline (Eilers e Marx, 1996 ou Marx e Eilers, 1998) na estimação das funções não especificadas. 
Capítulo 3

\section{Modelos com defasagens distribuídas com restrição não paramétrica}

\subsection{Introdução}

As restrições impostas aos coeficientes do modelo linear com defasagens distribuídas dadas na equação (2.3) estabelecem de forma rígida que a relação entre os parâmetros $\beta_{\mathrm{j}}$ e as defasagens $\mathrm{j}, \mathrm{j}=0, \ldots, \mathrm{q}$, é descrita por um polinômio. Entretanto, esse tipo de relação pode não ser adequado para modelar estruturas mais locais, quando são considerados longos períodos de defasagem. Isto ocorre, por exemplo, em Epidemiologia Ambiental, no estudo do efeito colheita descrito, entre outros, por Zanobetti et al.(2000). Esses autores propuseram, então, que a relação entre os coeficientes e as defasagens seja descrita de uma forma mais flexível por meio de uma regressão spline (Hastie e Tibshirani, 1990). De uma forma geral, esse método não paramétrico de modelagem, descrito na seção 3.2, assume que a relação entre a média da variável resposta Y e uma variável preditora X é descrita por "pedaços" de polinômios que se unem em uma sequência de nós, $\delta_{1}, \ldots, \delta_{K}$, de valores de $X$ como ilustrado na Figura 3.1.

Uma dificuldade que surge ao se adotar esse método de modelagem é a escolha adequada do número e posição dos nós. Um número muito grande de nós implica em uma curva ajustada pouco suave, enquanto que a utilização de um número pequeno de nós pode esconder comportamentos locais. Uma forma de contornar esse problema é a 
utilização do suavizador P-spline, proposto por Eilers e Marx (1996), no qual um termo de penalidade é adicionado ao critério de ajuste de forma a poder controlar a suavidade da curva estimada, independentemente do número e posição dos nós. Esse suavizador também é apresentado na seção 3.2. A aplicação desse método de suavização na estimação de modelos lineares e modelos aditivos generalizados com defasagens distribuídas está ilustrada nas seções 3.3 e 3.4, respectivamente.

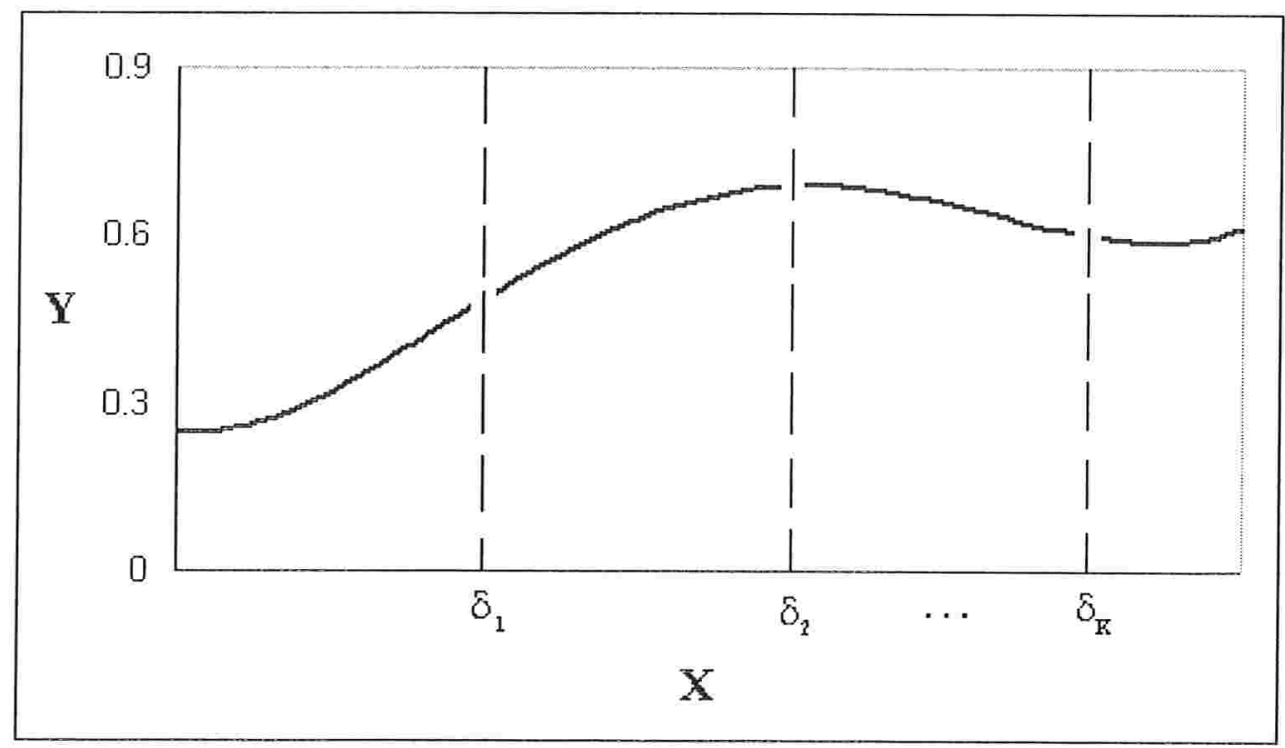

Figura 3.1: Ilustração de uma regressão spline

\subsection{Regressão spline e o suavizador $P$-spline}

Suponhamos que $\left(\mathrm{x}_{\mathrm{i}}, \mathrm{y}_{\mathrm{i}}\right), \mathrm{i}=1, \ldots, \mathrm{n}$, constituem o conjunto de valores observados de uma variável preditora $X$ e da variável resposta $Y$, e que

$$
y_{i}=f\left(x_{i}\right)+e_{i},
$$


onde $f\left(x_{i}\right)=E\left(Y_{i} \mid x_{i}\right)$ é uma função suave e e são erros com média 0 , variância constante $\sigma^{2}$ e independentes.

A regressão spline assume que a função $\mathrm{f}(\mathrm{x})$ consiste em uma sequência de polinômios de grau d que se unem em determinados valores de $\mathrm{X}$ denominados nós ou pontos de quebra. Em geral, fixa-se $d=3$ e assume-se que a função $\mathrm{f}$ possui primeira e segunda derivadas contínuas nos nós.

Formalmente, dados os $\mathrm{K}$ nós $\delta_{1}, \ldots, \delta_{\mathrm{K}}$, assume-se que $\mathrm{f}(\mathrm{x})$ pertence ao espaço gerado por um conjunto de funções de base $B_{j}(x), j=1, \ldots, K+d+1$. Diferentes funções de base podem ser consideradas. Uma possível escolha é a base "série de potência truncada", formada pelas seguintes funções:

$$
\begin{gathered}
\mathrm{B}_{0}(\mathrm{x})=1, \\
\mathrm{~B}_{1}(\mathrm{x})=\mathrm{x}, \\
\vdots \\
\mathrm{B}_{\mathrm{d}}(\mathrm{x})=\mathrm{x}^{\mathrm{d}}, \\
\mathrm{B}_{\mathrm{d}+1}(\mathrm{x})=\left(\mathrm{x}-\delta_{1}\right)_{+}^{\mathrm{d}}, \\
\vdots \\
\mathrm{B}_{\mathrm{K}+\mathrm{d}+1}(\mathrm{x})=\left(\mathrm{x}-\delta_{\mathrm{K}}\right)_{+}^{\mathrm{d}},
\end{gathered}
$$

onde $\left(x-\delta_{i}\right)_{+}^{d}=\left\{\begin{array}{ll}\left(x-\delta_{i}\right)^{d}, & \text { se } x-\delta_{i} \geq 0 \\ 0, & \text { se } x-\delta_{i}<0\end{array}, \quad i=1, \ldots, K\right.$.

Esta base fornece a seguinte expressão paramétrica para $\mathrm{f}(\mathrm{x})$ :

$$
f(x)=\beta_{0}+\beta_{1} x+\ldots+\beta_{d} x^{d}+\sum_{i=1}^{K} v_{i}\left(x-\delta_{i}\right)_{+}^{d},
$$


onde $\beta_{0}, \beta_{1}, \ldots, \beta_{\mathrm{d}}, v_{1}, \ldots, v_{\mathrm{K}}$ são parâmetros a serem estimados.

Calculando a matriz de planejamento X, cuja i-ésima linha é dada por

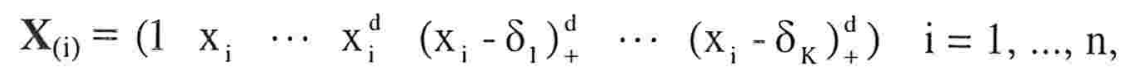

as estimativas dos parâmetros do modelo (3.2) podem ser obtidas pelo método de mínimos quadrados, ou seja, determina-se, dentre todas as possíveis escolhas para o vetor de parâmetros $\beta=\left(\beta_{0}, \ldots, \beta_{\mathrm{d}}, v_{1}, \ldots, v_{\mathrm{K}}\right)^{\mathrm{T}}$, aquela que minimiza

$$
\sum_{i=1}^{n}\left[y_{i}-\left(\beta_{0}+\beta_{1} x+\ldots+\beta_{d} x^{d}+\sum_{i=1}^{K} v_{i}\left(x-\delta_{i}\right)_{+}^{d}\right)\right]^{2} .
$$

Tem-se então que

$$
\hat{\boldsymbol{\beta}}=\left(\mathbf{X}^{\mathrm{T}} \mathbf{X}\right)^{-1} \mathbf{X}^{\mathrm{T}} \mathbf{Y} .
$$

Segundo Hastie e Tibshirani(1990), outras bases podem ser consideradas, como, por exemplo, a base $B$-spline descrita em de Boor (1978) e Marx e Eilers (1998).

Qualquer que seja a base escolhida, o grande atrativo da regressão spline é a sua simplicidade computacional. Métodos padrão de ajuste de modelos de regressão, como o método de mínimos quadrados adotado nesta seção, podem ser utilizados. A utilização da regressão spline no ajuste de um modelo aditivo generalizado implica em uma relevante simplicidade computacional, como será visto na seção 3.3 .

Um problema na utilização da regressão spline está em ter que se escolher o número e posição dos nós de forma adequada: um número muito grande de nós fará com que a curva ajustada apresente variabilidade maior do que a justificada pelos dados, enquanto que um número pequeno implica em um viés da curva estimada, como descrito em Hastie e Tibshirani (1990). Não iremos entrar em detalhes neste 
trabalho sobre o problema da escolha do número de nós. Um levantamento dos trabalhos que abordam esse problema é apresentado em Ruppert (2001). Vamos adotar a recomendação feita por Marx e Eilers (1998): um número grande de nós igualmente espaçados é escolhido e, para evitar que a curva ajustada interpole os dados, um termo de penalidade é adicionado ao critério de ajuste. Esta foi a estratégia seguida por Zanobetti et. al. (2000). A obtenção de uma função ajustada pouco suave foi evitada, restrigindo-se a magnitude dos coeficientes $v_{\mathrm{i}}$ em (3.4). Isto é alcançado adicionandose o termo de penalidade $\lambda \sum_{i=1}^{K} v_{i}^{2}$, onde $\lambda>0$ é o parâmetro de suavização, ao critério dos mínimos quadrados. Assim a estimativa do vetor de parâmetros $\boldsymbol{\beta}$ é obtida minimizando:

$$
\sum_{i=1}^{n}\left[y_{i}-\left(\beta_{0}+\beta_{1} x+\ldots+\beta_{d} x^{d}+\sum_{i=1}^{K} v_{i}\left(x-\delta_{i}\right)_{+}^{d}\right)\right]^{2}+\lambda^{2} \sum_{i=1}^{K} v_{i}^{2} .
$$

Esta estratégia de estimação é conhecida como método spline penalizado ou $P$-spline. O parâmetro de suavização é responsável por regular a suavização da curva: quanto maior o valor de $\lambda$, menores os valores de $v_{i}, i=1, \ldots, \mathrm{K}$, e mais a curva ajustada se aproxima de um polinômio de grau d. Por outro lado, quando $\lambda \rightarrow 0$, o termo de penalidade desaparece de (3.5) e tem-se novamente o problema da escolha do número e posição dos nós. Critérios para a seleção de um valor adequado para $\lambda$ serão apresentados na seção 3.4 . 


\subsection{Utilização do $P^{3}$ spline no ajuste de modelos com defasagens distribuídas}

No modelo com defasagens distribuídas dado na equação (2.1) foram impostas aos coeficientes as restrições polinomiais

$$
\beta_{\mathrm{j}}=\sum_{\mathrm{k}=0}^{\mathrm{d}} \tau_{\mathrm{k}} \mathrm{j}^{\mathrm{k}}, \quad \mathrm{j}=0, \ldots, \mathrm{q}
$$

Zanobetti et. al. (2000) consideraram que modelos polinomiais podem não ser adequados para modelar estruturas locais e propuseram que a relação entre os parâmetros do modelo e a defasagem seja descrita por uma regressão spline, ou seja, propuseram que (3.6) seja substituída por

$$
\beta_{j}=\sum_{k=0}^{d} \tau_{k} j^{k}+\sum_{i=1}^{K} v_{i}\left(j-\delta_{i}\right)_{+}^{d} j=0, \ldots, q,
$$

onde $\delta_{\mathrm{i}}$ são valores das defasagens correspondentes aos nós.

Para evitar que a estimação dos coeficientes pelo método de mínimos quadrados produza funções muito onduladas, o método de suavização P-spline foi adotado. Assim, para estimar os parâmetros do modelo (2.1) foi adotado o seguinte critério:

$$
\operatorname{minimizar} \sum_{\mathrm{t}=\mathrm{q}+1}^{\mathrm{n}}\left(\mathrm{y}_{\mathrm{t}}-\alpha-\beta_{0} \mathrm{x}_{\mathrm{t}}-\ldots-\beta_{\mathrm{q}} \mathrm{x}_{\mathrm{t}-\mathrm{q}}\right)^{2}+\lambda^{2} \sum_{\mathrm{i}=1}^{\mathrm{K}} \mathrm{v}_{\mathrm{i}}^{2} \text {, }
$$

onde $\beta_{\mathrm{j}}, \mathrm{j}=0, \ldots, \mathrm{q}$, é dado em (3.7), ou seja,

$$
\begin{aligned}
& \beta_{0}=\tau_{0}+v_{1}\left(0-\delta_{1}\right)_{+}^{\mathrm{d}}+v_{2}\left(0-\delta_{2}\right)_{+}^{\mathrm{d}}+\ldots+v_{\mathrm{K}}\left(0-\delta_{\mathrm{K}}\right)_{+}^{\mathrm{d}} \\
& \beta_{1}=\tau_{0}+\tau_{1}+\tau_{2} \ldots+\tau_{\mathrm{d}}+v_{1}\left(1-\delta_{1}\right)_{+}^{\mathrm{d}}+v_{2}\left(1-\delta_{2}\right)_{+}^{\mathrm{d}}+\ldots+v_{\mathrm{K}}\left(1-\delta_{\mathrm{K}}\right)_{+}^{\mathrm{d}} \\
& \beta_{2}=\tau_{0}+2 \tau_{1}+2^{2} \tau_{2}+\ldots+2^{\mathrm{d}} \tau_{\mathrm{d}}+v_{1}\left(2-\delta_{1}\right)_{+}^{\mathrm{d}}+v_{2}\left(2-\delta_{2}\right)_{+}^{\mathrm{d}}+\ldots+v_{\mathrm{K}}\left(2-\delta_{\mathrm{K}}\right)_{+}^{\mathrm{d}}
\end{aligned}
$$




$$
\beta_{\mathrm{q}}=\tau_{0}+\mathrm{q} \tau_{1}+\mathrm{q}^{2} \tau_{2}+\ldots+\mathrm{q}^{\mathrm{d}} \tau_{\mathrm{d}}+v_{1}\left(\mathrm{q}-\delta_{1}\right)_{+}^{\mathrm{d}}+v_{2}\left(\mathrm{q}-\delta_{2}\right)_{+}^{\mathrm{d}}+\ldots+v_{\mathrm{K}}\left(\mathrm{q}-\delta_{\mathrm{K}}\right)_{+}^{\mathrm{d}},
$$

$v_{1}, \ldots, v_{K}$ são parâmetros desconhecidos e $\lambda$ é o parâmetro de suavização a ser fixado.

O vetor de parâmetros $\beta=\left(\alpha \beta_{0} \cdots \beta_{\mathrm{q}}\right)^{\mathrm{T}}$ pode então ser expresso como

$$
\beta=S \gamma,
$$

onde $S_{(\mathrm{q}+2) \times(\mathrm{K}+\mathrm{d}+2)}=\left[\begin{array}{cccccccc}1 & 0 & 0 & \cdots & 0 & 0 & \cdots & 0 \\ 0 & 1 & 0 & \cdots & 0^{\mathrm{d}} & \left(0-\delta_{1}\right)_{+}^{\mathrm{d}} & \cdots & \left(0-\delta_{\mathrm{K}}\right)_{+}^{\mathrm{d}} \\ 0 & 1 & 1 & \cdots & 1^{\mathrm{d}} & \left(1-\delta_{1}\right)_{+}^{\mathrm{d}} & \cdots & \left(1-\delta_{\mathrm{K}}\right)_{+}^{\mathrm{d}} \\ \vdots & \vdots & \vdots & \ddots & \vdots & \vdots & \ddots & \vdots \\ 0 & 1 & \mathrm{q} & \cdots & \mathrm{q}^{\mathrm{d}} & \left(\mathrm{q}-\delta_{1}\right)_{+}^{\mathrm{d}} & \cdots & \left(\mathrm{q}-\delta_{\mathrm{K}}\right)_{+}^{\mathrm{d}}\end{array}\right]$

e $\gamma=\left[\begin{array}{lllllllll}\alpha & \tau_{0} & \tau_{1} & \cdots & \tau_{\mathrm{d}} & v_{1} & v_{2} & \cdots & v_{\mathrm{K}}\end{array}\right]^{\mathrm{T}}$.

Utilizando notação matricial, podemos escrever o critério (3.8) como

$$
\operatorname{minimizar}(\mathbf{Y}-\mathbf{X S} \gamma)^{\mathrm{T}}(\mathbf{Y}-\mathbf{X S} \gamma)+\lambda^{2} \gamma^{\mathrm{T}} \mathbf{D} \gamma
$$

onde $\mathbf{Y}$ e $\mathbf{X}$ são como em (2.2), S e $\boldsymbol{\gamma}$ como em (3.9) e $\mathbf{D}=\left[\begin{array}{ll}\mathbf{0}_{(\mathrm{d}+2) \times(\mathrm{d}+2)} & \mathbf{0}_{(\mathrm{d}+2) \times \mathrm{K}} \\ \mathbf{0}_{\mathrm{K \times}(\mathrm{d}+2)} & \mathbf{I}_{\mathrm{K \times K}}\end{array}\right]$.

O estimador de mínimos quadrados penalizados de $\gamma$ é

$$
\hat{\gamma}=\left(\mathbf{S}^{\mathrm{T}} \mathbf{X}^{\mathrm{T}} \mathbf{X} \mathbf{S}+\lambda^{2} \mathbf{D}\right)^{-1} \mathbf{S}^{\mathrm{T}} \mathbf{X}^{\mathrm{T}} \mathbf{Y} \text {. }
$$

Um estimador para $\beta$ é então dado por

$$
\hat{\boldsymbol{\beta}}=\mathbf{S} \hat{\boldsymbol{\gamma}}=\mathbf{S}\left(\mathbf{S}^{\mathrm{T}} \mathbf{X}^{\mathrm{T}} \mathbf{X} \mathbf{S}+\lambda^{2} \mathbf{D}\right)^{-1} \mathbf{S}^{\mathrm{T}} \mathbf{X}^{\mathrm{T}} \mathbf{Y},
$$

e é denominado estimador de defasagens distribuídas spline penalizado de $\beta$. Note que $\hat{\beta}$ depende do parâmetro de suavização $\lambda$. 


\subsection{Utilização do P-spline na estimação de modelos aditivos generalizados com defasagens distribuídas}

Nesta seção vamos ilustrar a utilização do suavizador $P$-spline no ajuste do modelo aditivo generalizado

$$
g\left[E\left(y_{t}\right)\right]=g\left(\mu_{t}\right)=\alpha+\psi^{T} b_{t}+\sum_{r=1}^{p} f_{r}\left(s_{r t}\right)+\sum_{j=0}^{q} \beta_{j} x_{t-j}, t=q+1, \ldots, n,
$$

apresentado na seção 2.4 .

Vamos assumir para isso que as funções não especificadas $\mathrm{f}_{\mathrm{r}}, \mathrm{r}=1, \ldots, \mathrm{p}$, são da forma

$$
\mathrm{f}_{\mathrm{r}}\left(\mathrm{s}_{\mathrm{rt}}\right)=\sum_{\mathrm{k}=1}^{\mathrm{d}_{\mathrm{r}}} \pi_{\mathrm{rk}} \mathrm{s}_{\mathrm{rt}}^{\mathrm{k}}+\sum_{\mathrm{i}=1}^{\mathrm{K}_{\mathrm{r}}} \omega_{\mathrm{ri}}\left(\mathrm{s}_{\mathrm{rt}}-\delta_{\mathrm{r}}\right)_{+}^{\mathrm{d}_{\mathrm{r}}} \quad \mathrm{t}=\mathrm{q}+1, \ldots, \mathrm{n},
$$

onde $\pi_{\mathrm{rk}}, \mathrm{k}=1, \ldots, \mathrm{d}_{\mathrm{r}}, \mathrm{e} \omega_{\mathrm{ri}}, \mathrm{i}=1, \ldots, \mathrm{K}_{\mathrm{r}}$, são coeficientes desconhecidos associados à r-ésima variável modelada de forma não especificada, e $\delta_{\mathrm{ri}}, \mathrm{i}=1, \ldots, \mathrm{K}_{\mathrm{r}}$, são os nós fixados para essa variável. Vamos impor aos parâmetros $\beta_{\mathrm{j}}, \mathrm{j}=0, \ldots, \mathrm{q}$, as restrições (3.7). O vetor de parâmetros a ser estimado é, então, dado por

$$
\begin{aligned}
\gamma^{*}= & {\left[\begin{array}{lllllll}
\alpha & \psi_{1} \cdots \psi_{\mathrm{h}} & \pi_{11} \cdots \pi_{1 \mathrm{~d}_{1}} & \omega_{11} \cdots \omega_{1 \mathrm{~K}_{1}} \cdots \pi_{\mathrm{p} 1} \cdots \pi_{\mathrm{pd}_{\mathrm{p}}} \omega_{\mathrm{p} 1} \cdots \omega_{\mathrm{pK}_{\mathrm{p}}} & \tau_{0} \cdots \tau_{\mathrm{d}} & v_{1} \cdots v_{\mathrm{K}}
\end{array}\right]^{\mathrm{T}} . } \\
& \text { O primeiro passo para a estimação dos parâmetros é construir a matriz de }
\end{aligned}
$$
planejamento $\mathbf{X}$, que para o modelo (3.13) é particionada na forma

$$
\mathbf{X}=\left[1\left|\mathbf{X}_{\mathrm{h}}\right| \mathbf{X}_{\text {suav. }} \mid \mathbf{X}_{\text {def. }}\right]
$$

onde

1 é um vetor cujos (n-q) elementos são iguais a 1; 


$$
\mathbf{X}_{\mathrm{h}}=\left[\begin{array}{c}
\mathbf{b}_{\mathrm{q}+1}^{\mathrm{T}} \\
\vdots \\
\mathbf{b}_{\mathrm{n}}^{\mathrm{T}}
\end{array}\right]
$$

é uma matriz cujas linhas correspondem aos valores das variáveis modeladas linearmente observados em cada instante de tempo;

$$
\begin{aligned}
& \mathbf{X}_{\text {suav. }}=\left[\mathbf{X}_{\text {suav. }, 1}|\ldots| \mathbf{X}_{\text {suav.,p }}\right] \text {, } \\
& \text { onde } \mathbf{X}_{\text {suav }, r}=\left[\begin{array}{cccccc}
\mathrm{s}_{\mathrm{r}, \mathrm{r}+1} & \cdots & \mathrm{s}_{\mathrm{r}, \mathrm{q}+1}^{\mathrm{d}_{r}} & \left(\mathrm{~s}_{\mathrm{r}, q+1}-\delta_{\mathrm{rl}}\right)_{+}^{\mathrm{d}_{r}} & \cdots & \left(\mathrm{s}_{\mathrm{r}, \mathrm{q}+1}-\delta_{\mathrm{rK}}\right)_{r}^{\mathrm{d}_{r}} \\
\vdots & \ddots & \vdots & \vdots & \ddots & \vdots \\
\mathrm{s}_{\mathrm{r}, \mathrm{n}} & \cdots & \mathrm{s}_{\mathrm{r}, \mathrm{n}}^{\mathrm{d}_{r}} & \left(\mathrm{~s}_{\mathrm{r}, \mathrm{n}}-\delta_{\mathrm{rl}}\right)_{+}^{\mathrm{d}_{r}} & \cdots & \left(\mathrm{s}_{\mathrm{r}, \mathrm{n}}-\delta_{\mathrm{rK}}\right)_{+}^{\mathrm{d}_{r}}
\end{array}\right], \quad \mathrm{r}=1, \ldots, \mathrm{p},
\end{aligned}
$$

ou seja, $\mathbf{X}_{\text {suav., r }}$ é a matriz de planejamento associada à r-ésima função não especificada $\mathrm{f}_{\mathrm{r}}, \mathrm{r}=1, \ldots, \mathrm{p}$, cuja t-ésima linha tem a mesma forma da expressão (3.3), a menos do primeiro elemento, e contém os valores das funções de base avaliados em $\mathrm{s}_{\mathrm{rt}}, \mathrm{t}=\mathrm{q}+1$, $\ldots, \mathrm{n}$ e $\mathrm{r}=1, \ldots, \mathrm{p} \mathrm{e}$

$$
\mathbf{X}_{\text {def. }}=\left[\begin{array}{cccc}
\mathrm{x}_{\mathrm{q}+1} & \mathrm{x}_{\mathrm{q}} & \cdots & \mathrm{x}_{1} \\
\vdots & \vdots & \ddots & \vdots \\
\mathrm{x}_{\mathrm{n}} & \mathrm{x}_{\mathrm{n}-1} & \cdots & \mathrm{x}_{\mathrm{n}-\mathrm{q}}
\end{array}\right] \mathbf{U},
$$

onde

$$
\mathbf{U}=\left[\begin{array}{ccccccc}
1 & 0 & \cdots & 0^{\mathrm{d}} & \left(0-\delta_{1}\right)_{+}^{\mathrm{d}} & \cdots & \left(0-\delta_{\mathrm{K}}\right)_{+}^{\mathrm{d}} \\
1 & 1 & \cdots & 1^{\mathrm{d}} & \left(1-\delta_{1}\right)_{+}^{\mathrm{d}} & \cdots & \left(1-\delta_{\mathrm{K}}\right)_{+}^{\mathrm{d}} \\
\vdots & \vdots & \ddots & \vdots & \vdots & \ddots & \vdots \\
1 & \mathrm{q} & \cdots & \mathrm{q}^{\mathrm{d}} & \left(\mathrm{q}-\delta_{1}\right)_{+}^{\mathrm{d}} & \cdots & \left(\mathrm{q}-\delta_{\mathrm{K}}\right)_{+}^{\mathrm{d}}
\end{array}\right],
$$

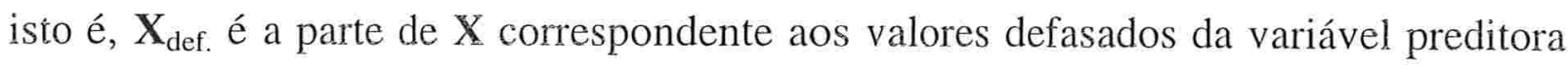
$X$, quando são feitas as restrições (3.7) sobre os coeficientes $\beta_{\mathrm{j}}, \mathrm{j}=\mathrm{q}+1, \ldots, \mathrm{n}$. 
No caso de erros independentes com distribuição normal com média 0 e variância $\sigma^{2}$, o estimador de mínimos quadrados penalizados de $\gamma^{*}$ é dado por

$$
\hat{\gamma}^{*}=\left(\mathbf{X}^{\mathrm{T}} \mathbf{X}+\Lambda\right)^{-1} \mathbf{X}^{\mathrm{T}} \mathbf{Y}
$$

onde $\Lambda=\operatorname{diag}\left\{0, \mathbf{0}_{\mathrm{h}}, \mathbf{0}_{\mathrm{d}_{1}}, \lambda_{1}^{2 \mathrm{~d}_{\mathrm{d}}} \mathbf{1}_{\mathrm{K}_{1}}, \cdots, \mathbf{0}_{\mathrm{d}_{\mathrm{p}}}, \lambda_{\mathrm{p}}^{2 \mathrm{~d}_{\mathrm{p}}} \mathbf{1}_{\mathrm{K}_{\mathrm{p}}}, \mathbf{0}_{\mathrm{d}+1}, \lambda^{2} \mathbf{1}_{\mathrm{K}}\right\}$ é uma matriz diagonal com $\mathbf{0}_{\mathrm{h}}$ sendo um vetor de zeros de dimensão $h, \mathbf{0}_{\mathrm{d}_{r}}$ e $\mathbf{1}_{\mathrm{K}_{\mathrm{f}}}$ é um vetor de zeros e um vetor de elementos unitários de dimensão $d_{r}$ e $K_{r}$ respectivamente, $r=1, \ldots$, p e $\mathbf{1}_{K}$ é um vetor de elementos unitários de dimensão $\mathrm{K}$. Os $\lambda_{\mathrm{r}}$ representam os parâmetros de suavização a serem fixados para o ajuste das $r$ funções não especificadas $f_{r}, r=1, \ldots, p$, enquanto $\lambda$ é o mesmo para a variável defasada.

Os coeficientes estimados pela equação (3.21) fornecem diretamente estimativas para $\alpha$ e $\psi=\left(\psi_{1}, \ldots, \psi_{\mathrm{h}}\right)^{\mathrm{T}}$. As estimativas para $\mathrm{f}_{\mathrm{r}}\left(\mathrm{s}_{\mathrm{rt}}\right), \mathrm{r}=1, \ldots, \mathrm{p}, \mathrm{t}=\mathrm{q}+1$, ..., n e para $\beta_{\mathrm{j}}, \mathrm{j}=0, \ldots$, q são obtidas a partir de $\hat{\gamma}^{*}$ fazendo

$$
\hat{\mathbf{f}}_{\mathrm{r}}\left(\mathrm{s}_{\mathrm{rt}}\right)=\mathbf{X}_{\text {suav., }} \hat{\boldsymbol{\gamma}}_{\text {suav, }, \mathrm{r}}^{*} \text { e } \hat{\boldsymbol{\beta}}=\mathbf{U} \hat{\gamma}_{\text {def. }}^{*},
$$

onde $\hat{\gamma}_{\text {suav, }, \mathrm{r}}^{*}$ e $\hat{\gamma}_{\text {def. }}$ são as partes de (3.21) correspondentes à r-ésima variável suavizada e à variável defasada respectivamente.

Uma convenção comum em modelos aditivos (Hastie e Tibshirani, 1990) é, no lugar da matriz $\mathbf{X}$ dada em (3.15), que pode ser escrita como $\mathbf{X}=\left[\mathbf{1} \mid \mathbf{X}_{\mathrm{u}}\right]$, trabalhar com a matriz de planejamento da forma

$$
\widetilde{\mathbf{X}}=\left[1 \mid \widetilde{\mathbf{X}}_{\mathrm{u}}\right],
$$


onde $\mathbf{1}$ é um vetor com todos os elementos unitários, e $\widetilde{\mathbf{X}}_{\mathrm{u}}=\left(\mathbf{I}-\frac{1}{\mathrm{n}} \mathbf{1 1} \mathbf{1}^{\mathrm{T}}\right) \mathbf{X}_{\mathrm{u}}$, ou seja, as colunas de $\widetilde{\mathbf{X}}_{u}$ são centradas em torno de suas médias. A correspondente partição no vetor de parâmetros $\gamma^{*}$ é

$$
\gamma^{*}=\left[\alpha \mid \gamma_{u}^{*}\right]^{T}
$$

e a matriz $\Lambda$ é escrita como

$$
\Lambda=\operatorname{diag}\left\{0, \Lambda_{u}\right\}
$$

Assim procedendo, o termo constante $\alpha$ é ortogonal ao restante dos coeficientes e a estimação de $\alpha$ pode ser feita de forma independente dos outros coeficientes. Este fato elimina o problema de não identificabilidade devido à presença do intercepto no ajuste dos modelos aditivos (Hastie e Tibshirani, 1990), ou seja, $\left(\widetilde{\mathbf{X}}_{\mathrm{u}}^{\mathrm{T}} \widetilde{\mathbf{X}}_{\mathrm{u}}+\boldsymbol{\Lambda}_{\mathrm{u}}\right)$ ao contrário de $\left(\mathbf{X}^{\mathrm{T}} \mathbf{X}+\boldsymbol{\Lambda}\right)$, é uma matriz não-singular. Os estimadores de mínimos quadrados de $\alpha$ e $\gamma_{\mathrm{u}}^{*}$ são então dados por

$$
\hat{\alpha}=g(\bar{y}) \text { e } \hat{\gamma}_{u}^{*}=\left(\tilde{\mathbf{X}}_{u}^{\mathrm{T}} \tilde{\mathbf{X}}_{u}+\Lambda_{u}\right)^{-1} \tilde{\mathbf{X}}_{u}^{\mathrm{T}} \mathbf{y}
$$

No caso de erros independentes não normais, a estimação dos parâmetros é feita iterativamente por meio do algoritmo de ponderação local penalizado, que é uma versão modificada do algoritmo de ponderação local, apresentado na seção 2.4. Agora não é necessário o passo do retroajuste, o que reduz significativamente o trabalho computacional. O algoritmo de ponderação local penalizado ajusta simultaneamente um P-spline para cada componente não paramétrico do MAG. A solução obtida maximiza a versão penalizada do logaritmo da fünção de verossimilhança 


$$
l\left(\mathbf{y} ; \gamma^{*}\right)-\frac{1}{2} \sum_{\mathrm{r}=1}^{\mathrm{p}} \lambda_{\mathrm{r}} \gamma_{\mathrm{r}}^{* \mathrm{~T}} \Lambda_{\mathrm{r}} \gamma_{\mathrm{r}}^{*}
$$

onde $l\left(\mathbf{y} ; \boldsymbol{\gamma}^{*}\right)$ representa o logaritmo da função de verossimilhança, $\lambda_{\mathrm{r}}$ é o parâmetro de suavização correspondente à r-ésima variável modelada de forma não paramétrica e $\gamma_{\mathrm{r}}^{*}$ é o vetor composto pelos elementos de $\gamma^{*}$ relativos à r-ésima função não especificada $f_{r}, r=1, \ldots, p$.

Fazendo-se $\gamma_{\mathrm{u}}^{*(0)}=\left(\tilde{\mathbf{X}}_{\mathrm{u}}^{\mathrm{T}} \tilde{\mathbf{X}}_{\mathrm{u}}+\boldsymbol{\Lambda}_{\mathrm{u}}\right)^{-1} \widetilde{\mathbf{X}}_{\mathrm{u}}^{\mathrm{T}} \mathbf{y}$ e $\boldsymbol{\alpha}^{(0)}=\mathrm{g}(\overline{\mathrm{y}})$ sendo $\boldsymbol{\alpha}$ um vetor de dimensão n-q cujos elementos são todos iguais a $g(\bar{y})$, o algoritmo consiste em iterar os seguintes passos para $\mathrm{m}=1,2, \ldots$

Passo 1: Para $\mathrm{t}=\mathrm{q}+1, \ldots, \mathrm{n}$, calcular

$$
z_{t}^{(m)}=\eta_{t}^{(m)}+\left(y_{t}-\mu_{t}^{(m)}\right)\left(\frac{\partial \eta_{t}}{\partial \mu_{t}}\right)_{(m)}
$$

onde $\eta_{t}^{(m)}$ é o t-ésimo elemento de $\boldsymbol{\eta}=\boldsymbol{\alpha}^{(\mathrm{m}-1)}+\widetilde{\mathbf{X}}_{\mathrm{u}} \gamma_{\mathrm{u}}^{*(\mathrm{~m}-1)}, \mu_{\mathrm{t}}^{(\mathrm{m})}=\mathrm{g}^{-1}\left(\eta_{\mathrm{t}}^{(\mathrm{m})}\right)$, e

$$
\mathbf{W}^{(\mathrm{m})}=\operatorname{diag}\left\{\mathrm{W}_{\mathrm{q}_{+1}^{(\mathrm{m})}}, \ldots, \mathbf{W}_{\mathrm{n}}^{(\mathrm{m})}\right\} \text {, onde } \mathrm{w}_{\mathrm{t}}^{(\mathrm{m})}=\left[\left(\frac{\partial \mu_{\mathrm{t}}}{\partial \eta_{\mathrm{t}}}\right)^{(\mathrm{m})}\right]^{2}\left[\left(\frac{\partial \mu_{\mathrm{t}}}{\partial \theta_{\mathrm{t}}}\right)^{(\mathrm{m})}\right]^{-1}
$$

\section{Passo 2: Calcular}

$$
\gamma_{u}^{*(m)}=\left(\tilde{\mathbf{X}}_{u}^{T} \mathbf{W}^{(m)} \widetilde{\mathbf{X}}_{\mathrm{u}}+\boldsymbol{\Lambda}_{\mathrm{u}}\right)^{-1} \widetilde{\mathbf{X}}_{\mathrm{u}}^{\mathrm{T}} \mathbf{W}^{(\mathrm{m})}\left(\mathbf{z}^{(\mathrm{m})}-\boldsymbol{\alpha}^{(\mathrm{m}-1)}\right) \text {, onde } \mathbf{z}^{(\mathrm{m})}=\left[\mathrm{z}_{\mathrm{q}+1}^{(\mathrm{m})}, \ldots, \mathrm{z}_{\mathrm{v}}^{(\mathrm{m})}\right]^{\mathrm{T}} \text { é }
$$

o vetor contendo as $\mathrm{n}-\mathrm{q}$ variáveis modificadas calculadas no passo 1 e $\boldsymbol{\alpha}^{(\mathrm{m})}=\overline{\mathbf{z}}^{(\mathrm{m})}=\frac{\sum_{\mathrm{i}=\mathrm{q}+1}^{\mathrm{n}} z_{1}^{(\mathrm{m})}}{\mathrm{n}-\mathrm{q}}$, ou seja a estimativa do intercepto no passo m é uma média das variáveis modificadas $\mathrm{z}_{\mathrm{t}}$. 
Passo 3: Repetir os passos 1 e 2 até que

$$
\frac{\left\|\gamma_{u}^{*(m)}-\gamma_{u}^{*(m-1)}\right\|^{2}}{\left\|\gamma_{u}^{*(m-1)}\right\|^{2}}<\varepsilon
$$

para um valor $\varepsilon>0$ fixado.

O valor estimado para o intercepto é dado por $\hat{\alpha}=\bar{z}=\sum_{t=q+1}^{n} \frac{z_{t}}{n-q}$, onde $z_{t}$ é o valor da variável aleatória modificada no passo de convergência.

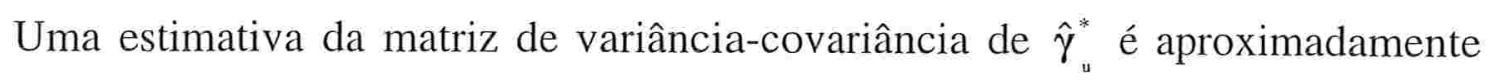
dada por

$$
\operatorname{cov}\left(\hat{\boldsymbol{\gamma}}_{\mathrm{u}}^{*}\right) \cong\left(\tilde{\mathbf{X}}_{\mathrm{u}}^{\mathrm{T}} \hat{\mathbf{W}} \tilde{\mathbf{X}}_{\mathrm{u}}+\boldsymbol{\Lambda}_{\mathrm{u}}\right)^{-1} \tilde{\mathbf{X}}_{\mathrm{u}}^{\mathrm{T}} \hat{\mathbf{W}} \tilde{\mathbf{X}}_{\mathrm{u}}\left(\tilde{\mathbf{X}}_{\mathrm{u}}^{\mathrm{T}} \hat{\mathbf{W}} \tilde{\mathbf{X}}_{\mathrm{u}}+\boldsymbol{\Lambda}_{\mathrm{u}}\right)^{-1}
$$

onde $\hat{\mathbf{W}}=\operatorname{diag}\left\{\mathrm{w}_{\mathrm{q}+1}, \ldots, \mathrm{w}_{\mathrm{n}}\right\}$ contém os pesos na convergência do algoritmo de ponderação local penalizado.

Estimativas para o erros padrão de $\hat{\boldsymbol{\beta}}_{\mathbf{j}}$ e $\hat{\mathbf{f}}_{\mathrm{r}}$ podem ser obtidas de (3.28) e são dadas respectivamente pelos j-ésimos elementos das diagonais das matrizes $\left(\mathbf{U} \operatorname{côv}\left(\hat{\gamma}_{u}^{*}\right)_{\text {def. }} \mathbf{U}^{\mathrm{T}}\right)^{1 / 2}$ e $\left(\mathbf{X}_{\text {suav.,r }} \operatorname{côv}\left(\hat{\gamma}_{\mathrm{u}}^{*}\right)_{\text {suav, }, \mathrm{r}} \mathbf{X}_{\text {suav, }, \mathrm{r}}^{\mathrm{T}}\right)^{1 / 2}$, onde $\mathbf{U}$ é a matriz definida em (3.20), $\operatorname{côv}\left(\hat{\gamma}_{\mathrm{u}}^{*}\right)_{\text {def. }}$ é o bloco de $\operatorname{côv}\left(\hat{\gamma}_{\mathrm{u}}^{*}\right)$ correspondente aos termos defasados, $\mathbf{X}_{\text {suav.r }}$ é como em (3.18) e $\operatorname{côv}\left(\hat{\gamma}_{\mathrm{u}}^{*}\right)_{\text {suav,r, }}$ é o bloco de $\operatorname{côv}\left(\hat{\gamma}_{\mathrm{u}}^{*}\right)$ correspondente aos termos da résima variável modelada de forma não especificada. 
Para a seleção dos parâmetros de suavização pode ser utilizado o critério de Akaike definido em (2.42), isto é,

$$
\mathrm{AIC}=\frac{1}{\mathrm{n}-\mathrm{q}} \mathrm{D}(\mathbf{y} ; \hat{\boldsymbol{\eta}})+\frac{2}{\mathrm{n}-\mathrm{q}} \operatorname{traço}(\mathbf{R}) \phi,
$$

onde $\mathbf{R}=\mathbf{X}\left(\mathbf{X}^{\mathrm{T}} \hat{\mathbf{W}} \mathbf{X}+\boldsymbol{\Lambda}\right)^{-1} \mathbf{X}^{\mathrm{T}} \hat{\mathbf{W}}$.

O critério de validação cruzada (Hastie e Tibshirani, 1990) também pode ser adotado. No caso de erros normais, os valores ajustados para o modelo aditivo com defasagens distribuídas podem ser escritos na forma

$$
\hat{\mathbf{y}}_{\wedge}=\mathbf{G}_{\wedge} \mathbf{y}
$$

onde $\mathbf{G}_{\Lambda}=\frac{1}{n-q} 11^{\mathrm{T}}+\widetilde{\mathbf{X}}_{\mathrm{u}} \hat{\gamma}_{\mathrm{u}}^{*}=\frac{1}{\mathrm{n}-\mathrm{q}} \mathbf{1 1 ^ { \mathrm { T } }}+\widetilde{\mathbf{X}}_{\mathrm{u}}\left(\widetilde{\mathbf{X}}_{\mathrm{u}}^{\mathrm{T}} \widetilde{\mathbf{X}}_{\mathrm{u}}+\boldsymbol{\Lambda}_{\mathrm{u}}\right)^{-1} \widetilde{\mathbf{X}}_{\mathrm{u}}^{\mathrm{T}}$.

O critério de validação cruzada (VC) para selecionar o $\Lambda$ adequado é dado por:

$$
\operatorname{VC}(\Lambda)=\frac{(\mathrm{n}-\mathrm{q})\left\|\left(\mathbf{I}-\mathbf{G}_{\Lambda}\right) \mathbf{y}\right\|^{2}}{\left\{\operatorname{tr}\left(\mathbf{I}-\mathbf{G}_{\Lambda}\right)\right\}^{2}}
$$

Para o caso de erros não normais, $\mathbf{G}_{\wedge}$ é substituído por:

$$
\mathbf{G}_{\wedge}^{\mathrm{w}}=\frac{1}{\mathrm{n}-\mathrm{q}} \mathbf{1 1 ^ { \mathrm { T } }}+\tilde{\mathbf{X}}_{\mathrm{u}}\left(\widetilde{\mathbf{X}}_{\mathrm{u}}^{\mathrm{T}} \hat{\mathbf{W}} \tilde{\mathbf{X}}_{\mathrm{u}}+\boldsymbol{\Lambda}_{\mathrm{u}}\right)^{-1} \tilde{\mathbf{X}}_{\mathrm{u}}^{\mathrm{T}} \hat{\mathbf{W}}
$$

onde $\hat{\mathbf{W}}$ é a matriz diagonal que contém os pesos na convergência do algoritmo de ponderação local. Além disso o vetor y em (3.31) é substituído pelo vetor $\mathbf{z}$ composto pelas variáveis dependentes modificadas do passo 1 deste mesmo algoritmo.

A princípio, os parâmetros de suavização deveriam ser selecionados a partir da minimização do critério de validação cruzada generalizada sobre $\left(\lambda_{1}, \ldots, \lambda_{\mathrm{p}}, \lambda\right)$. Mas 
isto exigiria um extenso trabalho computacional. Na prática, cada parâmetro de suavização é escolhido individualmente, mantendo-se os outros constantes.

Os resultados apresentados neste capítulo serão ilustrados na seção 4.3, na investigação da existência de efeito colheita na mortalidade fetal tardia. 
Capítulo 4

Aplicação

\subsection{Introdução}

A utilização do modelo de defasagens distribuídas é ilustrada neste trabalho considerando dados do estudo realizado por Pereira et al. (1998) no qual foi investigado o efeito da poluição atmosférica na mortalidade de fetos com mais de 28 semanas (mortalidade fetal tardia, ou natimortalidade) na cidade de São Paulo, durante os anos de 1991 e 1992. O banco de dados analisado consistiu de medidas diárias das variáveis confundidoras: temperatura, umidade, dia de observação e dia da semana, das concentrações de poluentes atmosféricos e da variável resposta número de natimortos. Esse banco foi cedido pelo Laboratório de Poluição Atmosférica Experimental da Faculdade de Medicina da USP.

No estudo de Pereira et al. (1998) foi adotado um modelo de regressão de Poisson, com função de ligação logarítmica, sendo consideradas como variáveis preditoras:

- FEV91, MAR91, ..., DEZ92: 23 variáveis indicadoras dos meses, tendo o mês de janeiro de 1991 como referência, que controlam a sazonalidade de longa duração; 
- SEG, ..., SAB: 6 variáveis indicadoras dos dias da semana, tendo domingo como referência, que controlam a sazonalidade de curta duração;

- $\mathrm{M}_{2}$ TEMP: média móvel de 2 dias da temperatura $\left({ }^{\circ} \mathrm{C}\right)$;

- $\mathrm{M}_{2} \mathrm{UMID}$ : média móvel de 2 dias da umidade (\%);

- um controle adicional para temperatura e umidade foi feito categorizando estas variáveis em quatro classes delimitadas pelos seus quartis. Obtiveram-se as classes: "menor que $13,50^{\circ} \mathrm{C}$ ", "de $13,50^{\circ} \mathrm{C}$ a $15,60^{\circ} \mathrm{C}$ ", "de $15,60^{\circ} \mathrm{C}$ a $17,55^{\circ} \mathrm{C}$ " e "maior que $17,55^{\circ} \mathrm{C}$ " para temperatura, e "menor que 59,0\%", "de 59,0\% a 67,5\%", "de 67,5\% a 76,5\%" e "maior que 76,5\%" para umidade relativa. Foram então adicionadas ao modelo variáveis indicadoras dessas categorias (variáveis TEMP1, TEMP2 e TEMP3 para temperatura e UMID1, UMID2 e UMID3 para umidade). As classes "menor que $13,50^{\circ} \mathrm{C}$ "e "menor que 59,0\%" foram consideradas as categorias de referência para temperatura e umidade.

- as concentrações, em $\mu \mathrm{g} / \mathrm{m}^{3}$, da média móvel de ordem 5 do dióxido de nitrogênio $\left(\mathrm{NO}_{2}\right)$, representadas pela variável $\mathrm{M} 5 \mathrm{NO} 2$, foram divididas em 5 intervalos de classes delimitados pelos seus quintis. Quatro variáveis indicadoras (M5NO2a, M5NO2b, M5NO2c e M5NO2d) foram criadas para identificar as 5 categorias, considerando a classe composta pelas menores concentrações como referência.

Pereira et al. (1998) avaliaram também os efeitos dos poluentes dióxido de enxofre $\left(\mathrm{SO}_{2}\right)$, em $\mu \mathrm{g} / \mathrm{m}^{3}$, monóxido de carbono $(\mathrm{CO})$, em ppm, material particulado 
inalável $\left(\mathrm{PM}_{10}\right)$, em $\mu \mathrm{g} / \mathrm{m}^{3}$, e ozônio $\left(\mathrm{O}_{3}\right)$, em $\mu \mathrm{g} / \mathrm{m}^{3}$, sob diferentes defasagens e médias móveis. $\mathrm{O} \mathrm{NO}_{2}$ foi o único que apresentou associação significativa com a natimortalidade. Resultados semelhantes foram obtidos por Lima et al. (2001) que reanalisaram esses mesmos dados por meio de um modelo aditivo generalizado no qual foram consideradas funções não especificadas das mesmas variáveis preditoras contínuas (Hastie e Tibshirani, 1990).

Neste trabalho o efeito do $\mathrm{NO}_{2}$ na natimortalidade é também investigado por meio de modelos aditivos generalizados considerando, porém, concentrações do $\mathrm{NO}_{2}$ em diferentes defasagens em relação à variável resposta. Na seção 4.2 são apresentados resultados obtidos no ajuste de modelos sem e com a restrição paramétrica dada em (2.3), nos quais foram consideradas defasagens de até quatro dias do $\mathrm{NO}_{2}$ em relação à variável resposta. $\mathrm{Na}$ seção 4.3 , o modelo aditivo generalizado com defasagens distribuídas com restrição não paramétrica, descrito no Capítulo 3, foi adotado com o objetivo de avaliar a existência de efeito colheita na natimortalidade considerando defasagens de até trinta dias entre o $\mathrm{NO}_{2}$ e a variável resposta.

O ajuste dos modelos considerados neste capítulo foram realizados por meio de rotinas implementadas no aplicativo S-Plus. A rotina adotada na seção 4.2 foi cedida pelo Laboratório de Poluição Atmosférica Experimental da Faculdade de Medicina da USP. Para o ajuste do modelo adotado na seção 4.3 construímos a rotina apresentada no Apêndice C. 


\subsection{Ajuste de modelos aditivos generalizados com defasagens de até 4 dias entre 0 poluente e a natimortalidade}

Uma análise descritiva pormenorizada dos dados é apresentada em Lima (2001). Reproduzimos na Tabela 4.1 as médias e desvios padrão das variáveis utilizadas neste estudo, a cada ano.

Tabela 4.1: Média ( \pm desvio padrão) para natimortalidade, concentração do $\mathrm{NO}_{2} \mathrm{e}$ variáveis climáticas nos anos de 1991 e 1992.

\begin{tabular}{ccccc}
\hline Variáveis & $\mathrm{n}$ & 1991 & 1992 & Total \\
\hline Variável Resposta & & & & \\
Natimortalidade & 730 & $8,64 \pm 3,11$ & $8,08 \pm 3,04$ & $8,36 \pm 3,08$ \\
\hline Variáveis Climáticas & & & & \\
Umidade & 730 & $67,35 \pm 13,34$ & $69,42 \pm 13,82$ & $68,38 \pm 13,61$ \\
Temperatura & 730 & $15,22 \pm 2,87$ & $15,53 \pm 2,82$ & $15,37 \pm 2,85$ \\
\hline Poluente & & & & \\
$\mathrm{NO}_{2}$ & 690 & $154,75 \pm 78,43$ & $159,05 \pm 84,45$ & $156,82 \pm 81,36$ \\
\hline
\end{tabular}

Podemos observar pela Tabela 4.1, que algumas observações do $\mathrm{NO}_{2}$ não estavam disponíveis no banco de dados original. Um banco completo foi obtido colocando-se no lugar da observação "faltante" a média aritmética da imediatamente anterior com a posterior.

Notamos, na Tabela 4.1, que o número médio de natimortos em 1992 é próximo do observado no ano anterior. O mesmo ocorre com as variáveis climáticas. Já o $\mathrm{NO}_{2}$ apresentou um aumento médio em sua concentração no ano de 1992 em relação ao ano anterior. 
$\mathrm{Na}$ construção dos modelos aditivos generalizados tendo a natimortalidade (NATMOR) como variável resposta foi considerado o mesmo modelo básico descrito em Lima et al. (2001), ou seja,

$$
\begin{gathered}
\ln \left[\mathrm{E}\left(\mathrm{NATMOR}_{\mathrm{t}}\right)\right]=\alpha+\mathrm{f}_{1}(\mathrm{DIA})+\beta_{1} \mathrm{SEG}+\ldots+\beta_{6} \mathrm{SAB}+\beta_{7}\left(\mathrm{M}_{2} \mathrm{TEMP}_{\mathrm{t}}\right)+ \\
\beta_{8}\left(\mathrm{M}_{2} \mathrm{UMID}_{\mathrm{t}}\right),
\end{gathered}
$$

onde

NATIMOR : número de óbitos fetais tardios no dia t;

$\mathrm{f}_{1}$ : função suave não especificada de DIA;

DIA: dia do estudo, variável de controle sazonal de longa duração;

SEG, ..., SAB: 6 variáveis indicadoras para os dias da semana, tendo domingo como referência, que controlam a sazonalidade de curta duração;

$\mathrm{M}_{2} \mathrm{TEMP}_{\mathrm{t}}$ : média móvel de 2 dias da temperatura $\left({ }^{\circ} \mathrm{C}\right)$ no dia $\mathrm{t} \mathrm{e}$ $\mathrm{M}_{2} \mathrm{UMID}_{\mathrm{t}}$ : média móvel de 2 dias da umidade (\%) no dia t.

$\mathrm{O}$ poluente $\mathrm{NO}_{2}$ foi adicionado a (4.1) de diferentes formas, gerando os modelos

- $\ln \left[\mathrm{E}\left(\mathrm{NATMOR}_{\mathrm{t}}\right)\right]=\alpha+\mathrm{f}_{1}(\mathrm{DIA})+\beta_{1} \mathrm{SEG}+\ldots+\beta_{6} \mathrm{SAB}+\beta_{7}\left(\mathrm{M}_{2} \mathrm{TEMP}_{\mathrm{t}}\right)+$

$$
\beta_{8}\left(\mathrm{M}_{2} \mathrm{UMID}_{\mathrm{t}}\right)+\beta_{9} \mathrm{NO}_{2 \mathrm{t}}, \quad \mathrm{t}=1, \ldots, 730,
$$

onde $\mathrm{NO}_{2}$ té a concentração de $\mathrm{NO}_{2}$ no mesmo dia da ocorrência da morte;

- $\quad \ln \left[\mathrm{E}\left(\mathrm{NATMOR}_{\mathrm{t}}\right)\right]=\alpha+\mathrm{f}_{1}(\mathrm{DIA})+\beta_{1} \mathrm{SEG}+\ldots+\beta_{6} \mathrm{SAB}+\beta_{7}\left(\mathrm{M}_{2} \mathrm{TEMP}_{\mathrm{t}}\right)+$

$$
\beta_{8}\left(\mathrm{M}_{2} \mathrm{UMID}_{\mathrm{t}}\right)+\beta_{9}\left(\mathrm{M}^{2} \mathrm{NO}_{2 t}\right), \quad \mathrm{t}=1, \ldots, 730
$$


onde $\mathrm{M}_{5} \mathrm{NO}_{2}$, é a média móvel. de 5 dias das concentrações do $\mathrm{NO}_{2}$ no dia t;

- $\quad \ln \left(\right.$ E $\left.\left._{\text {NATMOR }}\right]\right)=\alpha+f_{1}($ DIA $)+\gamma_{1} \mathrm{SEG}+\ldots+\gamma_{6} \mathrm{SAB}+\gamma_{7}\left(\mathrm{M}_{2} \mathrm{TEMP}_{\mathrm{t}}\right)+$ $\gamma_{8}\left(\mathrm{M}_{2} \mathrm{UMID}_{\mathrm{t}}\right)+\beta_{0} \mathrm{NO}_{2 \mathrm{t}}+\beta_{1} \mathrm{NO}_{2 \mathrm{t}-1}+\ldots+\beta_{4} \mathrm{NO}_{2 \mathrm{t}-4}, \mathrm{t}=5, \ldots, 730,(4.4)$ onde $\mathrm{NO}_{2}$ t-j é a concentração de $\mathrm{NO}_{2} \mathrm{j}$ dias antes da ocorrência da morte, $\mathrm{j}=$ $0, \ldots, 4$, ou seja, um modelo com defasagens distribuídas de até 4 dias, sem restrição nos parâmetros e

- $\left.\quad \ln \left(\mathrm{E}_{\mathrm{NATMOR}}\right]\right)=\alpha+\mathrm{f}_{1}\left(\mathrm{DIA}_{\mathrm{t}}\right)+\gamma_{1} \mathrm{SEG}+\ldots+\gamma_{6} \mathrm{SAB}+\gamma_{7}\left(\mathrm{M}_{2} \mathrm{TEMP}_{\mathrm{t}}\right)+$ $\gamma_{8}\left(\mathrm{M}_{2} \mathrm{UMID}_{\mathrm{t}}\right)+\eta_{0} \mathrm{Z}_{\mathrm{t} 0}+\eta_{1} \mathrm{Z}_{\mathrm{t} 1}+\ldots+\eta_{\mathrm{d}} \mathrm{Z}_{\mathrm{td}}, \mathrm{t}=5, \ldots, 730$, (4.5) onde $Z_{\mathrm{tk}}$ é como em (2.7). Ou seja, (4.5) é um modelo aditivo generalizado com defasagens distribuídas com a restrição polinomial nos parâmetros.

Na escolha da defasagem do poluente nos modelos (4.4) e (4.5) adotamos $q=4$, de forma a obter resultados comparáveis ao modelo (4.3) no qual foi considerada a média móvel de 5 dias do $\mathrm{NO}_{2}$, também adotada nos estudos de Pereira et al. (1998) e Lima (2001).

Schwartz (2000-a) propôs escrever o modelo (4.4) da seguinte forma:

$\ln \left(E_{\left[Y_{t}\right]}\right)=\alpha+f_{1}(D I A)+\gamma_{1} S E G+\ldots+\gamma_{6} S A B+\gamma_{7}\left(M_{2}\right.$ TEMP $\left._{t}\right)+\gamma_{8}\left(M_{2}\right.$ UMID $\left._{t}\right)+$

$$
\beta^{*}\left(\omega_{0} \mathrm{NO}_{2 \mathrm{t}}+\omega_{1} \mathrm{NO}_{2 \mathrm{t}-1}+\ldots+\omega_{4} \mathrm{NO}_{2 \mathrm{t}-4}\right), \quad \mathrm{t}=5, \ldots, 730
$$

onde 


$$
\begin{gathered}
\omega_{i}=\frac{\beta_{i}}{\beta^{*}} \quad i=0, \ldots, 4 \text { e } \\
\beta^{*}=\beta_{0}+\ldots+\beta_{4} .
\end{gathered}
$$

O termo $\left(\omega_{0} \mathrm{NO}_{2 \mathrm{t}}+\omega_{1} \mathrm{NO}_{2 \mathrm{t}-1}+\ldots+\omega_{4} \mathrm{NO}_{2 \mathrm{t}-4}\right)$ em (4.6) é uma média ponderada das concentrações do poluente $\mathrm{NO}_{2}$ defasadas em até 4 unidades de tempo, e $\beta^{*}$ representa o efeito marginal no logaritmo do valor esperado da natimortalidade quando esta média ponderada aumenta em uma unidade. No modelo (4.5) também é possível a construção de $\beta^{*}$, considerando que cada $\beta_{\mathrm{j}}$ é obtido por meio de (2.3).

Os modelos (4.2) a (4.6) possuem apenas um termo não paramétrico, $\mathrm{f}_{1}$ (DIA), e, portanto, no seu processo de ajuste não é utilizado o procedimento de retroajuste (ver Lima et al. (2001), por exemplo). A escolha do suavizador a ser utilizado torna-se, então, menos importante. Por sua simplicidade computacional e também para efeito comparativo com outros estudos, o suavizador loess (Cleveland, 1979) foi utilizado no ajuste desses modelos. A estratégia adotada no ajuste é a mesma descrita, entre outos, em Lima et al.(2001) ou Singer et al.(2002): foi inicialmente ajustado o modelo básico (4.1) e, após essa etapa, passamos ao ajuste dos modelos com o poluente. O parâmetro de suavização adotado para o ajuste de $f_{1}$ foi o mesmo selecionado por Lima et al. (2001), ou seja, $\lambda_{1}=0,5$.

Na Tabela 4.2 são apresentadas as estimativas dos coeficientes do poluente $\mathrm{NO}_{2}$ e seus respectivos erros padrão estimados nos modelos (4.2) e (4.3). Também são apresentados na tabela o nível descritivo para testar a hipótese $H_{0}: \beta_{9}=0$, além da estatística AIC. Observa-se que o efeito da média móvel de 5 dias do $\mathrm{NO}_{2}$ na 
mortalidade é significativo, enquanto que a medida do dia tem efeito marginal, confirmando a existência da defasagem no efeito do poluente $\mathrm{NO}_{2}$ sobre o número médio de natimortos na cidade de São Paulo.

Na Tabela 4.3 são apresentadas estimativas dos coeficientes das variáveis relacionadas ao poluente nas diferentes defasagens nos modelos (4.4) e (4.5), e erros padrão estimados, adotando $\mathrm{d}=2$ como grau do polinômio para o modelo restrito. Também é mostrado o valor da estatística AIC para os modelos sem e com a restrição. A escolha de $\mathrm{d}=2$ como grau do polinômio deveu-se ao fato de que entre os diversos modelos com diferentes graus de polinômios ajustados, o de grau $\mathrm{d}=2$ apresentou um menor valor da estatística AIC. Também são apresentadas nessa tabela estimativas de $\beta^{*}$ e seus respectivos erros padrão estimados para os modelos restrito e irrestrito. Podemos notar que as estimativas obtidas para os coeficientes do poluente no mesmo dia da ocorrência da natimortalidade adotando os modelos com defasagens distribuídas são bastante semelhantes à obtida no ajuste do modelo (4.2) (Tabela 4.2). Observamos também que as estimativas dos erros padrão dos estimadores dos coeficientes do $\mathrm{NO}_{2}$ no modelo restrito são menores que no modelo irrestrito, ou seja, impondo a restrição obtivemos estimativas mais precisas dos coeficientes do poluente. Pode-se também perceber que as estimativas de $\beta^{*}$ e de seu erro padrão para os modelos restritos e irrestritos foram bastante próximas.

Como ilustração, são apresentados no Apêndice A os resultados obtidos quando são considerados outros períodos de defasagem. 
Tabela 4.2: Estimativas dos coeficientes do poluente e erros padrão, níveis descritivos e valores observados do AIC para os modelos (4.2) e (4.3)

\begin{tabular}{lcccc}
\hline Concentração de $\mathrm{NO}_{2}$ & Estimativa & Erro Padrão & Nível Descritivo & AIC \\
\hline Do dia & 0,000257 & 0,000184 & 0,0818 & 514,975 \\
média móvel de 5 dias & 0,000555 & 0,000271 & 0,0202 & 513,515 \\
\hline
\end{tabular}

Tabela 4.3: Estimativas dos coeficientes do poluente e erros padrão considerando concentrações do $\mathrm{NO}_{2}$ defasadas de até 4 dias em relação ao evento de natimortalidade e valores observados do AIC nos modelos irrestrito e restrito $(\mathrm{d}=2)$.

\begin{tabular}{cccc}
\hline Parâmetro & Restrição & Estimativa & Erro Padrão \\
\hline \multirow{2}{*}{$\beta_{0}$} & Não & 0,000232 & 0,000255 \\
& Sim & 0,000199 & 0,000221 \\
\hline \multirow{2}{*}{$\beta_{1}$} & Não & 0,000176 & 0,000268 \\
& Sim & 0,000224 & 0,000115 \\
\hline \multirow{2}{*}{$\beta_{2}$} & Não & 0,000170 & 0,000263 \\
& Sim & 0,000188 & 0,000142 \\
\hline \multirow{2}{*}{$\beta_{3}$} & Não & 0,000168 & 0,000272 \\
& Sim & 0,000092 & 0,000109 \\
\hline \multirow{2}{*}{$\beta_{4}$} & Não & $-0,000112$ & 0,000246 \\
\multirow{2}{*}{$\beta^{*}=\sum_{\mathrm{j}=0}^{4} \beta_{j}$} & Sim & $-0,000065$ & 0,000205 \\
\hline \multicolumn{5}{c}{ Não } & 0,000633 & 0,000326 \\
Modelo Irrestrito: & Sim & 0,000636 & 0,000326 \\
\hline
\end{tabular}

Nas Figuras 4.1 e 4.2 representamos as estimativas por ponto e intervalo dos coeficientes do $\mathrm{NO}_{2}$ obtidas a partir do ajuste dos modelos (4.4) e (4.5), respectivamente. Os intervalos de confiança foram construídos com coeficiente de confiança aproximado de $90 \%$. Por meio desses gráficos podemos visualizar a distribuição do efeito do $\mathrm{NO}_{2}$ na natimortalidade no decorrer do tempo. 


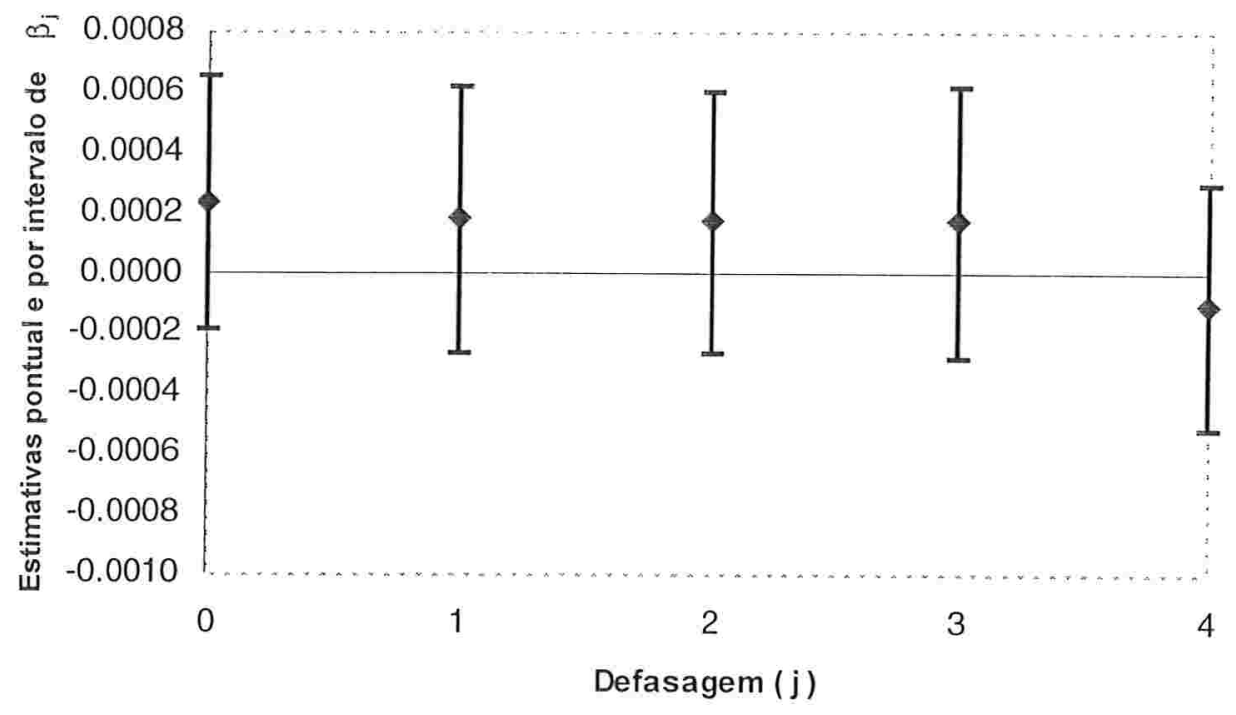

Figura 4.1: Estimativas pontual e por intervalo $(\gamma=0,90)$ dos coeficientes do $\mathrm{NO}_{2}$ considerando defasagens de até 4 dias em relação à ocorrência da natimortalidade para o modelo irrestrito.

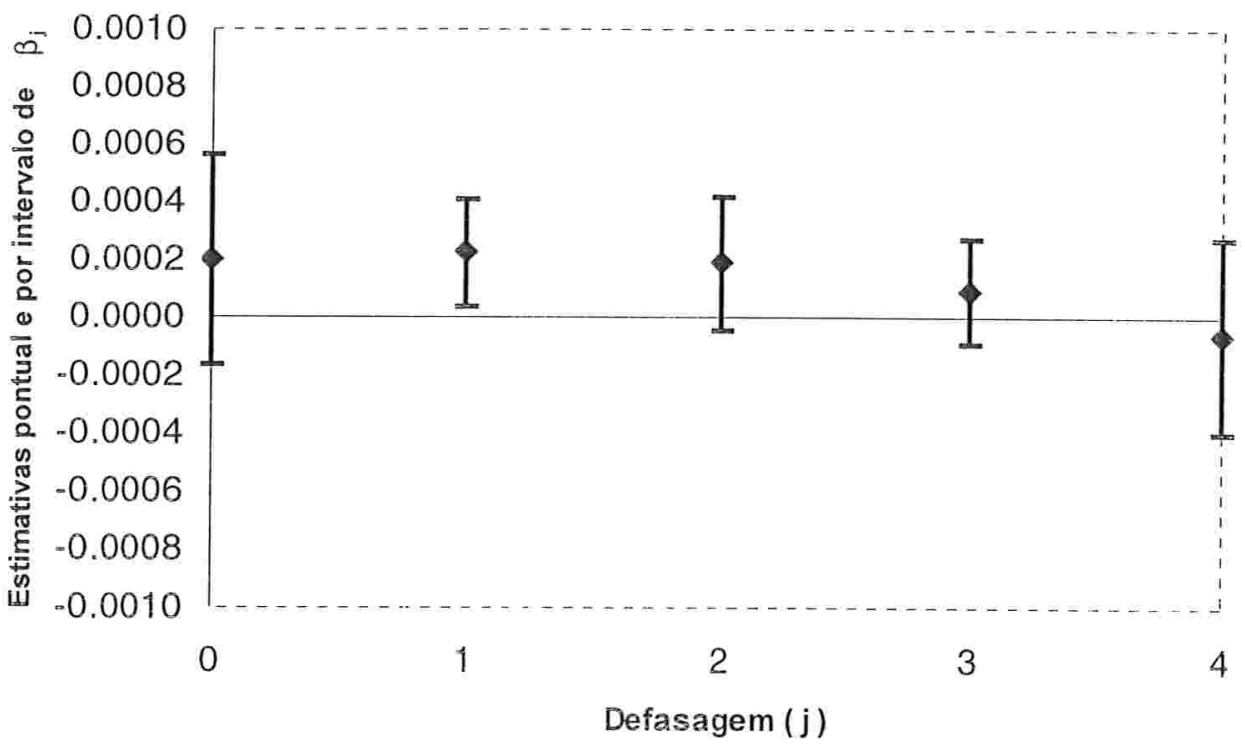

Figura 4.2: Estimativas pontual e por intervalo $(\gamma=0,90)$ dos coeficientes do $\mathrm{NO}_{2}$ considerando defasagens de até 4 dias em relação à ocorrência da natimortalidade para o modelo restrito $(\mathrm{d}=2)$. 
Na Figura (4.1) observamos que o efeito do $\mathrm{NO}_{2}$ na natimortalidade não é significativo qualquer que seja a defasagem. Já no modelo restrito (Figura 4.2), apenas a concentração do dia anterior ao evento de natimortalidade tem efeito significativo.

Considerando outros períodos de defasagem (ver Apêndice A), temos que nos modelos restritos de até 5,6 ou 7 dias de defasagem, é também observado efeito significativo da concentração do poluente em 2 dias anteriores à ocorrência de natimortalidade (Figuras A.2 e A.4, respectivamente).

Um parâmetro bastante utilizado na área de Epidemiologia Ambiental para quantificar o efeito de um poluente em eventos relacionados à natimortalidade ou mobirdade, é o risco relativo (RR) para um aumento de um intervalo interquartil na concentração do poluente. Esse parâmetro é definido como o quociente entre o valor esperado da variável resposta (Y) quando a concentração do poluente assume valor igual a seu terceiro quartil $\left(\mathrm{Q}_{3}\right)$ e o valor esperado de $\mathrm{Y}$ quando a concentração é igual ao seu primeiro quartil $\left(Q_{1}\right)$, mantidos constantes os valores das demais variáveis no modelo. Assim

$$
R R=\frac{E\left(Y \mid \text { concentração do poluente }=Q_{3}\right)}{E\left(Y \mid \text { concentração do poluente }=Q_{1}\right)}
$$

Na Tabela 4.4 são apresentadas as estimativas do risco relativo para um aumento de um intervalo interquartil $\left(93 \mu \mathrm{g} / \mathrm{m}^{3}\right)$ na concentração do $\mathrm{NO}_{2}$ e intervalos de confiança com coeficientes de confiança de 0,95 e 0,90 para o risco relativo nos modelos (4.2) e (4.3). Observamos que o risco de haver mortalidade fetal tardia devido 
ao aumento de $93 \mu \mathrm{g} / \mathrm{m}^{3}$ na média móvel de 5 dias do poluente é $\mathrm{e}^{93 * 0,000555}=1,053$, ou seja, 5,3\% dos natimortos são devido ao aumento da concentração do $\mathrm{NO}_{2}$. Um intervalo de confiança de aproximadamente $95 \%$ para o risco é dado por $\exp [93 *(0,000555 \pm 1,96 * 0,000271)]=[1,002 ; 1,106],($ ver David, 2003$)$

Na Tabela 4.5 podemos ver que os riscos relativos dos modelos com e sem restrição só foram significativos a um coeficiente de confiança de $\gamma=0,90$. As estimativas do risco relativo nos dois modelos (restrito e irrestrito) indicam que um aumento de $93 \mu \mathrm{g} / \mathrm{m}^{3}$ na média ponderada do $\mathrm{NO}_{2}$ no período de zero a quatro dias antes de um determinado dia é responsável por 6,1\% das mortes dos fetos ocorridas nesse dia na cidade de São Paulo. Esta estimativa é maior do que a obtida quando é considerada a média móvel de cinco dias do $\mathrm{NO}_{2}$ (modelo 4.3).

Tabela 4.4: Estimativas do risco relativo para o intervalo interquartil do poluente (RR) e intervalos de confiança para RR (IC) com diferentes coeficientes de confiança para os modelos (4.2) e (4.3).

\begin{tabular}{lccc}
\hline \multicolumn{1}{c}{ Concentração de $\mathrm{NO}_{2}$} & $\mathrm{RR}$ & $\mathrm{IC}(\gamma=0,95)$ & $\mathrm{IC}(\gamma=0,90)$ \\
\hline do dia & 1,024 & {$[0,990 ; 1,059]$} & {$[0,996 ; 1,053]$} \\
média móvel de 5 dias & 1,053 & {$[1,002 ; 1,106]$} & {$[1,010 ; 1,098]$} \\
\hline
\end{tabular}

Tabela 4.5: Estimativas do risco relativo para o intervalo interquartil do poluente (RR) e intervalos de confiança para RR (IC) com diferentes coeficientes de confiança considerando modelos com e sem a restrição polinomial com defasagens de até 4 dias do poluente em relação à ocorrência do evento de natimortalidade.

\begin{tabular}{cccc}
\hline Restrição & RR & IC $(\gamma=0,95)$ & IC $(\gamma=0,90)$ \\
\hline Não & 1,061 & {$[0,999 ; 1,126]$} & {$[1,009 ; 1,115]$} \\
Sim & 1,061 & {$[1,000 ; 1,126]$} & {$[1,009 ; 1,115]$} \\
\hline
\end{tabular}




\subsection{Análise do efeito colheita}

O modelo dado em (3.13) foi adotado na investigação da ocorrência do efeito colheita na natimortalidade. As variáveis confundidoras consideradas foram as mesmas da seção anterior. Por sugestão de especialistas na área de Epidemiologia Ambiental, foram consideradas no modelo defasagens de até 30 dias do $\mathrm{NO}_{2}$ para verificação da existência do efeito colheita. Assim, o modelo a ser ajustado, que é um caso particular do dado em (3.13), é

$$
\begin{aligned}
\ln [\mathrm{E}(\mathrm{NATMOR})]= & \alpha+\psi_{1} \mathrm{SEG}+\ldots+\psi_{6} \mathrm{SAB}+\psi_{7} \mathrm{M}_{2} \mathrm{TEMP}+\psi_{8} \mathrm{M}_{2} \text { UMID } \\
& +\mathrm{f}(\mathrm{DIA})+\sum_{\mathrm{j}=0}^{30} \beta_{\mathrm{j}} \mathrm{NO}_{2 \mathrm{t}-\mathrm{j}}, \quad \mathrm{t}=31, \ldots, 730 .
\end{aligned}
$$

A estratégia adotada foi a mesma citada na seção anterior. No ajuste do modelo básico, o primeiro passo foi modelar a sazonalidade de longa duração, isto é, a variação da natimortalidade em função dos dias de observação, sendo, para isto, considerado o seguinte modelo

$$
\ln \left[\mathrm{E}\left(\mathrm{NATMOR}_{\mathrm{t}}\right)\right]=\alpha+\mathrm{f}(\mathrm{DIA}), \quad \mathrm{t}=31, \ldots, 730
$$

No ajuste de (4.10) foi utilizado o suavizador $P$-spline fixando-se 23 nós, um para cada mudança de mês, conforme feito em Zanobetti et al. (2000). O parâmetro de suavização $\lambda_{1}$ foi escolhido procurando tornar a soma das autocorrelações dos resíduos a mais próxima possível de zero, procedimento que é usual na área de aplicação (Zanobetti et al., 2000). O grau do polinômio adotado neste ajuste foi $\mathrm{d}_{1}=3$, por este apresentar um menor valor da soma de autocorrelação parcial dos resíduos do 
desvio em relação ao polinômio de segundo grau. Alguns valores de $\lambda_{1}$ considerados, correspondentes somas de autocorrelação dos resíduos, e gráficos da função de autocorrelação parcial são apresentados na Figura 4.3.

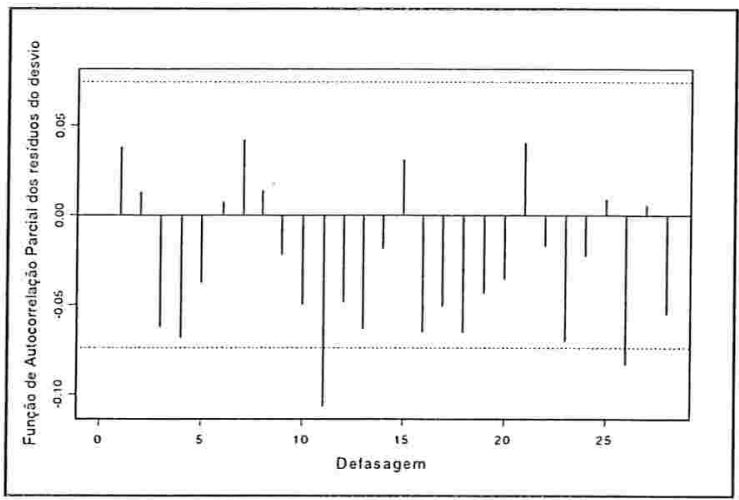

$\lambda_{1}=50$ Soma $=-0,786$

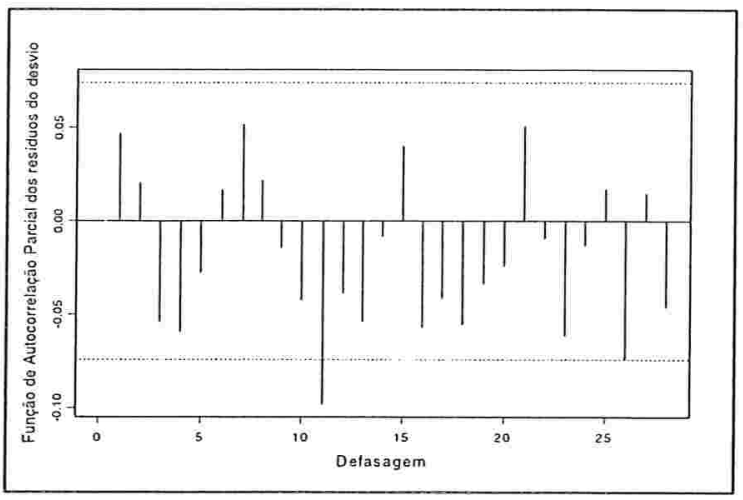

$\lambda_{1}=150$ Soma $=-0,530$

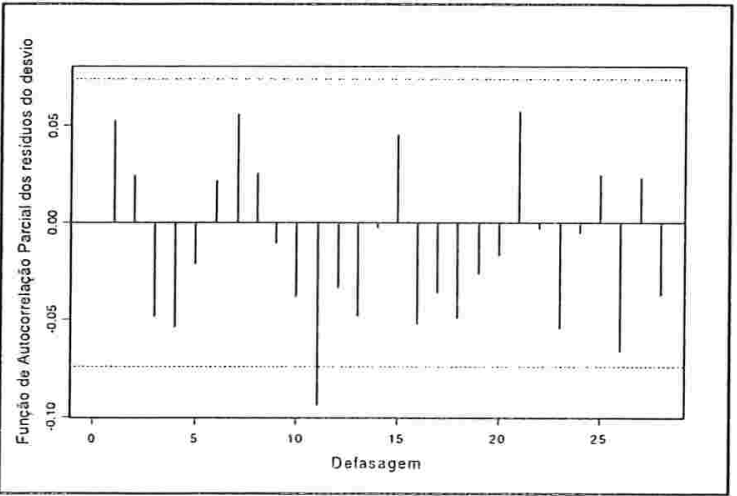

$\lambda_{1}=250$ Soma $=-0,367$

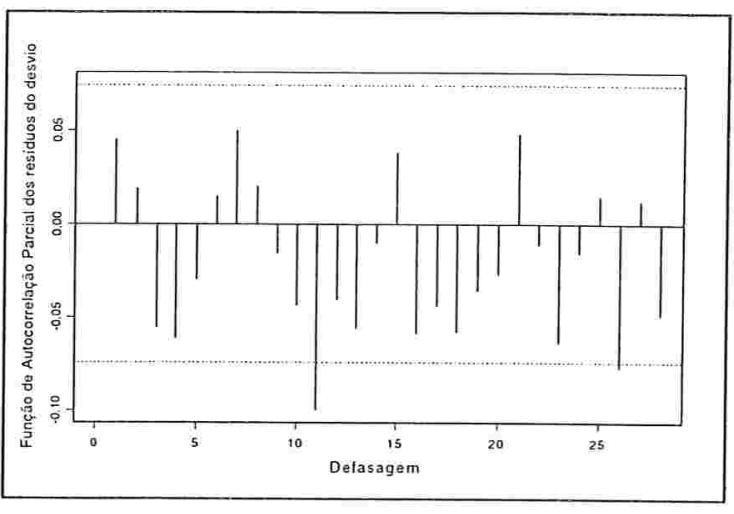

$\lambda_{1}=100$ Soma $=-0,585$

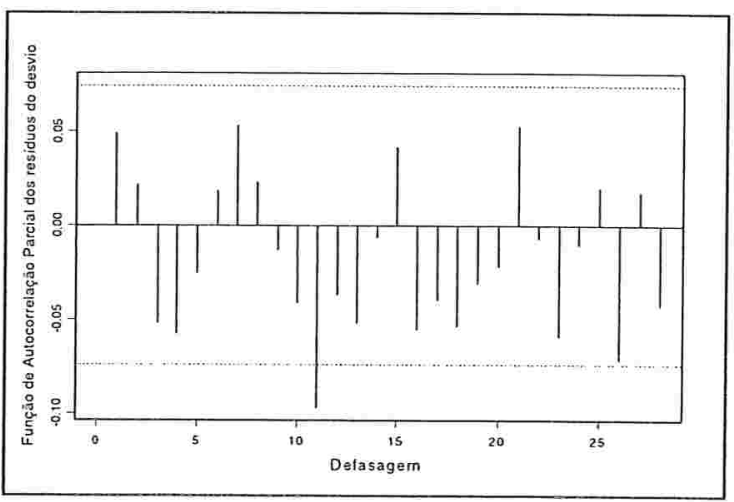

$\lambda_{1}=200$ Soma $=-0,475$

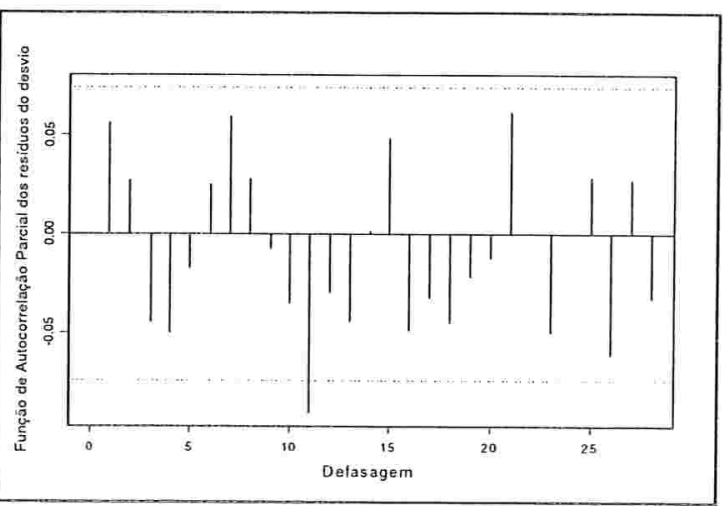

$\lambda_{1}=300$ Soma $=-0,261$

Figura 4.3: Gráficos da função de autocorrelação parcial e valores das somas das autocorrelações parciais dos resíduos do desvio para os diversos valores do parâmetro de suavização $\lambda_{1}$. 


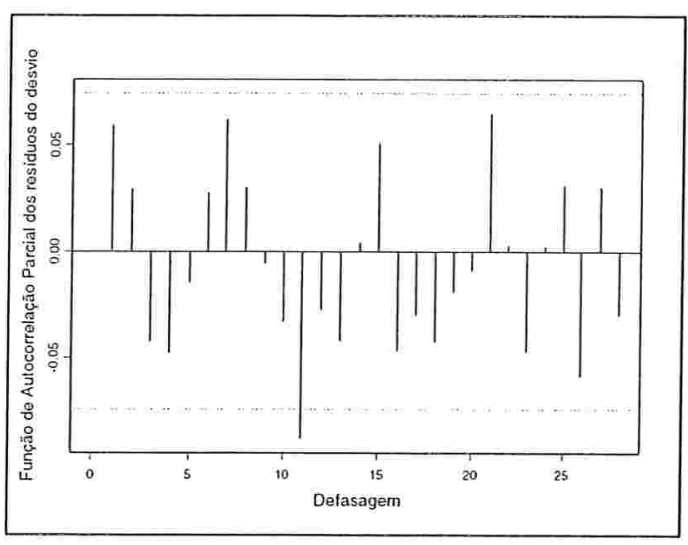

$\lambda_{1}=350$ Soma $=-0,190$

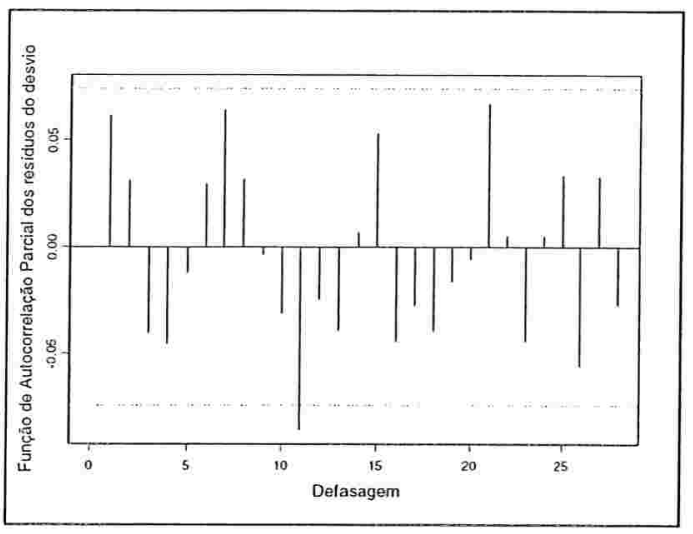

$\lambda_{1}=450$ Soma $=-0,118$

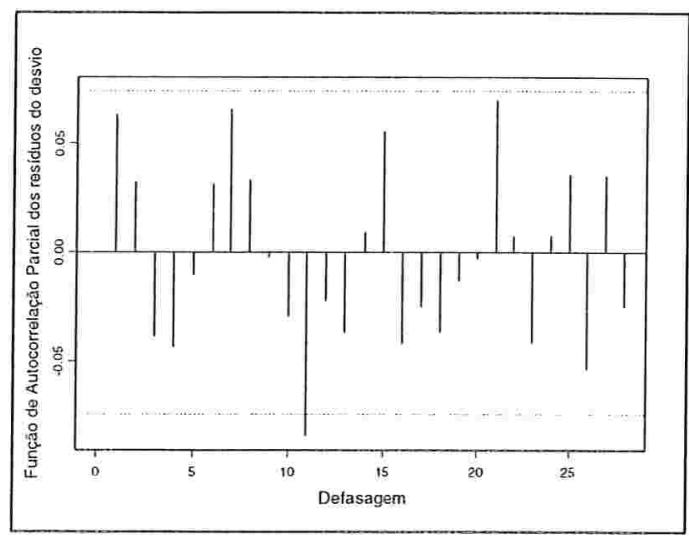

$\lambda_{1}=550$ Soma $=-0,063$

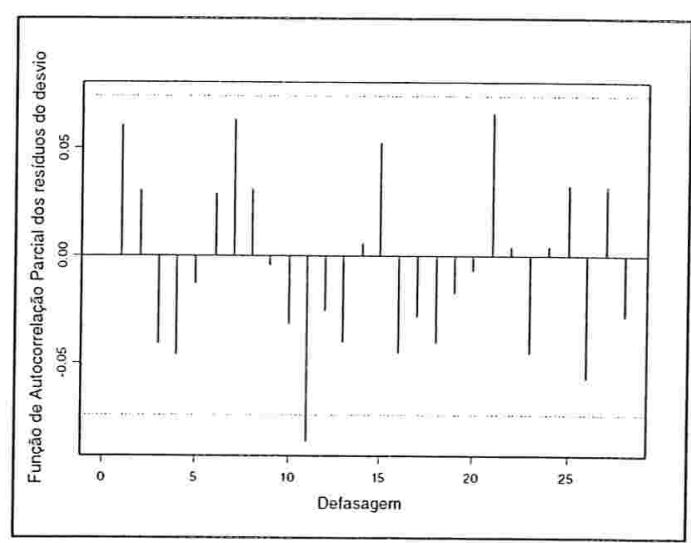

$\lambda_{1}=400$ Soma $=-0,147$

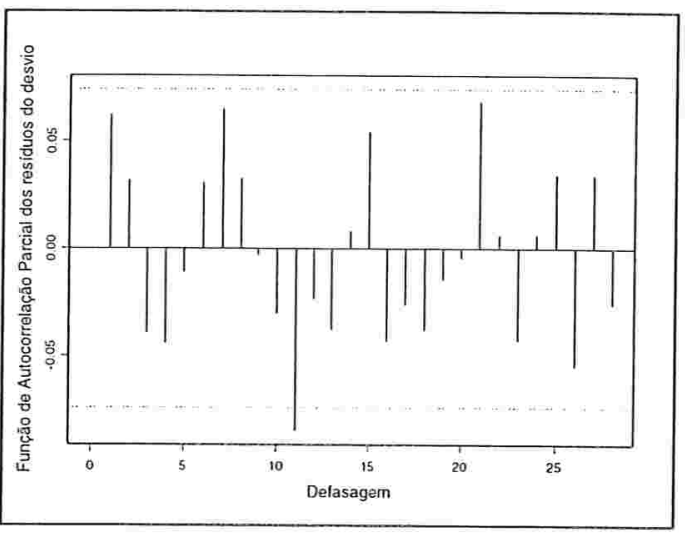

$\lambda_{1}=500$ Soma $=-0,092$

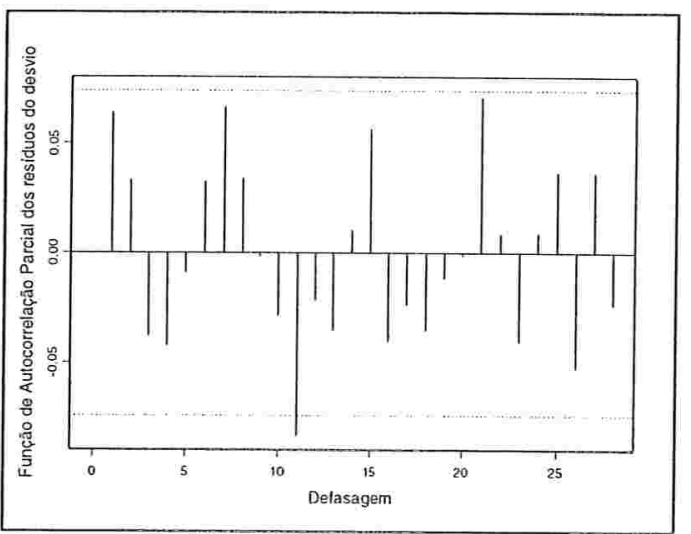

$\lambda_{1}=600$ Soma $=-0,032$

Figura 4.3: Continuação 
Embora o critério adotado para a escolha do parâmetro de suavização fosse através da minimização da soma da autocorrelação parcial dos resíduos, na prática, como pode ser observado na Figura 4.3, isto acarretaria na escolha de um valor muito alto para $\lambda_{1}$ que tornaria a curva muito suavizada impedindo a visualização do efeito colheita. Por este motivo, e também pela semelhança entre os gráficos apresentados, resolvemos adotar $\lambda_{1}=500$ como o parâmetro de suavização.

Após estabelecido o valor do parâmetro $\lambda_{1}$, acrescentamos ao modelo (4.10) as demais variáveis preditoras.

A restrição (3.7) foi imposta aos coeficientes $\beta_{\mathrm{j}}, \mathrm{j}=0, \ldots, 30$, sendo adotada uma sequência de nós igualmente espaçados dados pelos seguintes valores de defasagem: $5,10,15,20,25$ e um polinômio de grau $d=2$. A escolha desse valor como grau do polinômio deve-se ao fato deste ter apresentado um menor valor da estatística AIC para diversos valores pré-fixados de $\lambda$, em relação ao polinômio de grau 3.

O próximo passo foi escolher o parâmetro de suavização a ser adotado na restrição. Para isso, foram ajustados diversos modelos com diferentes valores do parâmetro de suavização $\lambda$, tendo sido escolhido aquele que minimizou o critério de validação cruzada descrito em (3.32). Na Tabela 4.6 são apresentados o desvio, número de graus de liberdade e valores da estatística VC para diferentes escolhas do parâmetro de suavização $\lambda$. Conforme pode ser visto nessa tabela, o valor de $\lambda$ que minimizou o critério de validação cruzada foi $\lambda=4,213 * 10^{5}$. Para valores de $\lambda$ abaixo de $10^{3}$ e acima de $10^{8}$ ocorre uma estabilização das estatísticas mostradas na tabela. O 
desvio do modelo foi de 709,2 com 678,5 graus de liberdade, o que sugere um bom ajuste do modelo. Na próxima seção mostraremos alguns gráficos relacionados a técnicas de diagnóstico com o objetivo de avaliar o ajuste do modelo.

Tabela 4.6: Valores observados do desvio, graus de liberdade (gl) e critério de validação cruzada (VC) correspondentes a diferentes parâmetros de suavização $\lambda$ fixados, utilizando um polinômio de grau $\mathrm{d}=2$ na restrição do poluente.

\begin{tabular}{|c|c|c|c|}
\hline \multicolumn{4}{|c|}{$\begin{array}{c}\text { Modelo } \mathrm{q}=30 \mathrm{~d}_{1}=3 \quad \mathrm{~d}=2 \lambda_{1}=500 \\
\delta=(5,10,15,20,25)\end{array}$} \\
\hline$\lambda$ & Desvio & gl & $\mathrm{VC}$ \\
\hline $10^{3}$ & 707,8 & 675,0 & 1,085 \\
\hline $10^{4}$ & 707,8 & 675,0 & 1,084 \\
\hline $10^{5}$ & 708,6 & 676,8 & 1,078 \\
\hline $4,213 * 10^{5}$ & 709,2 & 678,5 & 1,074 \\
\hline $10^{6}$ & 710,9 & 679,3 & 1,075 \\
\hline $10^{7}$ & 713,7 & 680,0 & 1,078 \\
\hline $10^{8}$ & 713,8 & 680,0 & 1,078 \\
\hline
\end{tabular}

Na Figura 4.4 são representadas as estimativas por ponto e intervalo de $\beta_{\mathrm{j}}, \mathrm{j}=$ 0..., 30, obtidas no ajuste do modelo (4.9). Nessa figura são identificadas as regiões A, $\mathrm{B}$ e $\mathrm{C}$ correspondentes às ilustradas na Figura 1.2. O comportamento observado das estimativas dos coeficientes $\beta_{\mathrm{j}}, \mathrm{j}=0, \ldots, 30$, sugere a existência de efeito colheita do $\mathrm{NO}_{2}$ na natimortalidade. Pode-se perceber que o efeito que $\mathrm{o} \mathrm{NO}_{2}$ exerce na natimortalidade é positivo até os 5 dias anteriores ao evento de morte e do sexto ao décimo nono dia anterior ele é negativo, caracterizando um efeito protetor, decorrente da morte "antecipada" dos indivíduos que pertenciam ao grupo de risco. Após o décimo nono dia o efeito passa a ser positivo novamente até o vigésimo quinto dia. $\mathrm{Na}$ 
Tabela B.1 são apresentadas estimativas e erros padrão para os parâmetros do modelo (4.9).

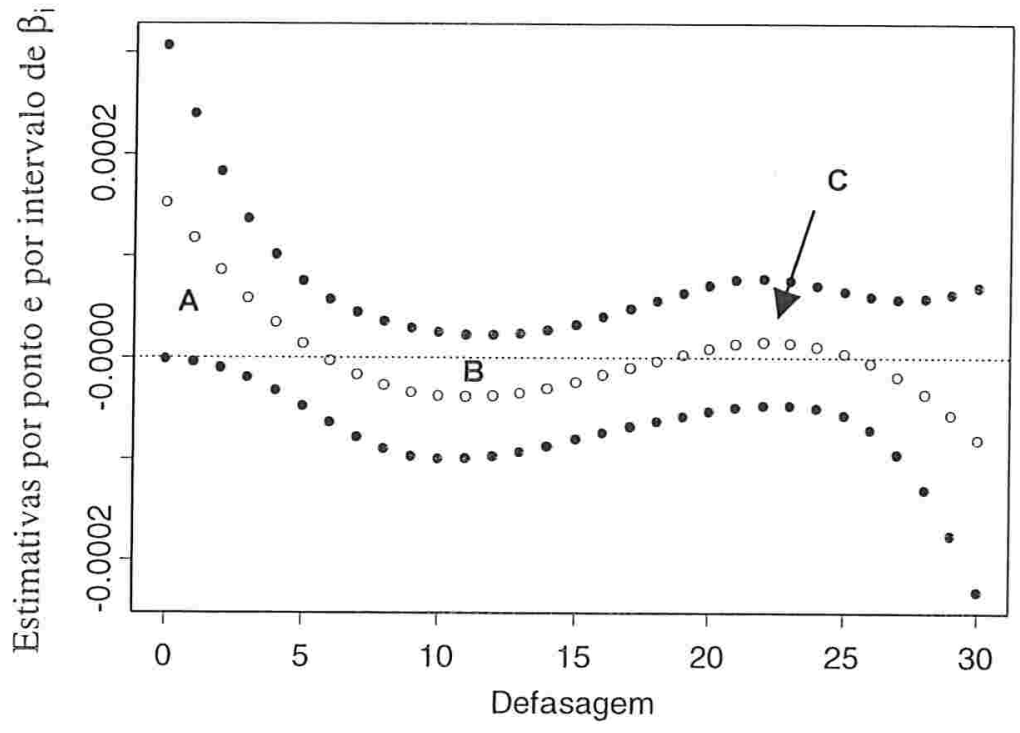

Figura 4.4: Estimativas pontual e por intervalo $(\gamma=0,95)$ dos coeficientes do $\mathrm{NO}_{2}$ no modelo (4.9).

Observamos que o termo referente ao poluente em (4.9), $\sum_{\mathrm{j}=0}^{30} \beta_{\mathrm{j}}\left(\mathrm{NO}_{2}{ }_{\mathrm{t}-\mathrm{j}}\right)$, pode ser definido como:

$\beta^{*}\left(\omega_{0} \mathrm{NO}_{2 \mathrm{t}}+\omega_{1} \mathrm{NO}_{2 \mathrm{t}-1}+\ldots+\omega_{25} \mathrm{NO}_{2 \mathrm{t}-25}\right)+\beta_{26} \mathrm{NO}_{2 \mathrm{t}-26}+\ldots+\beta_{30} \mathrm{NO}_{2 \mathrm{t}-30}, \mathrm{t}=31, \ldots, 730$ onde $\omega_{i}=\frac{\beta_{i}}{\beta^{*}} \quad i=0, \ldots, 25$ e $\beta^{*}=\beta_{0}+\ldots+\beta_{25}$.

Considerando as regiões delimitadas na Figura 4.4 podemos ainda escrever:

$$
\begin{aligned}
\beta^{*}\left(\omega_{0} \mathrm{NO}_{2 \mathrm{t}}+\omega_{1} \mathrm{NO}_{2 \mathrm{t}-1}+\ldots+\omega_{25} \mathrm{NO}_{2 \mathrm{t}-25}\right) & =\beta_{\mathrm{A}}^{*}\left(\omega_{0}^{\prime} \mathrm{NO}_{2 \mathrm{t}}+\ldots+\omega_{5}^{\prime} \mathrm{NO}_{2 \mathrm{t}-5}\right) \\
& +\beta_{\mathrm{B}}^{*}\left(\omega_{6}^{\prime} \mathrm{NO}_{2 \mathrm{t}-6}+\ldots+\omega_{19}^{\prime} \mathrm{NO}_{2 \mathrm{t}-19}\right) \\
& +\beta_{\mathrm{C}}^{*}\left(\omega_{20}^{\prime} \mathrm{NO}_{2 \mathrm{t}-20}+\ldots+\omega_{25}^{\prime} \mathrm{NO}_{2 \mathrm{t}-25}\right)
\end{aligned}
$$


onde $\omega_{i}^{\prime}= \begin{cases}\frac{\beta_{i}}{\beta_{A}^{*}}, & i=0, \ldots, 5 \\ \frac{\beta_{i}}{\beta_{B}^{*}}, & i=6, \ldots, 19 \\ \frac{\beta_{i}}{\beta_{\mathrm{C}}^{*}}, & i=20, \ldots, 25\end{cases}$

ou seja, $\quad \beta_{\mathrm{A}}^{*}=\beta_{0}+\ldots+\beta_{5}, \quad \beta_{\mathrm{B}}^{*}=\beta_{6}+\ldots+\beta_{19}$ e $\beta_{\mathrm{C}}^{*}=\beta_{20}+\ldots+\beta_{25}$ são os efeitos marginais do poluente nas regiões $\mathrm{A}, \mathrm{B}$ e $\mathrm{C}$ respectivamente.

Na Tabela 4.7 são apresentados os efeitos marginais estimados $\hat{\beta}_{A}^{*}, \hat{\beta}_{B}^{*}, \hat{\beta}_{C}^{*}$ e $\hat{\beta}^{*}$, os erros padrão destes efeitos, os riscos relativos (RR) estimados para a variação de um intervalo interquartil da concentração do poluente e os intervalos de confiança aproximados para os $\operatorname{RR}(\gamma=0,95)$.

Tabela 4.7: Estimativas de $\beta^{*}$ e erros padrão estimados de $\beta^{*}(\mathrm{EP})$, riscos relativos para o intervalo interquartil do poluente (RR) e intervalos de confiança para $R R(\gamma=$ 0,95), por região delimitada na Figura 4.4.

\begin{tabular}{ccccc}
\hline Região & $\begin{array}{c}\text { Efeito marginal } \\
\text { estimado }\end{array}$ & EP estimado & RR estimado & Intervalo de Confiança \\
\hline A & 0,000612 & 0,000268 & 1,059 & {$[1,008 ; 1,112]$} \\
B & $-0,000416$ & 0,000331 & 0,962 & {$[0,906 ; 1,022]$} \\
C & 0,000048 & 0,000175 & 1,004 & {$[0,9731,037]$} \\
A+B+C & 0,000244 & 0,000448 & 1,023 & {$[0,9431,110]$} \\
\hline
\end{tabular}

A estimativa de $\beta^{*}$ na região $A$ corresponde à soma dos coeficientes estimados do $\mathrm{NO}_{2}$ nos primeiros 5 dias antecedentes à ocorrência da morte. Portanto, o risco de haver mortalidade fetal tardia devido ao aumento de 93 unidades na média ponderada das concentrações de $\mathrm{NO}_{2}$ durante um período de 6 dias (o atual e mais 5 dias prévios) 
é de 1,059. A mesma interpretação pode ser feita para os riscos relativos das demais regiões. A estimativa de $\beta^{*}$ da região $(A+B+C)$, conforme indicado em (4.7), representa o efeito marginal estimado no logaritmo da natimortalidade quando há um aumento de uma unidade na média ponderada das concentrações do $\mathrm{NO}_{2}$ defasadas de até 25 dias. Vemos, pela Tabela 4.4, que o risco esperado de haver mortalidade fetal tardia neste período devido ao aumento de 93 unidades na média ponderada da concentração do poluente é de 0,935 . O "efeito protetor" desta estimativa ocorreu por causa das estimativas dos coeficientes do poluente que foram negativas na maioria das defasagens (região B), porém esta estimativa não foi significativa conforme podemos observar pelo intervalo de confiança para o risco relativo. Apenas o risco relativo referente à região A foi significativo.

\subsection{Diagnóstico}

As técnicas de diagnóstico são ferramentas utilizadas em modelos de regressão para verificar a adequação do modelo utilizado e a existência ou não de outliers e observações influentes. As técnicas diagnóstico para os modelos aditivos generalizados são bastante similares às técnicas para os modelos lineares generalizados (ver por exemplo, Paula (2002)).

Uma medida informal da qualidade do ajuste de um modelo aditivo generalizado é o desvio do modelo, onde espera-se que valores grandes do desvio quando comparados aos seus graus de liberdade são indicadores de que o modelo 
ajustado não está adequado. Pela Tabela 4.3, um bom ajuste do modelo é sugerido devido à proximidade do desvio em relação aos graus de liberdade.

A análise gráfica dos resíduos do modelo ajustado também é de grande importância entre as técnicas de diagnóstico. Neste trabalho foi feita a análise dos resíduos do desvio que no caso da distribuição de Poisson é dado por:

$$
D_{i}=\operatorname{sinal}\left(y_{i}-\hat{\mu}_{i}\right)\left\{2\left[y_{i} \log \left(y_{i} / \hat{\mu}_{i}\right)-\left(y_{i}-\hat{\mu}_{i}\right)\right]\right\}^{1 / 2} \quad i=1, \ldots, n .
$$

onde $\hat{\mu}_{\mathrm{i}}$ é a estimativa da média da variável resposta $\mathrm{y}_{\mathrm{i}}$ obtida através do ajuste do modelo.

Construímos na Figura 4.5(a), o gráfico dos resíduos do desvio contra índice (i), onde observa-se uma distribuição aleatória dos resíduos em torno do eixo zero. A curva suavizada obtida pelo método loess não sugere nenhuma tendência nos resíduos.

Na Figura 4.5(b), apresentamos o gráfico da função de autocorrelação dos resíduos do desvio, onde são observados valores não significativos das autocorrelações para todas as defasagens maiores que zero.

(a)

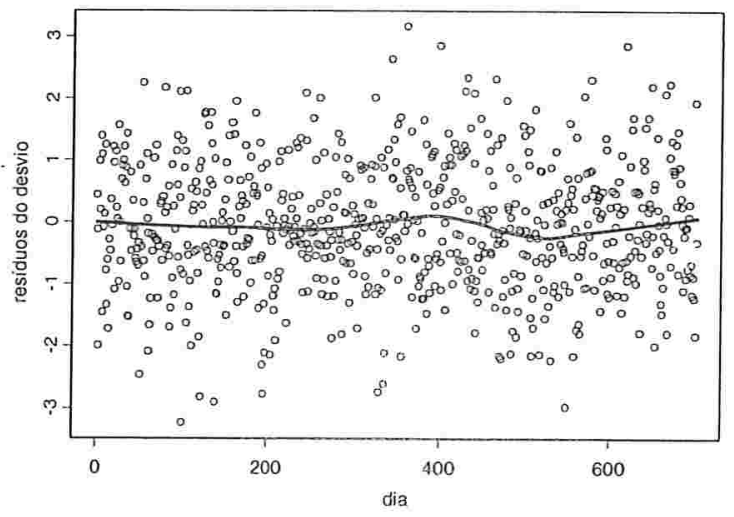

(b)

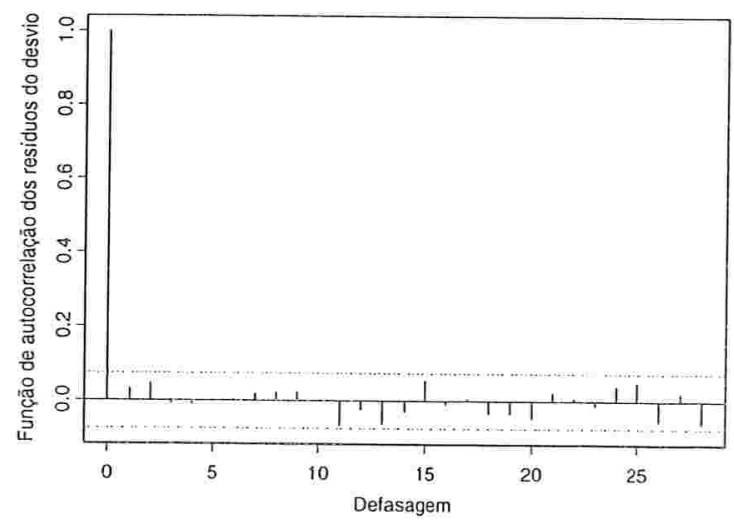

Figura 4.5: Gráfico suavizado dos resíduos do desvio contra índice (a) e gráfico da função de autocorrelação dos resíduos do desvio (b) para o modelo (4.9). 


\subsection{Conclusões}

$\mathrm{Na}$ análise dos modelos aditivos generalizados com curtos períodos de defasagem das concentrações do $\mathrm{NO}_{2}$ em relação à ocorrência de natimortalidade, foi observado que utilizando a restrição polinomial obtemos estimativas mais precisas para os coeficientes do poluente.

Ao utilizarmos defasagens de até 4 dias dos níveis do poluente em relação ao evento de natimortalidade, verificamos que o risco de haver natimortos em decorrência de um aumento de $93 \mu \mathrm{g} / \mathrm{m}^{3}$ na concentração do $\mathrm{NO}_{2}$ foi superior ao risco obtido quando foi utilizado média móvel de 5 dias deste poluente $(6,1 \%$ contra $5,3 \%$ respectivamente). A estimativa do efeito total $\beta^{*}$ e do seu erro padrão praticamente não se alteram quando é imposta a restrição polinomial aos coeficientes do poluente.

Com relação à análise utilizando modelos com longos períodos de defasagem, os resultados obtidos sugerem um efeito colheita no número médio de natimortos provocado pelo aumento da concentração de $\mathrm{NO}_{2}$ no ar na cidade de São Paulo. Observou-se entretanto que ocorreu efeito significativo do $\mathrm{NO}_{2}$ apenas em um curto período de defasagem de até 5 dias antes do evento de natimortalidade. $O$ risco de ocorrência de natimortalidade devido a um aumento de $93 \mu \mathrm{g} / \mathrm{m}^{3}$ na concentração do $\mathrm{NO}_{2}$ nesse período é de $5,9 \%$, bastante próximo ao obtido no ajuste do modelo com defasagens de até 4 dias em relação à ocorrência do evento de natimortalidade.

No capítulo seguinte serão apresentadas algumas considerações finais a respeito deste trabalho. 


\section{Capítulo 5}

\section{Considerações finais}

Neste trabalho apresentamos modelos paramétricos e semi-paramétricos com defasagens distribuídas considerando que a distribuição da variável resposta pertence à família exponencial. No ajuste dos modelos semi-paramétricos, sugerimos que o método de suavização P-spline fosse utilizado, pois desta forma o procedimento do retroajuste pode ser eliminado na estimação dos modelos aditivos generalizados.

Consideramos modelos sem e com restrição nos coeficientes da variável explicativa defasada. Vimos que, quando o período de defasagem é longo, restrições não paramétricas podem ser adotadas, por estabelecerem uma relação menos rígida entre os coeficientes e o tempo de defasagem. O método de suavização $P$-spline foi adotado para estimar essa relação.

Na utilização do $P$-spline foi considerada a base "série de potência truncada" considerada nas seções 3.3 e 3.4. Einbeck (2001) estendeu a teoria de ajuste de polinômios locais, até então restrita a funções de bases polinomiais $\left(1, x, x^{2}, \ldots, x^{d}\right)$, a uma ampla classe de funções de base e discutiu as vantagens e desvantagens da utilização desse procedimento. Em próximos estudos, sugerimos que a utilização de bases mais gerais sejam investigadas em substituição à base "série de potência truncada".

O desenvolvimento deste trabalho foi direcionado de forma a aplicar os modelos com defasagens distribuídas à área de Epidemiologia Ambiental, mais 
especificamente, no estudo dos efeitos da poluição atmosférica sobre a saúde de populações urbanas. Vimos que a introdução no modelo das concentrações do poluente, defasadas em até 30 dias em relação ao dia em que foi observado o evento de mortalidade ou morbidade, permite avaliar a distribuição do efeito da poluição no decorrer do tempo. Nesta área de aplicação, de uma forma geral, é comum a ocorrência de observações aberrantes na variável resposta (mortalidade ou morbidade), ou nas variáveis explicativas (temperatura, umidade, etc.) e outras possíveis confundidoras (Singer et al., 2002). Neste trabalho métodos não robustos de ajuste foram adotados. A utilização de métodos não sensíveis a observações discrepantes na variável resposta e nas confundidoras (Einbeck et al., 2002) devem ser pesquisadas. 


\section{Alguns resultados de ajustes de modelos aditivos generalizados com defasagens de até 7 dias do poluente em relação ao evento de natimortalidade}

Tabela A.1: Estimativas do coeficiente do poluente e erros padrão considerando concentração do $\mathrm{NO}_{2}$ defasadas de até 7 dias em relação ao evento de natimortalidade e valores observados do AIC nos modelos irrestrito e restrito $(d=2)$.

\begin{tabular}{cccc}
\hline Parâmetro & Restrição & Estimativa & Erro Padrão \\
\hline \multirow{2}{*}{$\beta_{0}$} & Não & 0,000242 & 0,000256 \\
& Sim & 0,000191 & 0,000181 \\
\hline \multirow{2}{*}{$\beta_{1}$} & Não & 0,000166 & 0,000269 \\
& Sim & 0,000217 & 0,000094 \\
\hline \multirow{2}{*}{$\beta_{2}$} & Não & 0,000186 & 0,000266 \\
\multirow{2}{*}{$\beta_{3}$} & Sim & 0,000205 & 0,000074 \\
\hline \multirow{2}{*}{$\beta_{4}$} & Não & 0,000202 & 0,000276 \\
& Sim & 0,000157 & 0,000089 \\
\hline \multirow{2}{*}{$\beta_{5}$} & Não & 0,000001 & 0,000277 \\
& Sim & 0,000071 & 0,000090 \\
\hline \multirow{2}{*}{$\beta_{6}$} & Não & 0,000015 & 0,000279 \\
& Sim & $-0,000052$ & 0,000075 \\
\hline \multirow{2}{*}{$\beta_{7}$} & Não & $-0,000199$ & 0,000281 \\
$\beta^{*}=\sum_{\mathrm{j}=0}^{7} \beta_{\mathrm{j}}$ & Sim & $-0,000213$ & 0,000085 \\
\hline & Não & $-0,000447$ & 0,000253 \\
& Nim & $-0,000410$ & 0,000165 \\
\hline Sim & 0,000167 & 0,000373 \\
\hline Modelo Irrestrito: & 520,365 & 0,000166 & 0,000373 \\
\hline
\end{tabular}




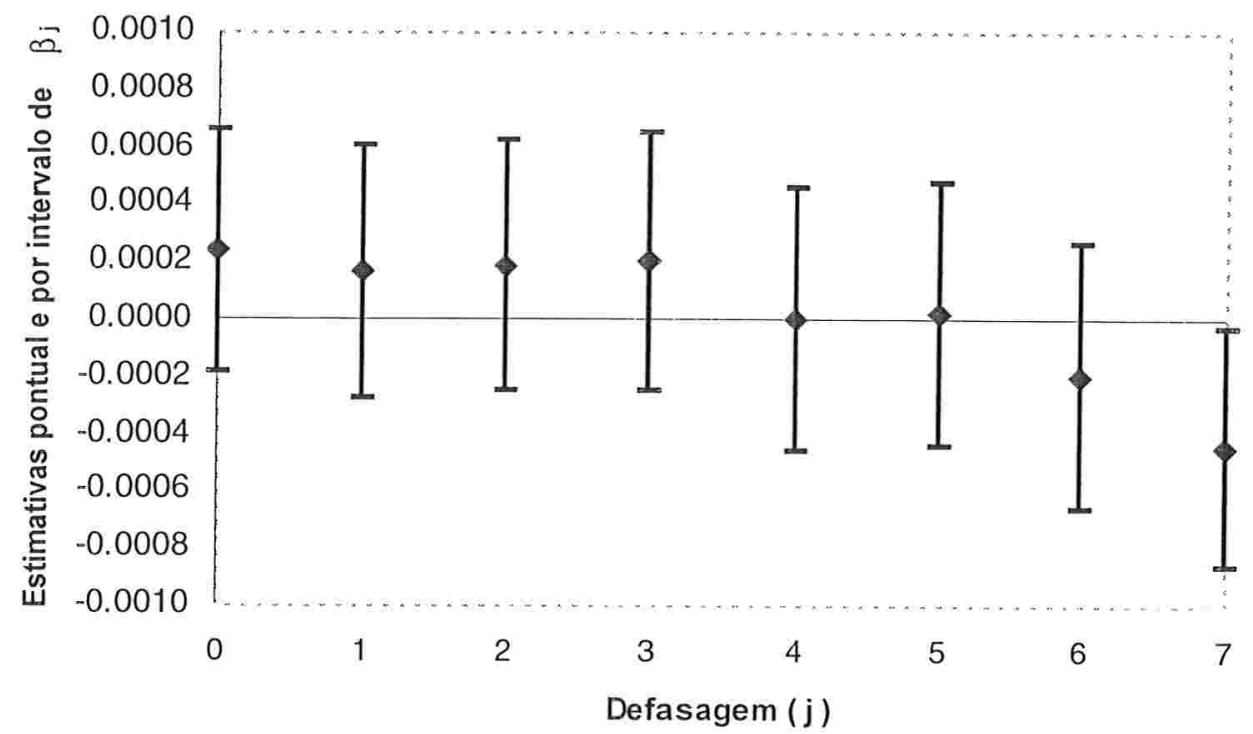

Figura A.1: Estimativas pontual e por intervalo $(\gamma=0,90)$ dos coeficientes do $\mathrm{NO}_{2}$ considerando defasagens de até 7 dias em relação à ocorrência da natimortalidade para o modelo irrestrito.

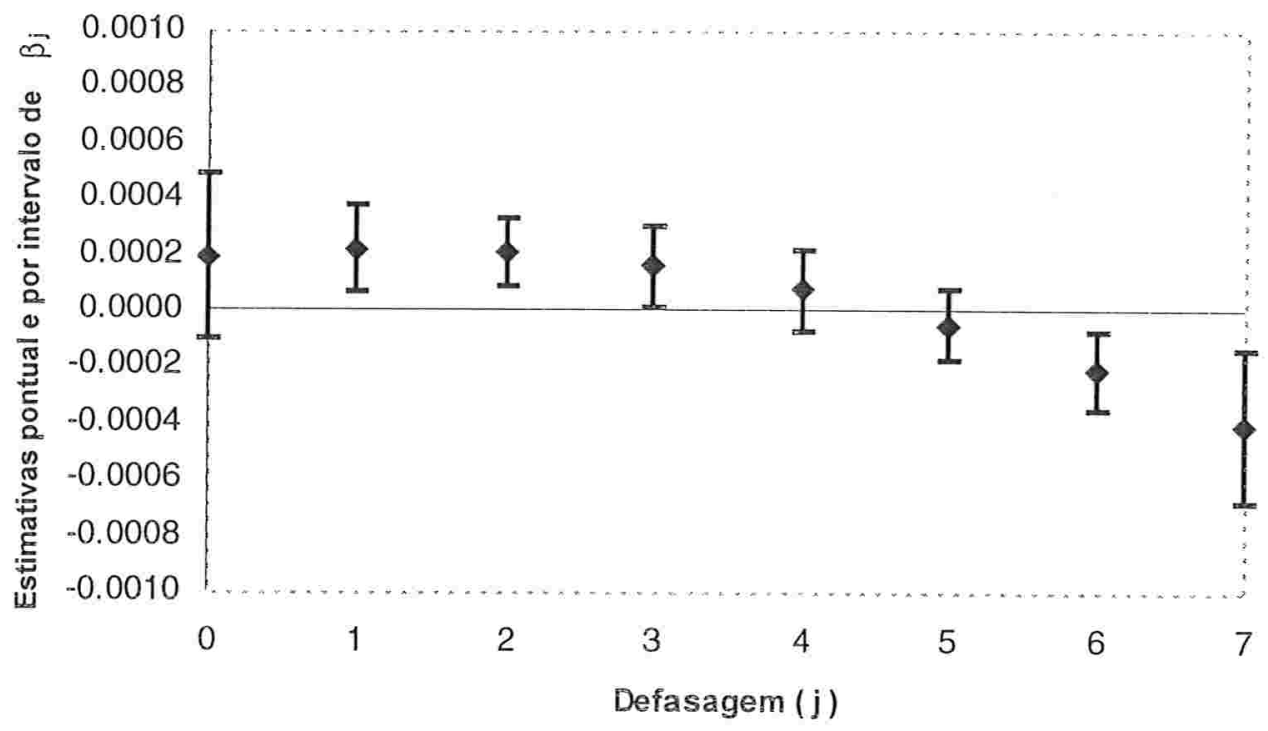

Figura A.2: Estimativas pontual e por intervalo $(\gamma=0,90)$ dos coeficientes do $\mathrm{NO}_{2}$ considerando defasagens de até 7 dias em relação à ocorrência da natimortalidade para o modelo restrito $(\mathrm{d}=2)$. 
Tabela A.2: Estimativas do coeficiente do poluente e erros padrão considerando concentrações do $\mathrm{NO}_{2}$ defasadas de até 6 dias em relação ao evento de natimortalidade e valores observados do AIC nos modelos irrestrito e restrito $(\mathrm{d}=2)$.

\begin{tabular}{|c|c|c|c|}
\hline Parâmetro & Restrição & Estimativa & Erro Padrão \\
\hline \multirow{2}{*}{$\beta_{0}$} & Não & 0,000227 & 0,000256 \\
\hline & Sim & 0,000183 & 0,000195 \\
\hline \multirow{2}{*}{$\beta_{1}$} & Não & 0,000183 & 0,000269 \\
\hline & Sim & 0,000223 & 0,000094 \\
\hline \multirow{2}{*}{$\beta_{2}$} & Não & 0,000181 & 0,000265 \\
\hline & Sim & 0,000213 & 0,000091 \\
\hline \multirow{2}{*}{$\beta_{3}$} & Não & 0,000202 & 0,000275 \\
\hline & Sim & 0,000153 & 0,000105 \\
\hline \multirow{2}{*}{$\beta_{4}$} & Não & $-0,000038$ & 0,000276 \\
\hline & Sim & 0,000043 & 0,000091 \\
\hline \multirow{2}{*}{$\beta_{5}$} & Não & 0,000028 & 0,000277 \\
\hline & Sim & $-0,000118$ & 0,000086 \\
\hline \multirow{2}{*}{$\beta_{6}$} & Não & $-0,000421$ & 0,000251 \\
\hline & Sim & $-0,000328$ & 0,000178 \\
\hline \multirow{2}{*}{$\beta^{*}=\sum_{j=0}^{6} \beta_{j}$} & Não & 0,000362 & 0,000358 \\
\hline & Sim & 0,000370 & 0,000358 \\
\hline \multicolumn{4}{|c|}{ AIC } \\
\hline Modelo Irrestrito: & 521,463 & Modelo Restrito: & 513,295 \\
\hline
\end{tabular}




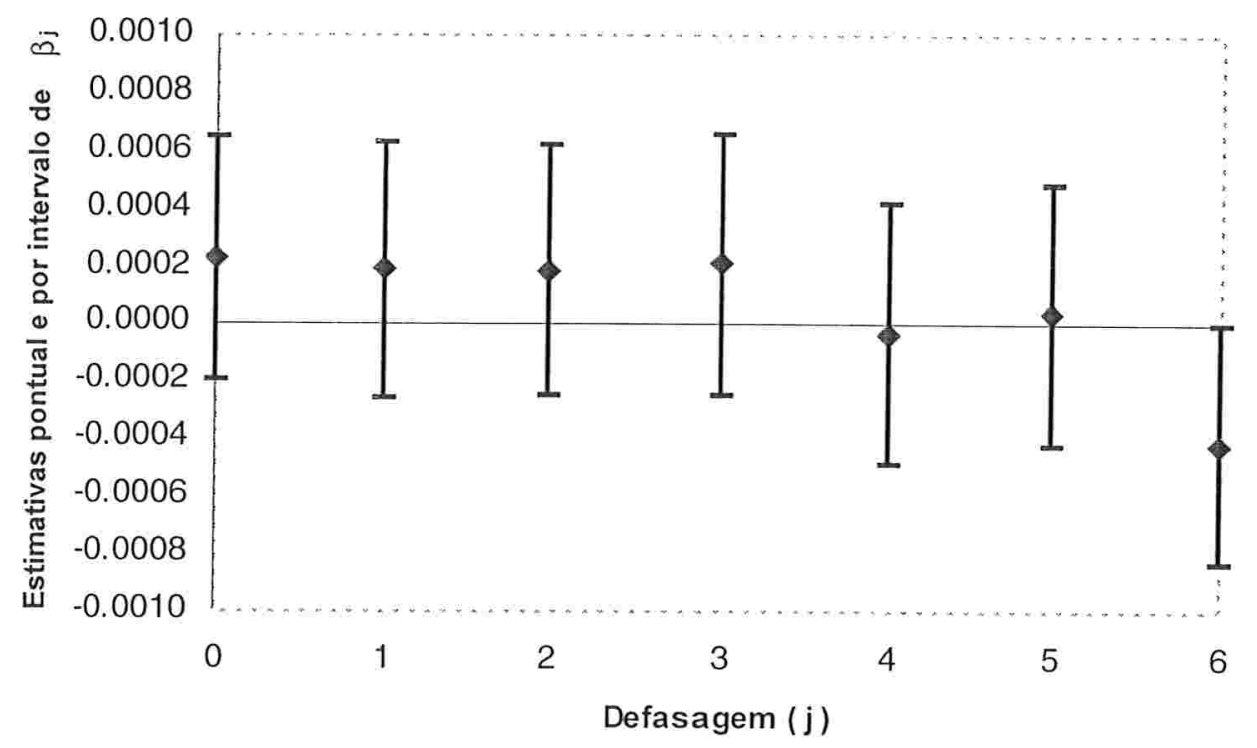

Figura A.3: Estimativas pontual e por intervalo $(\gamma=0,90)$ dos coeficientes do $\mathrm{NO}_{2}$ considerando defasagens de até 6 dias em relação à ocorrência da natimortalidade para o modelo irrestrito.

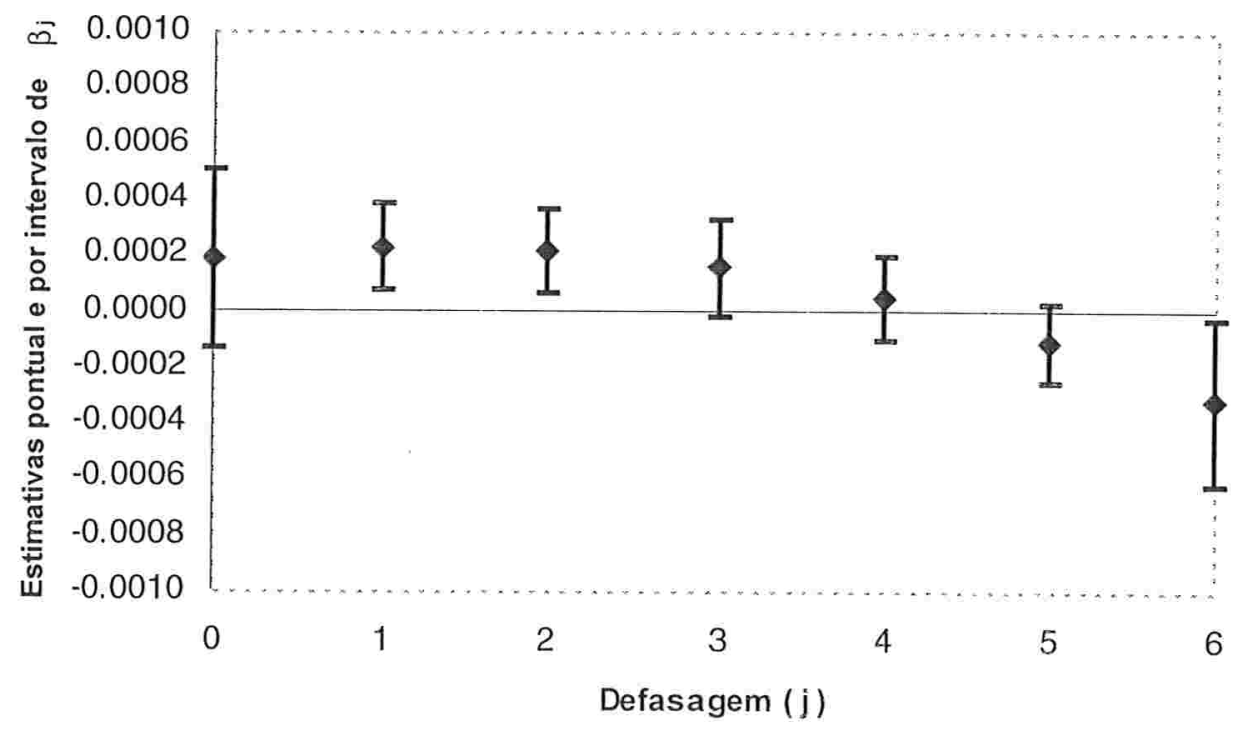

Figura A.4: Estimativas pontual e por intervalo $(\gamma=0,90)$ dos coeficientes do $\mathrm{NO}_{2}$ considerando defasagens de até 6 dias em relação à ocorrência da natimortalidade para o modelo restrito $(d=2)$. 
Tabela A.3: Estimativas do coeficiente do poluente e erros padrão considerando concentrações de $\mathrm{NO}_{2}$ defasadas de até 5 dias em relação ao evento de natimortalidade e valores observados do AIC nos modelos irrestrito e restrito $(\mathrm{d}=2)$.

\begin{tabular}{cccc}
\hline Parâmetro & Restrição & Estimativa & Erro Padrão \\
\hline \multirow{2}{*}{$\beta_{0}$} & Não & 0,000234 & 0,000255 \\
& Sim & 0,000204 & 0,000208 \\
\hline \multirow{2}{*}{$\beta_{1}$} & Não & 0,000178 & 0,000268 \\
& Sim & 0,000222 & 0,000099 \\
\hline \multirow{2}{*}{$\beta_{2}$} & Não & 0,000184 & 0,000264 \\
& Sim & 0,000194 & 0,000115 \\
\hline \multirow{2}{*}{$\beta_{3}$} & Não & 0,000166 & 0,000273 \\
& Sim & 0,000118 & 0,000115 \\
\hline \multirow{2}{*}{$\beta_{4}$} & Não & $-0,000029$ & 0,000274 \\
\multirow{2}{*}{$\beta_{5}$} & Sim & $-0,000005$ & 0,000091 \\
\hline \multirow{2}{*}{$\beta^{*}=\sum_{\mathrm{j}=0}^{5} \beta_{\mathrm{j}}$} & Não & $-0,000174$ & 0,000249 \\
& Sim & $-0,000175$ & 0,000191 \\
\hline \multicolumn{5}{c}{ Não } & 0,000559 & 0,000341 \\
Modelo Irrestrito: & Sim & 0,000558 & 0,000340 \\
\hline
\end{tabular}




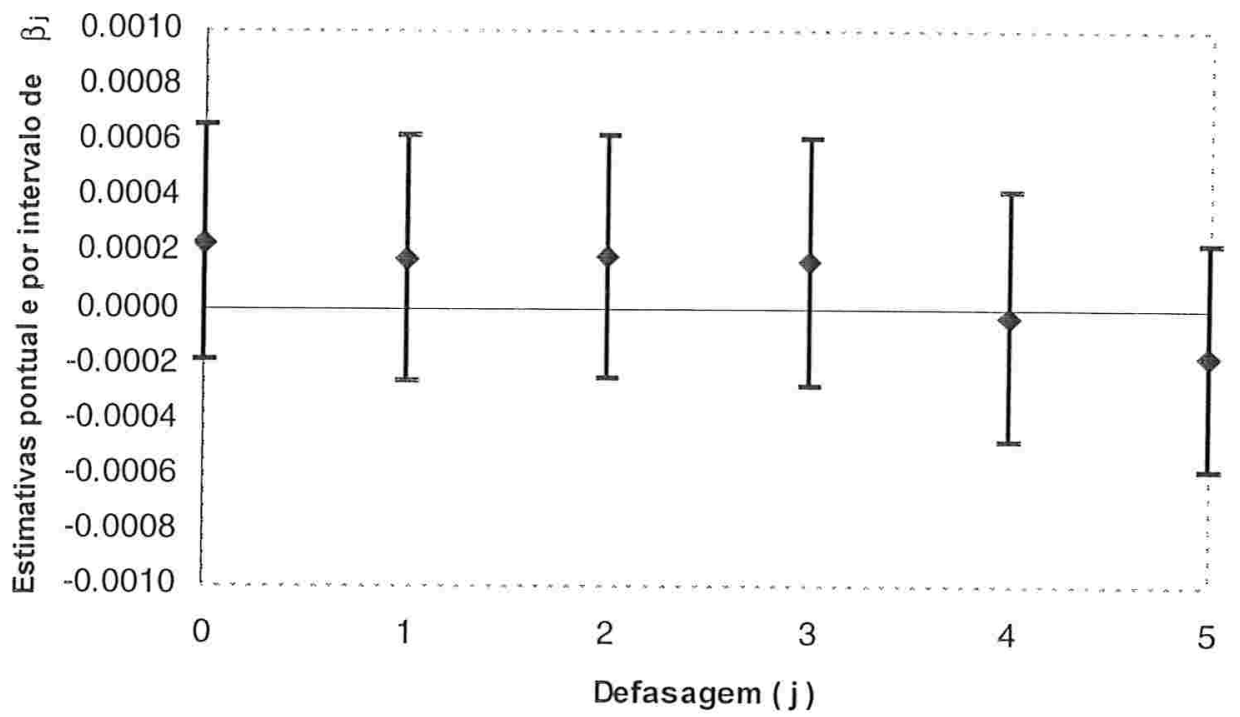

Figura A.5: Estimativas pontual e por intervalo $(\gamma=0,90)$ dos coeficientes do $\mathrm{NO}_{2}$ considerando defasagens de até 5 dias em relação à ocorrência da natimortalidade para o modelo irrestrito.

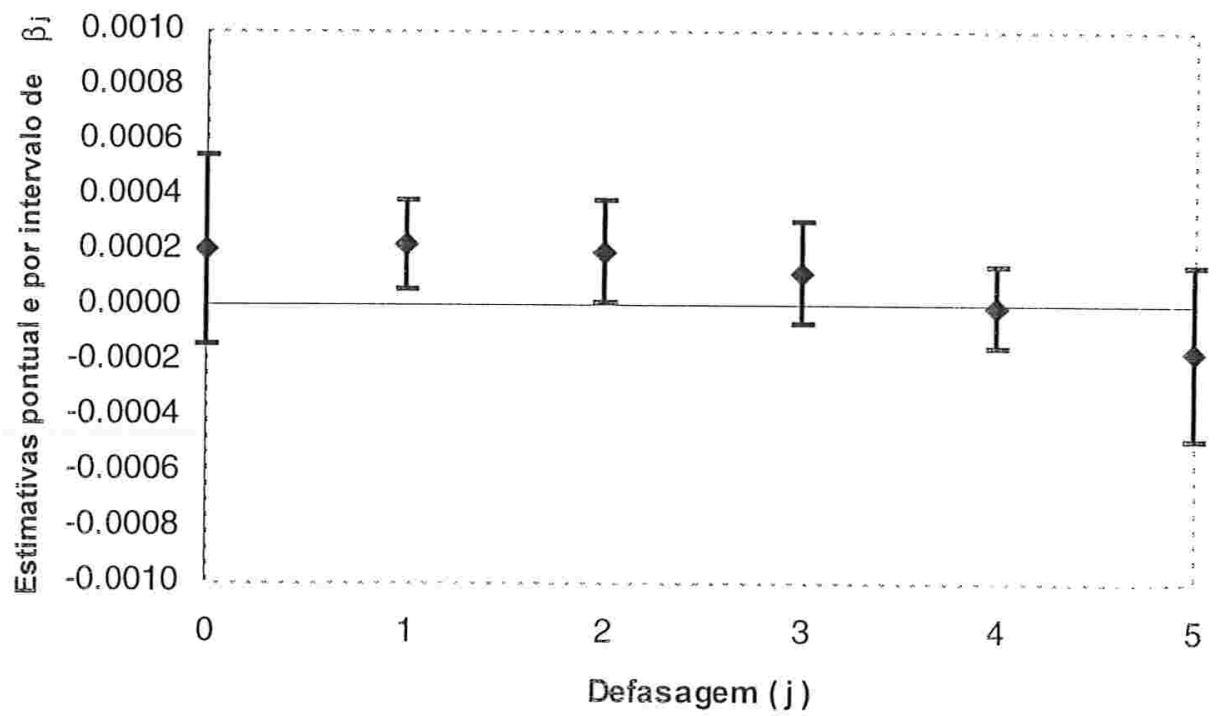

Figura A.6: Estimativas pontual e por intervalo $(\gamma=0,90)$ dos coeficientes do $\mathrm{NO}_{2}$ considerando defasagens de até 5 dias em relação à ocorrência da natimortalidade para o modelo restrito $(d=2)$. 
Tabela A.4: Estimativas do coeficiente do poluente e erros padrão considerando concentrações do $\mathrm{NO}_{2}$ defasadas de até 3 dias em relação ao evento de natimortalidade e valores observados do AIC nos modelos irrestrito e restrito $(\mathrm{d}=2)$.

\begin{tabular}{cccc}
\hline Parâmetro & Restrição & Estimativa & Erro Padrão \\
\hline \multirow{2}{*}{$\beta_{0}$} & Não & 0,000229 & 0,000255 \\
& Sim & 0,000215 & 0,000201 \\
\hline \multirow{2}{*}{$\beta_{1}$} & Não & 0,000164 & 0,000266 \\
& Sim & 0,000166 & 0,000140 \\
\hline \multirow{2}{*}{$\beta_{2}$} & Não & 0,000172 & 0,000263 \\
& Sim & 0,000115 & 0,000138 \\
\hline \multirow{2}{*}{$\beta_{3}$} & Não & 0,000115 & 0,000246 \\
$\beta^{*}=\sum_{\mathrm{j}=0}^{3} \beta_{j}$ & Sim & 0,000062 & 0,000198 \\
\hline \multirow{2}{*}{ Modelo Irrestrito: } & Não & 0,000680 & 0,000311 \\
\hline & Sim & 0,000556 & 0,000268 \\
\hline
\end{tabular}




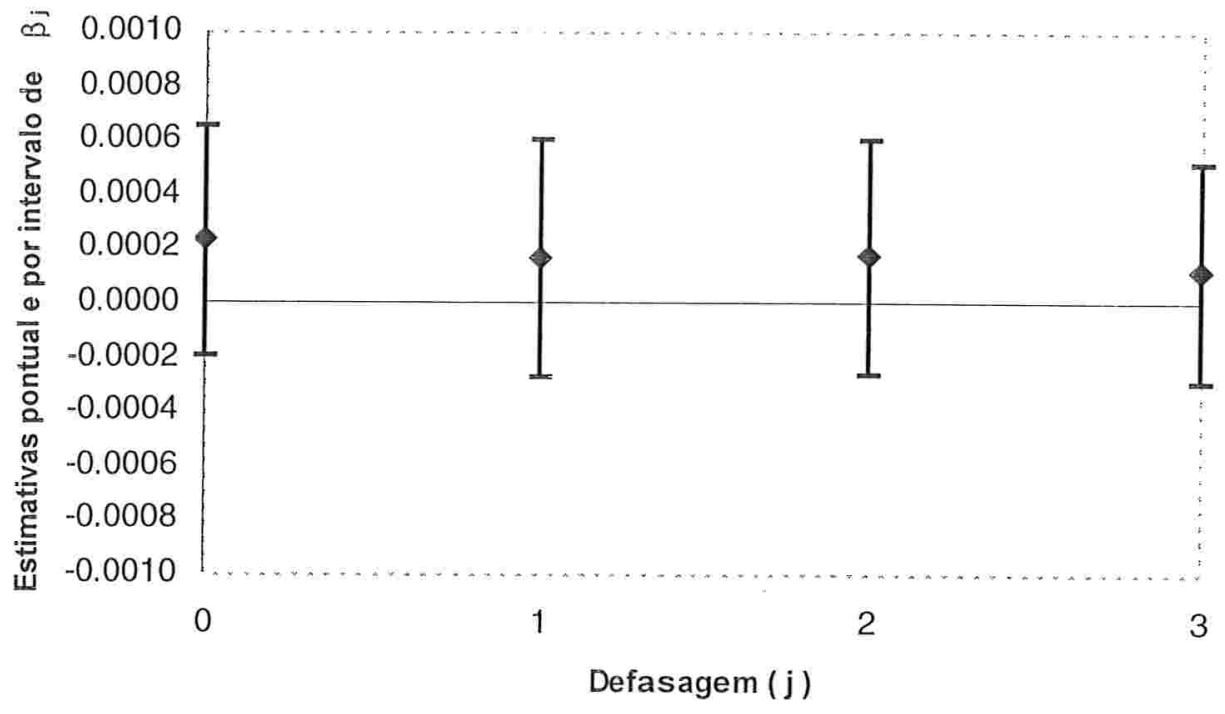

Figura A.7: Estimativas pontual e por intervalo $(\gamma=0,90)$ dos coeficientes do $\mathrm{NO}_{2}$ considerando defasagens de até 3 dias em relação à ocorrência da natimortalidade para o modelo irrestrito

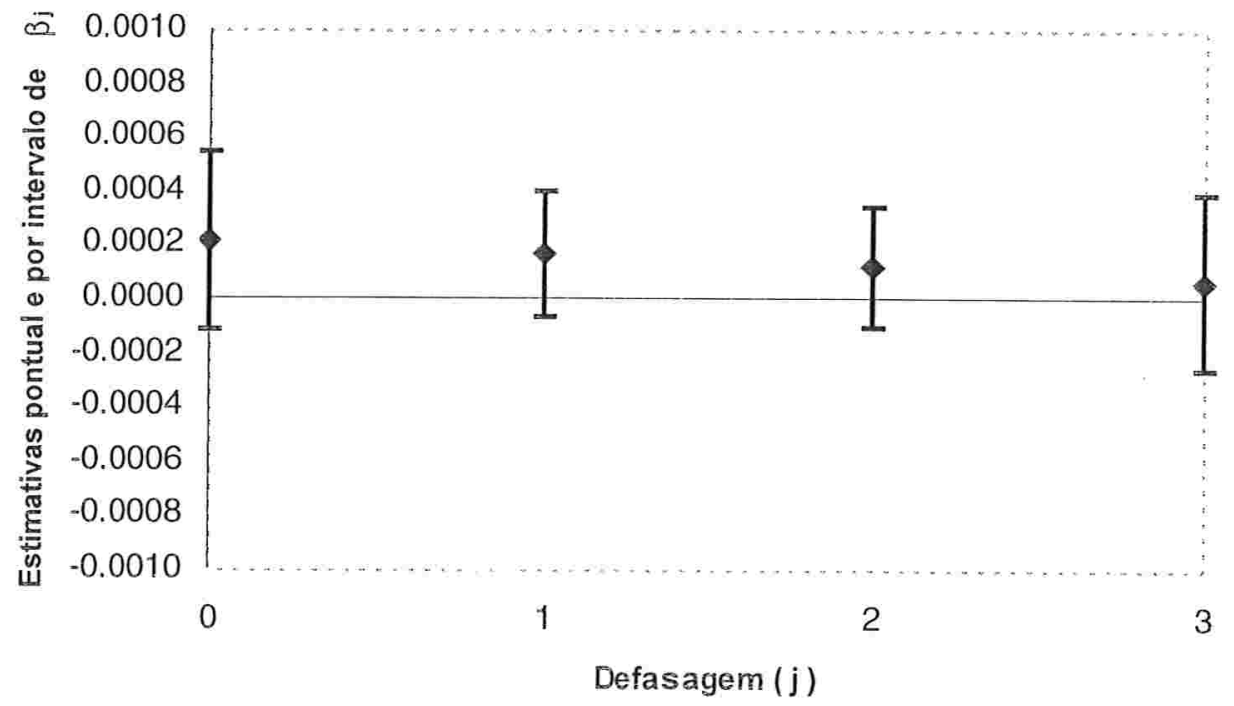

Figura A.8: Estimativas pontual e por intervalo $(\gamma=0,90)$ dos coeficientes do $\mathrm{NO}_{2}$ considerando defasagens de até 3 dias em relação à ocorrência da natimortalidade para o modelo restrito $(\mathrm{d}=2)$. 
Tabela B.1: Estimativas dos parâmetros do modelo (4.9) e erros padrão estimados.

\begin{tabular}{cccc}
\hline Termo & Parâmetro & Estimativa & Erro padrão es \\
\hline intercepto & $\alpha$ & 2,109 & 0,369 \\
\hline Dias da Semana & & & \\
& & & \\
domingo & $\Psi_{1}$ & 0,157 & 0,0520 \\
segunda-feira & $\Psi_{2}$ & 0,245 & 0,0509 \\
terça-feira & $\Psi_{3}$ & 0,256 & 0,0510 \\
quarta-feira & $\Psi_{4}$ & 0,250 & 0,0511 \\
quinta-feira & $\Psi_{5}$ & 0,226 & 0,0514 \\
sexta-feira & $\Psi_{6}$ & 0,146 & 0,0523 \\
sábado & & & \\
\end{tabular}

Variáveis Climáticas

$\begin{array}{cccc}\text { umidade } & \Psi_{7} & 0,00218 & 0,00124 \\ \text { temperatura } & \Psi_{8} & -0,00693 & 0,00773\end{array}$

Poluente NO2

Defasagem

\begin{tabular}{llll}
0 & $\beta_{0}$ & 0.000201 & 0.000079 \\
1 & $\beta_{1}$ & 0.000155 & 0.000063 \\
2 & $\beta_{2}$ & 0.000114 & 0.000050 \\
3 & $\beta_{3}$ & 0.000078 & 0.000041 \\
4 & $\beta_{4}$ & 0.000046 & 0.000035 \\
5 & $\beta_{5}$ & 0.000019 & 0.000032 \\
6 & $\beta_{6}$ & -0.000003 & 0.000031 \\
7 & $\beta_{7}$ & -0.000021 & 0.000031 \\
8 & $\beta_{8}$ & -0.000034 & 0.000032 \\
9 & $\beta_{9}$ & -0.000043 & 0.000032 \\
10 & $\beta_{10}$ & -0.000048 & 0.000031 \\
11 & $\beta_{11}$ & -0.000049 & 0.000031 \\
12 & $\beta_{12}$ & -0.000048 & 0.000030 \\
13 & $\beta_{13}$ & -0.000045 & 0.000029 \\
14 & $\beta_{14}$ & -0.000040 & 0.000029 \\
15 & $\beta_{15}$ & -0.000033 & 0.000028 \\
16 & $\beta_{16}$ & -0.000025 & 0.000029 \\
\hline
\end{tabular}


Tabela B.1: Continuação

\begin{tabular}{cccc}
\hline Termo & Parâmetro & Estimativa & Erro Padrão \\
\hline 17 & $\beta_{17}$ & -0.000017 & 0.000029 \\
18 & $\beta_{18}$ & -0.000009 & 0.000030 \\
19 & $\beta_{19}$ & -0.000002 & 0.000031 \\
20 & $\beta_{20}$ & 0.000005 & 0.000031 \\
21 & $\beta_{21}$ & 0.000010 & 0.000032 \\
22 & $\beta_{22}$ & 0.000012 & 0.000032 \\
23 & $\beta_{23}$ & 0.000012 & 0.000031 \\
24 & $\beta_{24}$ & 0.000008 & 0.000031 \\
25 & $\beta_{25}$ & 0.000001 & 0.000031 \\
26 & $\beta_{26}$ & -0.000009 & 0.000034 \\
27 & $\beta_{27}$ & -0.000023 & 0.000039 \\
28 & $\beta_{28}$ & -0.000040 & 0.000048 \\
29 & $\beta_{29}$ & -0.000061 & 0.000060 \\
30 & $\beta_{30}$ & -0.000086 & 0.000075 \\
& & & \\
\hline
\end{tabular}

Desvio residual: 709,2 com 678,5 graus de liberdade 


\section{Apêndice C}

\section{Comandos do S-plus para obtenção dos resultados do modelo (4.9)}

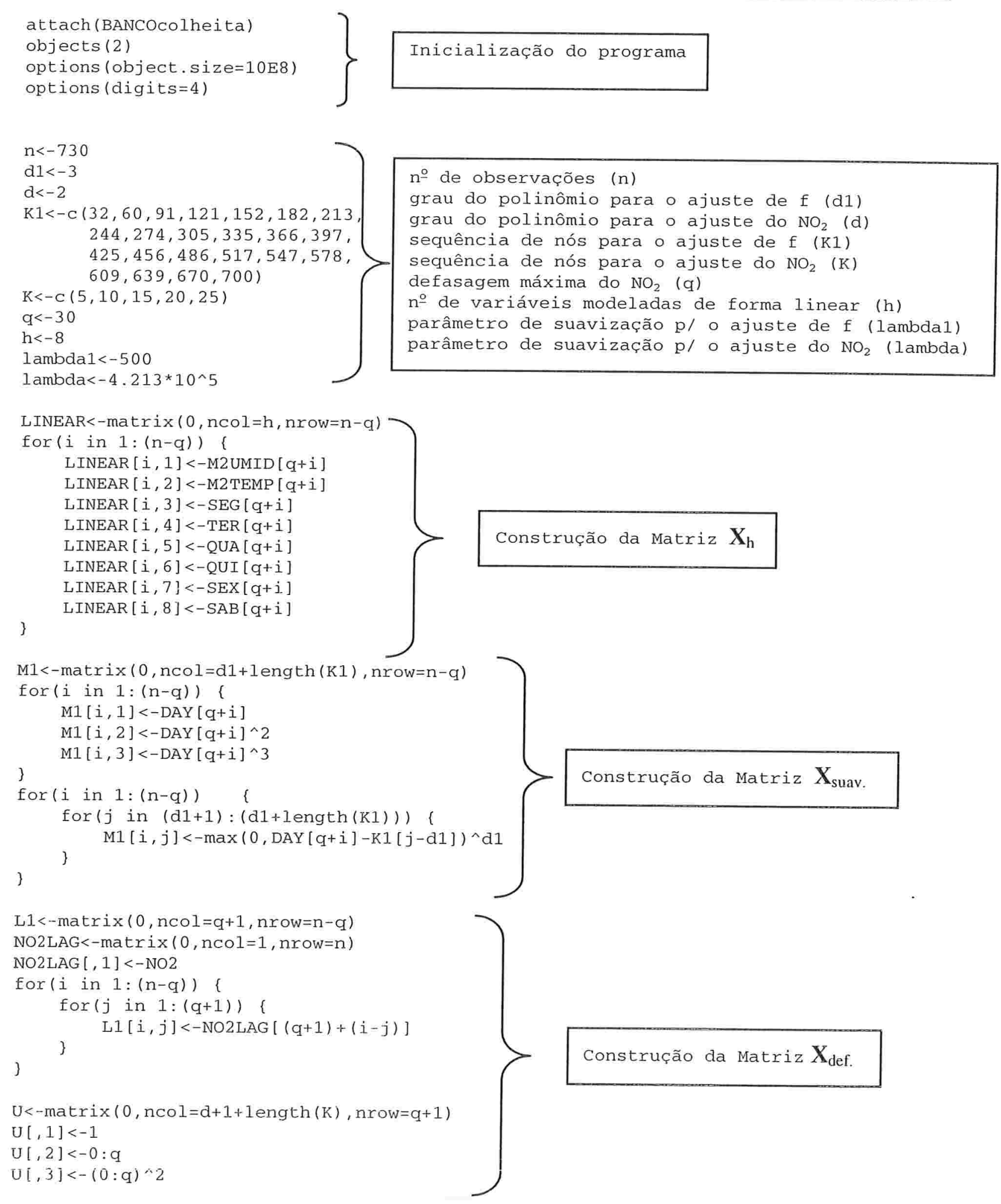




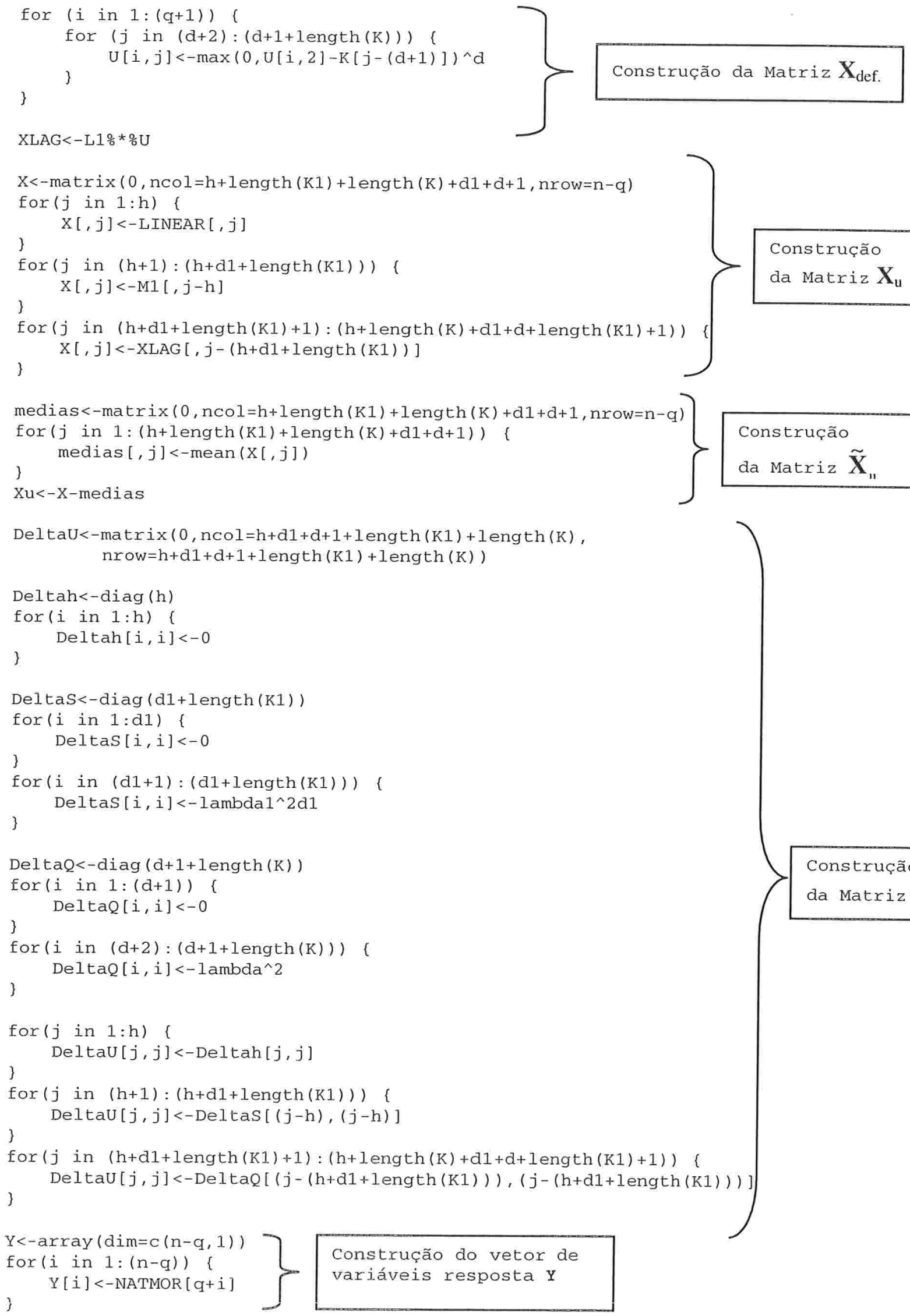

Construção da Matriz $\mathbf{X}_{\mathbf{u}}$ 


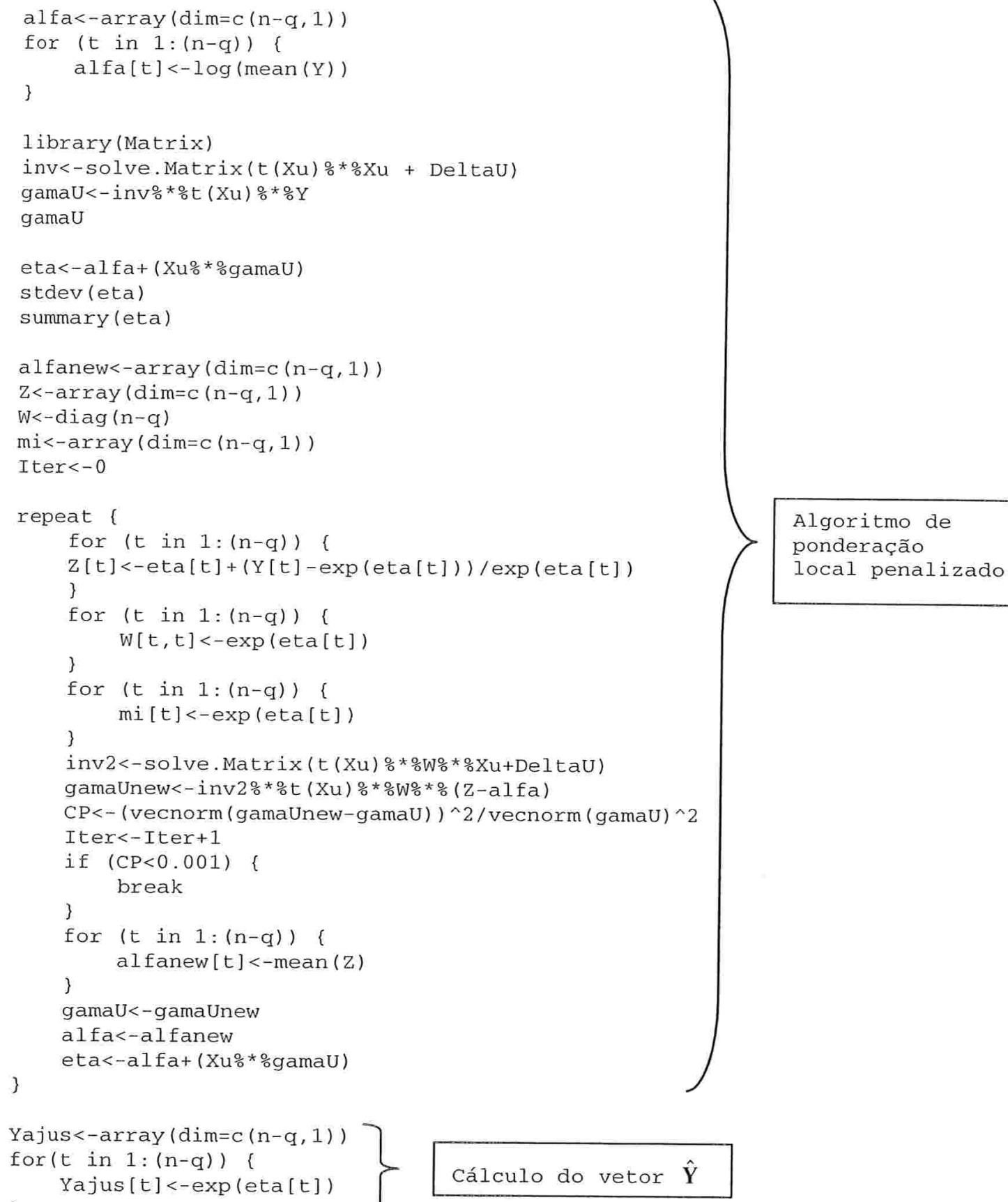

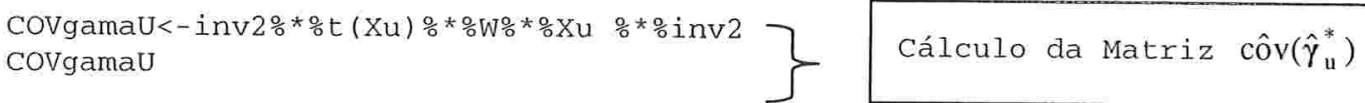

COVgamaUlag $<-$ matrix $(0, \operatorname{ncol}=\mathrm{d}+$ length $(K)+1$, nrow $=\mathrm{d} 2+$ length $(K)+1)$

for $(i$ in $1:(d+$ length $(K)+1))$ (

for $(j$ in $1:(d+$ length $(K)+1)) \quad\{$

CoVgamaUlag $[i, j]<-\operatorname{CovgamaU}[i+h+d 1+$ length (K1) $j+h+d 1+1$ ength (K1) ] \}

Covgamaulag

Cálculo da Matriz $\operatorname{côv}\left(\hat{\gamma}_{\mathrm{u}}^{*}\right)_{\text {def. }}$ 

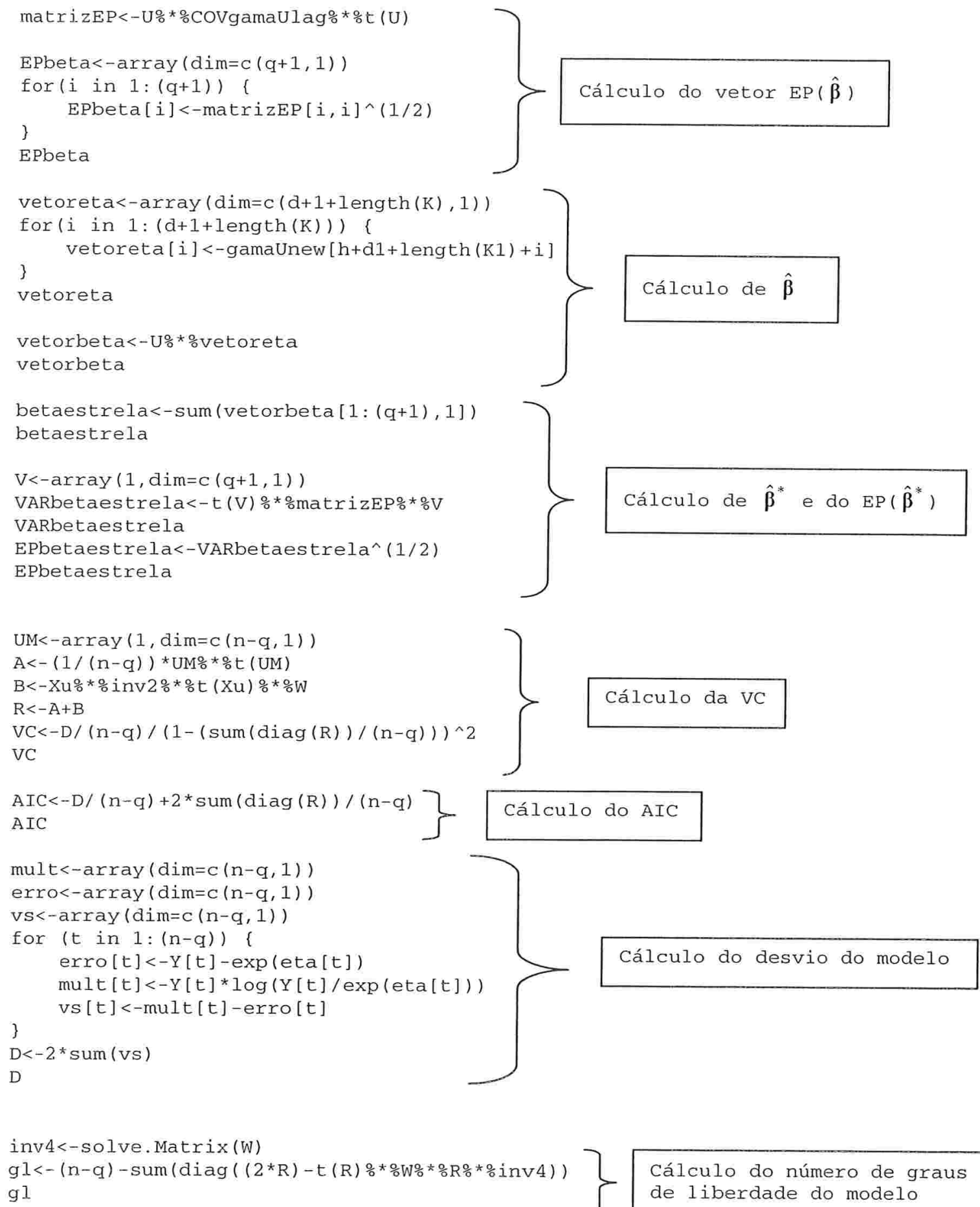


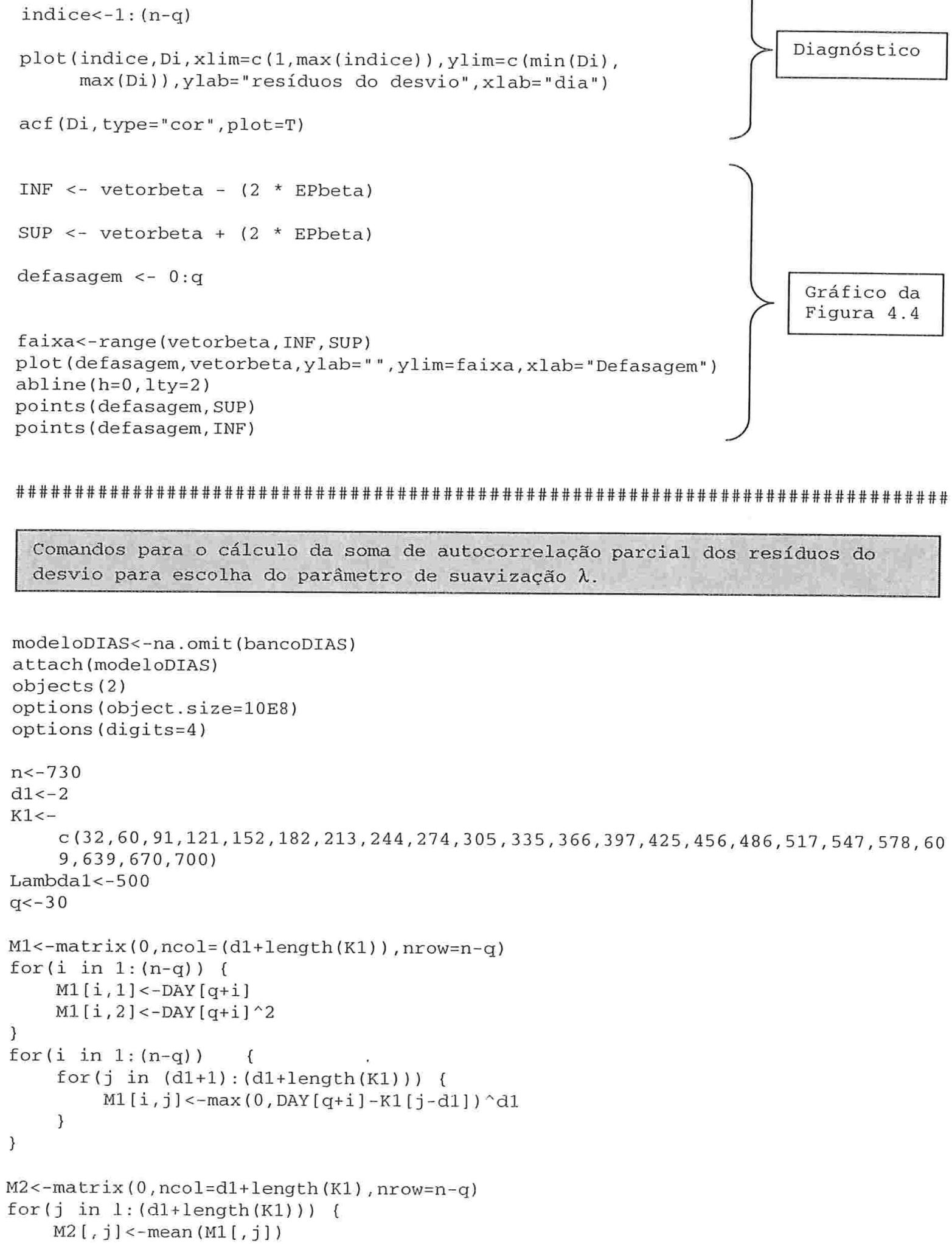




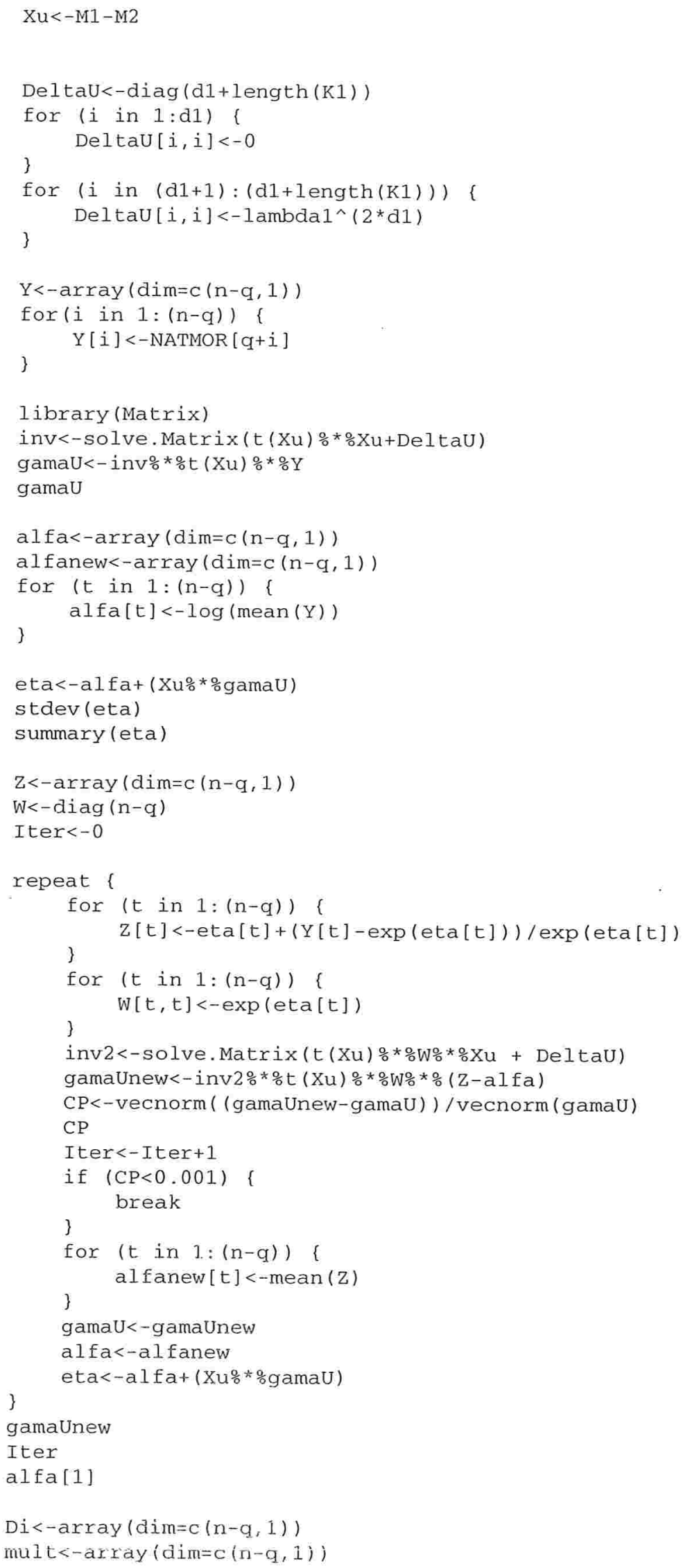


$\operatorname{var} A B C<-t$ (vetor $1 \mathrm{ABC}) \%$ *matrizvar $\mathrm{ABC} \%$ * $\mathrm{vetor} 1 \mathrm{ABC}$

$\operatorname{var} A B C$

$\operatorname{epABC}<-\operatorname{varABC} \wedge(1 / 2)$

epABC

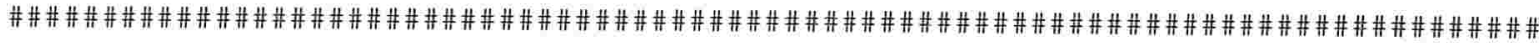


Almon, S. (1965). The Distributed Lag between Capital Appropriation and Expenditures. Econometrica, 33 (1), 178-196.

Braga, A. L. F., Zanobetti, A. e Schwartz,. J. (2001). The Lag Structure between Particulate Air Pollution and Respiratory and Cardiovascular Deaths in 10 US Cities. Journal of Occupational and Environmental Medicine, 43 (11), 927-933.

Braga, A. L. F., Saldiva, P. H. N., Pereira, L. A. A., Menezes, J. J. C., Conceição, G. M. S., Lin, C. A., Zanobetti, A., Schwartz, J. e Dockery, D. W. (2001). Health Effects of Air Pollution Exposure on Children and Adolescents in São Paulo, Brazil. Pediatric Pulmonology, 31, 106-113.

Braga, A. L. F., Conceição, G., M., S., Pereira, L. A. A., Kishi, H. S., Pereira, J. C. R., Andrade, M. F., Gonçalves, F. L. T., Saldiva, P. H. N. e Latorre, M. R. D. O. (1999). Air Pollution and Pediatric Respiratory Hospital Admissions in São Paulo, Brazil. Journal of Occupational and Environmental Medicine, 1, 95-102. 
Chambers, J. M. e Hastie, T. J. (1993). Statistical Models in S. Chapman and Hall, London.

Cleveland, W. S. (1979). Robust Locally Weighted Regression and Smoothing Scarterplots. Journal of the American Statistical Association, 74 (368), 829-836.

David, J. S. E. (2003). Modelos Aditivos Binomiais Negativos. Dissertação de Mestrado: Instituto de Matemática e Estatística da Universidade de São Paulo.

Davidson, R. e MacKinnon, J. G. (1993). Estimation and Inference in Econometrics. Oxford University Press, New York.

De Boor, C. (1978). A Pratical Guide to Splines. Springer-Verlag, New York.

Eilers, P. H. C. e Marx, B. D. (1996). Flexible Smoothing with B-splines and Penalties. Statistical Science, 11 (2), 89-121.

Einbeck, J. (no prelo). Local Fitting with General Basis Functions. Relatório Técnico da Ludwig-Maximilians-Universität, Munique, Alemanha. 
Einbeck, J., André, C. D. S. e Singer, J. M. (no prelo). Local Smoothing with Robustness against Outlying Predictors. Relatório Técnico da Ludwig-MaximiliansUniversitāt, Munique, Alemanha.

Eubank, R. L. (1988). Spline Smoothing and Nonparametric Regression. M. Dekker, New York.

Golub, G. H. e Van Loan, C. F. (1983). Matrix Computations. Johns Hopkins University Press, Baltimore, MD.

Graybill, F. A. (1983). Matrices with applications in Statistics. 2ed, Wadsworth International Group, Belmont.

Green, P. J. (1987). Penalized Likelihood for General Semi-Parametric Regression Models. International Statistical Review, 55 (3), 245-259.

Hastie, T. J. e Tibshirani, R. J. (1990). Generalized Additive Models. Chapman \& Hall, London.

Hastie, T. (1996). Pseudosplines. Journal Royal Statistical Society, 58-B (2), 379-396. 
Hazzan, S. (1978). Modelos de Defasagens Distribuídas. Dissertação de Mestrado: Instituto de Matemática e Estatística da Universidade de São Paulo.

Lima, L. P., (2001). Modelos Aditivos Generalizados:Aplicação a um Estudo Epidemiológico Ambiental. Dissertação de Mestrado: Instituto de Matemática e Estatística da Universidade de São Paulo.

Lima, L. P., André C. D. S. e Singer J. M. (2001). Modelos Aditivos Generalizados: Metodologia e Prática. Revista Brasileira de Estatística, 62 (217), 37 69.

Marx, B. D. e Eilers, P. H. C. (1998). Direct Generalized Additive Modeling with Penalized Likelihood. Computacional Statistics and Data Analysis, 28, 193-209.

McCullagh., P. e Nelder, J. (1989). Generalized Linear Models. Chapman and Hall, New York.

Montgomery, D. C. e Peck, E. A. (1992). Introduction to Linear Regression Analysis. Wiley, New york.

Neter, J., Kutner, M. H., Nachtsheim, C. J. e Wasserman, W. (1996). Applied Linear Statistical Models. 4 ed, MCGraw-Hill, Chicago. 
Paula, G. A. (2002). Modelos de Regressão. IME-USP Disponível em http://www.ime.usp.br/ giapaula/Book.pdf (30/04/2003).

Pereira, L. A. A., Loomis, D., Conceição, G. M. S., Braga, A. L. F., Arcas, R. M., Kishi, H. S., Singer, J. M., Böhm, G. M. e Saldiva, P. H. N. (1998). Association between Air Pollution and Intrauterine Mortality in São Paulo, Brazil. Environmental Health Perspective, 106 (6), 325-329.

Pope, C. A. e Schwartz, J. (1996). Time Series for the Analysis of Pulmonary Health Data. American Journal of Respiratory and Critical Care Medicine, 154, S229-S233.

Ruppert, D. (2002). Selecting the number of knots for penalized splines. Journal of Computacional and Graphical Statistics, 11, 735-757.

Schwartz, J. (2000-a). The Distributed Lag between Air Pollution and Daily Deaths. Epidemiology 11(3), 320-326.

Schwartz, J. (2000-b). Harvesting and Long-term Exposure Effects in the Relationship between Air Pollution and Mortality. American Journal of Epidemiology, 151, 440448. 
Schwartz, J. (1994). Nonparametric Smoothing in the Analysis of Air Pollution and Respiratory Illness. The Canadian Journal of Statistics, 22 (4), 471-487.

Singer, J. M., André, C. D. S., Lima, L. P. e Conceição, G. M. S. (2002). Atmospheric Pollution and Mortality in São Paulo. Em: Statistical Data Analysis Based on the $\mathrm{L}_{1}$ Norm and Related Methods (Statistics for Industry and Technology), Y. Dodge (Editor), 439-450. Basel: Birkhäuser.

Venables, W. N. e Ripley, B. D. (1997). Modern Applied Statistics with S-plus. Springer-Verlag, New York.

Zanobetti, A., Wand M. P., Schwartz J. e Ryan L. M. (2000). Generalized Additive Distributed Lag Models: Quantifying Mortality Displacement. Biostatistics 1 (3), 279292. 